\title{
Acylation of Arenes with Aldehydes through Dual C-H Activations by Merging Photocatalysis and Palladium Catalysis
}

Haiyang Wang, ${ }^{\dagger}$ Tao Li, ${ }^{\dagger}$ Dongyan Hu, Xiaogang Tong, Liyan Zheng, and Chengfeng Xia*

Key Laboratory of Medicinal Chemistry for Natural Resource, Ministry of Education, Yunnan Provincial Center for Research \& Development of Natural Products, School of Chemical Science and Technology, Yunnan University, Kunming 650091, China.

\section{Table of Contents}

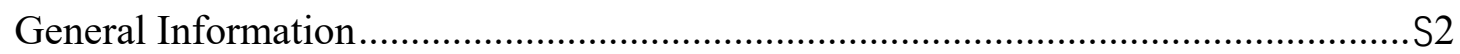

General Procedures for Acylation of Arenes with Aldehydes .................................S2

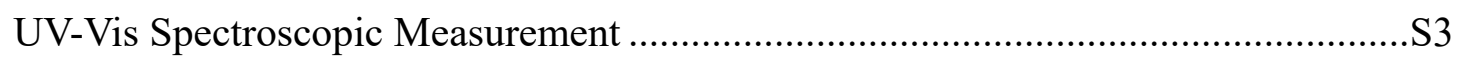

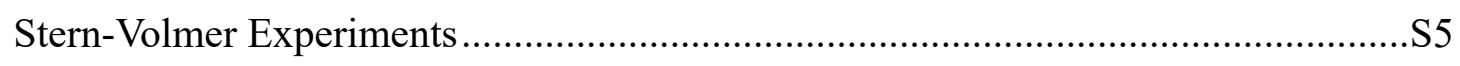

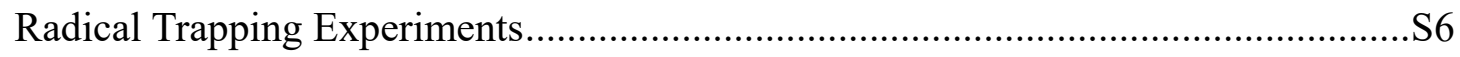

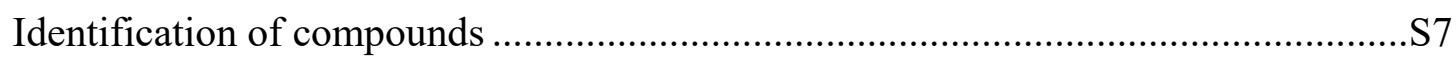

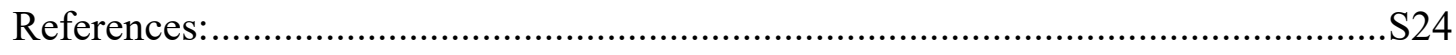

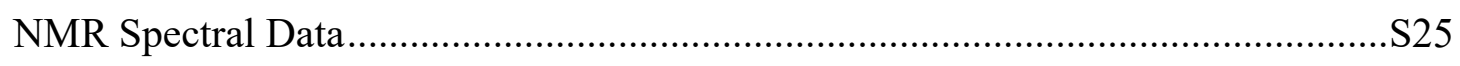




\section{General Information}

All reactions were performed under argon atmosphere using flame-dried glassware unless otherwise noted. $\mathrm{CH}_{3} \mathrm{CN}$ was distilled over $\mathrm{CaH}_{2}$ and rigorously degassed by freeze/pump/thaw. All reagents were commercially available and used without further purification unless indicated otherwise. Blue LED lamps (Zhongshan Qinhui Lighting Co., Ltd, China, 18 W, Product model: QH-6200) were used to irradiate the reaction mixtures. Thin layer chromatographies were carried out on GF254 plates. Flash chromatography was performed with 200-300 mesh silica gels. Visualization of the developed chromatogram was performed by fluorescence quenching or by ceric ammonium molybdate, or $\mathrm{KMnO}_{4}$ stain. Yields reported were for isolated, spectroscopically pure compounds.

${ }^{1} \mathrm{H}$ and ${ }^{13} \mathrm{C}$ NMR spectra were recorded on a Bruker Avance 400 and $600 \mathrm{MHz}$ spectrometer. Chemical shifts $(\delta)$ are expressed in ppm, and $J$-values are given in $\mathrm{Hz}$. The residual solvent protons $\left({ }^{1} \mathrm{H}\right)$ or the solvent carbons $\left({ }^{13} \mathrm{C}\right)$ were used as internal standards. ESIMS and HRESIMS were taken on Agilent 6540 Q-TOF spectrometer. UV-Vis measurements were carried out on a HITACHI U-4100 spectrophotometer.

\section{General Procedures for Acylation of Arenes with Aldehydes}

To an oven dried $10 \mathrm{~mL}$ round-bottom flask with a magnetic stirring bar was added phenanthraquinone (PQ) (0.02 mmol, 0.1 equiv), $\mathrm{Pd}(\mathrm{OAc})_{2}(0.04 \mathrm{mmol}, 0.2$ equiv), and $\mathrm{Ag}_{2} \mathrm{O}$ (0.4 mmol, 2 equiv). Then the reaction flask was allowed to be vacuumed and purged with Argon for three times. $\mathrm{CH}_{3} \mathrm{CN}(4 \mathrm{~mL})$, phenylpyridines $(0.2 \mathrm{mmol}, 1$ equiv), and aldehydes ( $0.5 \mathrm{mmol}, 2.5$ equiv) were carefully added under Argon. The reaction mixture was stirred under two $18 \mathrm{~W}$ blue LED lamps (the distance was about $7 \mathrm{~cm}$ ) irradiation for 12 hours. The reaction temperature was approximately $25^{\circ} \mathrm{C}$, with a fan used to assist cooling. After irradiation was stopped, the reaction mixture was extracted with ethyl acetate $(15 \mathrm{~mL} \times 3)$. The combined organic layers were washed with brine, dried over anhydrous $\mathrm{Na}_{2} \mathrm{SO}_{4}$ and concentrated. The crude product was 
subjected to column chromatography on silica gel to afford the product.

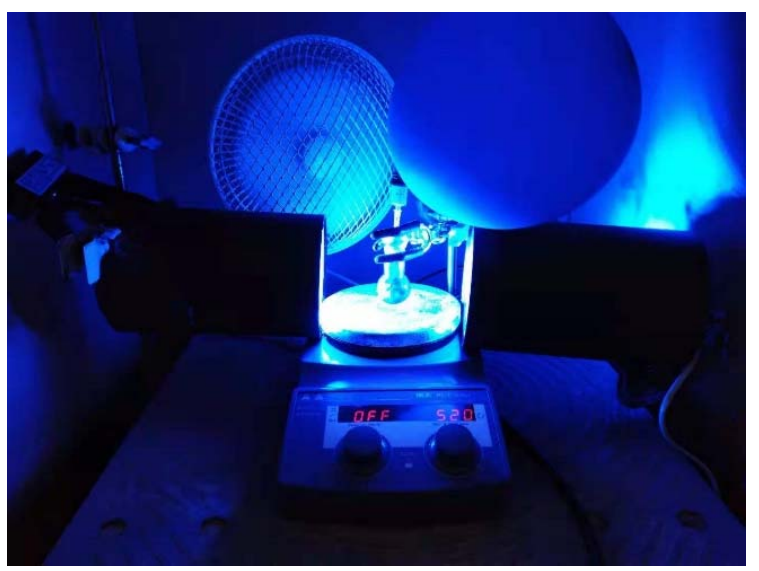

Supplementary Figure 1. Experimental setup for acylation of arenes.

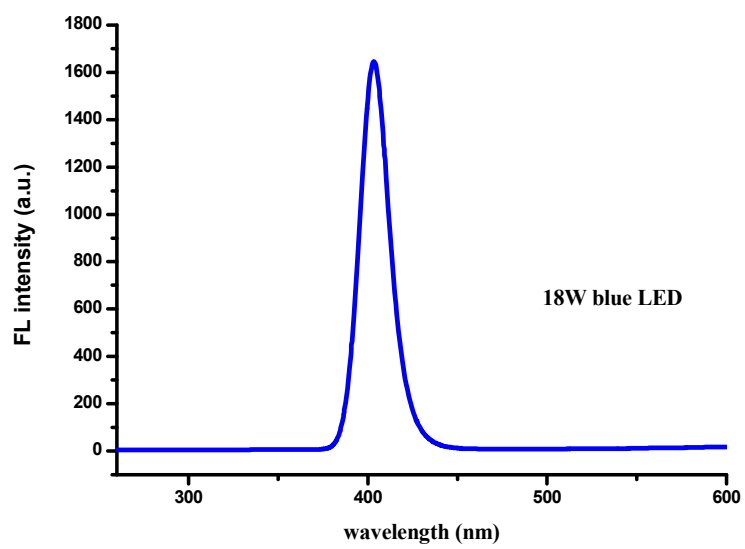

Supplementary Figure 2. Emission spectra of the 18W blue LED lamp.

\section{UV-Vis Spectroscopic Measurement}

The UV-Vis absorption spectra of acetonitrile solutions $\left(1 \times 10^{-4} \mathrm{M}\right)$ of PQ were recorded on HITACHI U-4100 UV-Visible Spectrophotometer. Obvious absorption peaks of the buff solution of PQ was observed in visible light region. 


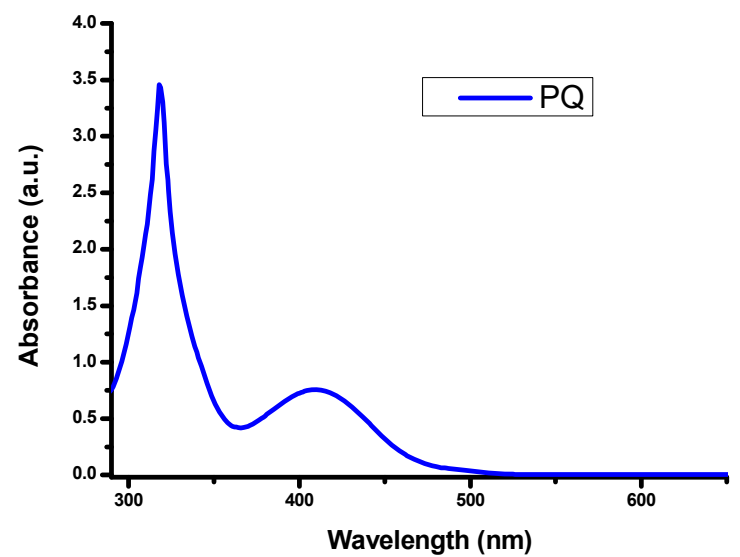

Supplementary Figure 3 . UV-Vis absorption spectra of PQ in $\mathrm{CH}_{3} \mathrm{CN}$ at concentrations of $1 \times 10^{-4} \mathrm{M}$. 


\section{Stern-Volmer Experiments}

The samples were prepared the PQ $\left(1 \times 10^{-5} \mathrm{M}\right)$ with the required amount of cumaldehydein a total volume of $1 \mathrm{~mL}$ of dry $\mathrm{CH}_{3} \mathrm{CN}$ (rigorously degassed by freeze/pump/thaw) in a $10 \times 10 \mathrm{~mm}$ light path quartz fluorescence cuvette under an argon atmosphere. The samples were vigorously bubbled with dry argon for 5 minutes prior to the measurement. The excitation wavelength was fixed at $310 \mathrm{~nm}$, the emission light was acquired from $340 \mathrm{~nm}$ to $500 \mathrm{~nm}$.

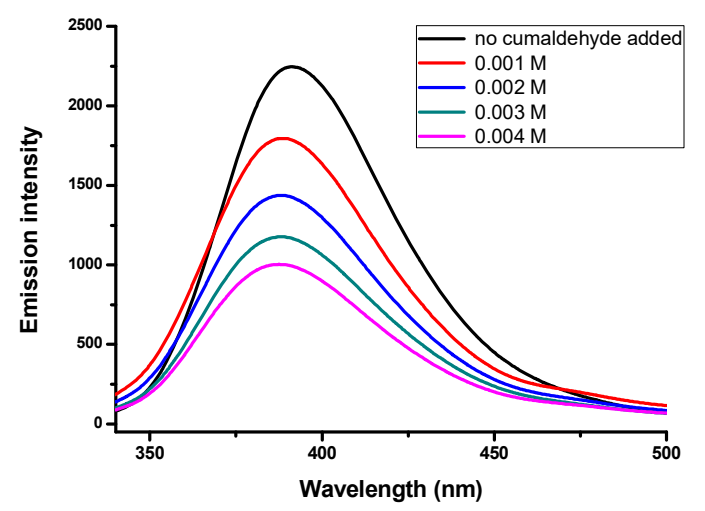

Supplementary Figure 4. Quenching of the PQ emission $\left(1 \times 10^{-5} \mathrm{M}\right.$ in $\left.\mathrm{CH}_{3} \mathrm{CN}\right)$ in the presence of increasing amounts of cumaldehyde .

The Stern-Volmer plot shows a linear correlation between the amounts of cumaldehyde and the ratio $\mathrm{I}_{0} / \mathrm{I}$.

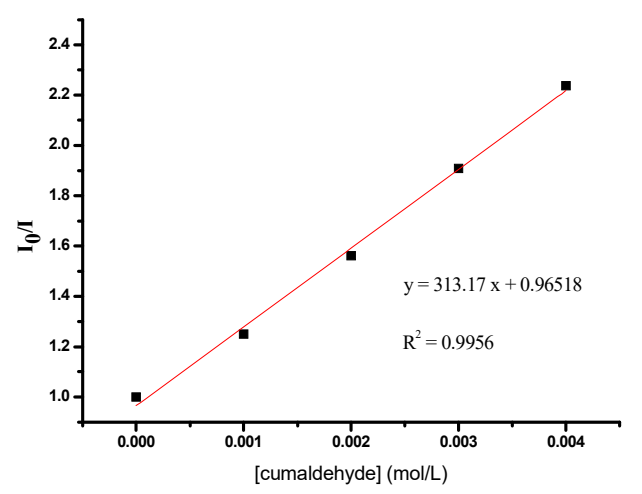

Supplementary Figure 5. Stern-Volmer quenching plot 


\section{Radical Trapping Experiments}

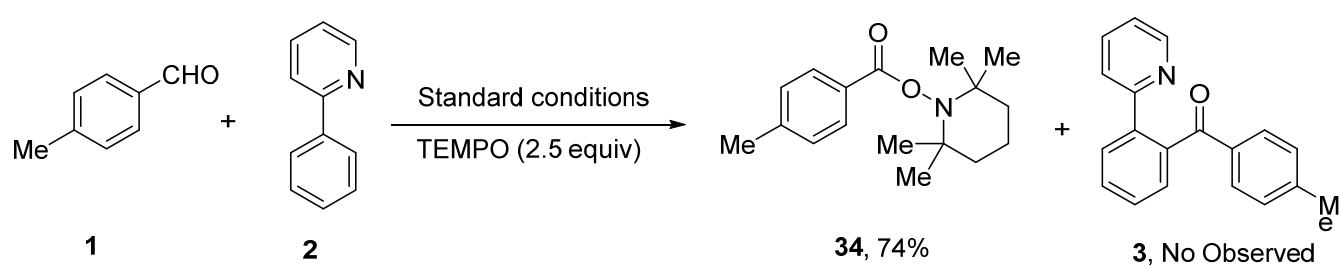

To a $10 \mathrm{~mL}$ glass vial, containing 4-methylbenzaldehyde 1 (60.0 mg, $0.5 \mathrm{mmol})$, 2Phenylpyridine 2 (31.1 mg, $0.2 \mathrm{mmol})$, PQ (4.2 mg, $0.02 \mathrm{mmol}), \mathrm{Pd}(\mathrm{OAc}) 2$ (8.9 mg, $0.04 \mathrm{mmol}$ ), $\mathrm{Ag}_{2} \mathrm{O}$ (92.4 mg, $0.4 \mathrm{mmol}$ ), and TEMPO (78.0 mg, $0.5 \mathrm{mmol}$ ), was added $\mathrm{CH}_{3} \mathrm{CN}(4 \mathrm{~mL})$. The vial was then capped under Ar (Insert an Ar ball), vortexed for approximately one minute, and then placed around $7 \mathrm{~cm}$ from two $18 \mathrm{~W}$ blue LED lamps. The reaction temperature was approximately $25^{\circ} \mathrm{C}$, with a fan used to assist cooling. After 12 hours, the reaction was quenched by water $(15 \mathrm{~mL})$, extracted with ethyl acetate $(15 \mathrm{~mL} \times 3)$. The combined organic layers were washed with brine, dried over anhydrous $\mathrm{Na}_{2} \mathrm{SO}_{4}$ and concentrated. The crude product was subjected to column chromatography on silica gel to afford the product (ethyl acetate/petroleum ether = 1/100) to give compound 34 as colorless oil (40.9 mg, 74\% yield). ${ }^{1} \mathrm{H}$ NMR (600 MHz, $\left.\mathrm{CDCl}_{3}\right) \delta 7.98(\mathrm{~d}, J=8.0 \mathrm{~Hz}, 2 \mathrm{H}), 7.27(\mathrm{~d}, J=7.7 \mathrm{~Hz}, 2 \mathrm{H}), 2.43(\mathrm{~s}, 3 \mathrm{H}), 1.84-1.75$ $(\mathrm{m}, 2 \mathrm{H}), 1.69(\mathrm{~m}, 1 \mathrm{H}), 1.59(\mathrm{~m}, 2 \mathrm{H}), 1.46(\mathrm{~m}, 1 \mathrm{H}), 1.28(\mathrm{~s}, 6 \mathrm{H}), 1.12(\mathrm{~s}, 6 \mathrm{H}) .{ }^{13} \mathrm{C} \mathrm{NMR}$ $\left(150 \mathrm{MHz}, \mathrm{CDCl}_{3}\right) \delta 166.6,143.6,129.7,129.2,127.0,60.5,39.2,32.1,21.8,21.0$, 17.1. HRMS (ESI) m/z: [M+ H] $]^{+}$Calcd for $\mathrm{C}_{17} \mathrm{H}_{26} \mathrm{NO}_{2} 276.1958$; found 276.1962. 


\section{Identification of compounds}

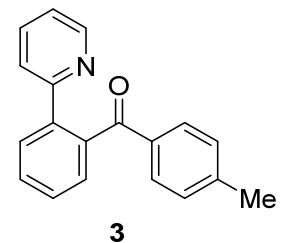

\section{(2-(Pyridin-2-yl)phenyl)(p-tolyl)methanone (3) ${ }^{1}$}

Prepared according to the general procedure using 4-methylbenzaldehyde 1 (60.0 $\mathrm{mg}$, $0.5 \mathrm{mmol}$ ), 2-phenylpyridine 2 (31.1 mg, $0.2 \mathrm{mmol})$, PQ (4.2 mg, $0.02 \mathrm{mmol}$ ), $\mathrm{Pd}(\mathrm{OAc})_{2}(8.9 \mathrm{mg}, 0.04 \mathrm{mmol}), \mathrm{Ag}_{2} \mathrm{O}$ (92.4 mg, $\left.0.4 \mathrm{mmol}\right)$ and $\mathrm{CH}_{3} \mathrm{CN}$ (4 mL). Time of reaction: 12 hours. The crude mixture was purified by silica gel chromatography (ethyl acetate/petroleum ether 1:10) to afford the product 3 as a colorless oil (45.1 $\mathrm{mg}$, $82 \%$ yield). ${ }^{1} \mathrm{H}$ NMR $\left(600 \mathrm{MHz}, \mathrm{CDCl}_{3}\right) \delta 8.39(\mathrm{~d}, J=3.3 \mathrm{~Hz}, 1 \mathrm{H}), 7.77(\mathrm{~d}, J=7.6 \mathrm{~Hz}$, 1H), $7.58(\mathrm{~m}, 4 \mathrm{H}), 7.49(\mathrm{~m}, 3 \mathrm{H}), 7.07(\mathrm{~d}, J=7.6 \mathrm{~Hz}, 2 \mathrm{H}), 7.05-7.00(\mathrm{~m}, 1 \mathrm{H}), 2.31(\mathrm{~s}$, $3 \mathrm{H}) .{ }^{13} \mathrm{C}$ NMR $\left(150 \mathrm{MHz}, \mathrm{CDCl}_{3}\right) \delta 157.3,149.4,143.4,140.1,139.9,136.6,135.6$, $130.3,130.0,129.2,129.2,129.1,128.7,123.1,122.2,21.9$

\section{Scale up reaction for compound 3}

To a $100 \mathrm{~mL}$ flask, containing the 4-methylbenzaldehyde 1 (600.0 mg, $5 \mathrm{mmol})$, 2phenylpyridine 2 (311.1 mg, 2mmol), PQ (42mg, 0.2mmol), Pd(OAc)2 (89.6mg, 0.4mmol), $\mathrm{Ag}_{2} \mathrm{O}$ (924mg, $\left.4 \mathrm{mmol}\right)$ was added $\mathrm{CH}_{3} \mathrm{CN}(40 \mathrm{~mL})$. The flask was then capped under Ar (Insert an Ar ball), vortexed for approximately one minute, and then placed $\sim 7 \mathrm{~cm}$ from two $18 \mathrm{~W}$ blue LED lamps. The reaction temperature was approximately $25^{\circ} \mathrm{C}$, with a fan used to assist cooling. After 24 hours, the reaction was quenched by water $(30 \mathrm{~mL})$, extracted with ethyl acetate $(50 \mathrm{~mL} \times 3)$. The combined organic layers were washed with brine, dried over anhydrous $\mathrm{Na}_{2} \mathrm{SO}_{4}$ and concentrated. The crude product was subjected to column chromatography (ethyl acetate /petroleum ether $=1 / 10$ ) on silica gel to afford $398.6 \mathrm{mg}$ of product 3 in $73 \%$ yield. 


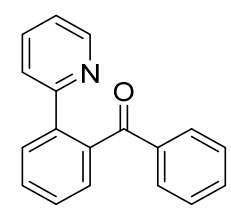

4

\section{Phenyl(2-(pyridin-2-yl)phenyl)methanone (4) ${ }^{1}$}

Prepared according to the general procedure using benzaldehyde $(53.6 \mathrm{mg}, 0.5 \mathrm{mmol})$, 2-phenylpyridine 2 (31.1 mg, $0.2 \mathrm{mmol}), \mathrm{PQ}$ (4.2 mg, $0.02 \mathrm{mmol}), \mathrm{Pd}(\mathrm{OAc}) 2$ (8.9 mg, $0.04 \mathrm{mmol}), \mathrm{Ag}_{2} \mathrm{O}$ (92.4 mg, $\left.0.4 \mathrm{mmol}\right)$ and $\mathrm{CH}_{3} \mathrm{CN}$ (4 mL). Time of reaction: 12 hours. The crude mixture was purified by silica gel chromatography (ethyl acetate/petroleum ether 1:10) to afford the product 4 as a colorless oil (36.3mg, 70\% yield). ${ }^{1} \mathrm{H}$ NMR (600 $\left.\mathrm{MHz}, \mathrm{CDCl}_{3}\right) \delta 8.36(\mathrm{~d}, J=4.6 \mathrm{~Hz}, 1 \mathrm{H}), 7.77(\mathrm{~d}, J=7.7 \mathrm{~Hz}, 1 \mathrm{H}), 7.68(\mathrm{~d}, J=7.7 \mathrm{~Hz}$, 2H), $7.63-7.58(\mathrm{~m}, 1 \mathrm{H}), 7.55(\mathrm{~m}, 3 \mathrm{H}), 7.49(\mathrm{~m}, 1 \mathrm{H}), 7.38(\mathrm{~m}, 1 \mathrm{H}), 7.26(\mathrm{~m}, 2 \mathrm{H}), 7.00$ (m, 1H). ${ }^{13} \mathrm{C} \mathrm{NMR}\left(150 \mathrm{MHz}, \mathrm{CDCl}_{3}\right) \delta 198.4,156.9,149.1,139.8,139.7,138.1,136.4$, $132.4,130.3,129.6,129.2,128.9,128.6,128.1,122.8,122.0$.

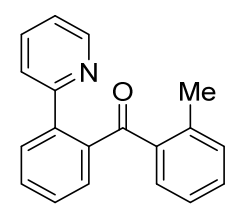

5

\section{(2-Methylphenyl)[2-(2-pyridinyl)phenyl]methanone (5) ${ }^{1}$}

Prepared according to the general procedure using 2-methylbenzaldehyde $(60.0 \mathrm{mg}, 0.5$ mmol), 2-phenylpyridine 2 (31.1 mg, $0.2 \mathrm{mmol})$, PQ (4.2 mg, $0.02 \mathrm{mmol}), \operatorname{Pd}(\mathrm{OAc}) 2$ (8.9 mg, $0.04 \mathrm{mmol}), \mathrm{Ag}_{2} \mathrm{O}$ (92.4 mg, $0.4 \mathrm{mmol}$ ) and $\mathrm{CH}_{3} \mathrm{CN}$ (4 mL). Time of reaction: 12 hours. The crude mixture was purified by silica gel chromatography (ethyl acetate/petroleum ether 1:10) to afford the product as a colorless oil $(31.7 \mathrm{mg}, 58 \%$ yield). ${ }^{1} \mathrm{H} \mathrm{NMR}\left(600 \mathrm{MHz}, \mathrm{CDCl}_{3}\right) \delta 8.42(\mathrm{~d}, J=4.7 \mathrm{~Hz}, 1 \mathrm{H}), 7.63(\mathrm{~m}, 2 \mathrm{H}), 7.59(\mathrm{~m}$, 1H), $7.56-7.49$ (m, 2H), 7.41 (d, J=7.9 Hz, 1H), 7.16 (m, 2H), 7.08 (d, J= $7.5 \mathrm{~Hz}$, 1H), $6.99(\mathrm{~m}, 1 \mathrm{H}), 6.93(\mathrm{~m}, 1 \mathrm{H}), 2.58(\mathrm{~s}, 3 \mathrm{H}) .{ }^{13} \mathrm{C} \mathrm{NMR}\left(150 \mathrm{MHz}, \mathrm{CDCl}_{3}\right) \delta 199.9$, 157.6, 148.9, 140.9, 140.4, 139.3, 138.2, 136.3, 131.3, 131.0, 130.6, 130.6, 129.9, 129.2, 128.6, 124.9, 122.6, 121.9, 21.1. 


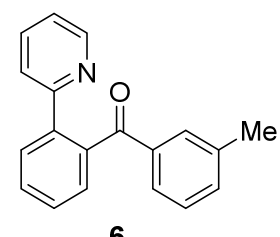

\section{(3-Methylphenyl)[2-(2-pyridinyl)phenyl]methanone (6) ${ }^{2}$}

Prepared according to the general procedure using 3-methylbenzaldehyde (60.0 mg, 0.5 mmol), 2-phenylpyridine 2 (31.1 mg, $0.2 \mathrm{mmol})$, PQ (4.2 mg, $0.02 \mathrm{mmol}), \mathrm{Pd}(\mathrm{OAc})_{2}$ ( $8.9 \mathrm{mg}, 0.04 \mathrm{mmol}), \mathrm{Ag}_{2} \mathrm{O}$ (92.4 mg, $0.4 \mathrm{mmol}$ ) and $\mathrm{CH}_{3} \mathrm{CN}$ (4 mL). Time of reaction: 12 hours. The crude mixture was purified by silica gel chromatography (ethyl acetate/petroleum ether 1:10) to afford the product as a colorless oil $(33.8 \mathrm{mg}, 62 \%$ yield). ${ }^{1} \mathrm{H}$ NMR $\left(600 \mathrm{MHz}, \mathrm{CDCl}_{3}\right) \delta 8.38(\mathrm{~d}, J=4.3 \mathrm{~Hz}, 1 \mathrm{H}), 7.77(\mathrm{~d}, J=7.8 \mathrm{~Hz}, 1 \mathrm{H})$, $7.63-7.58(\mathrm{~m}, 1 \mathrm{H}), 7.58-7.50(\mathrm{~m}, 4 \mathrm{H}), 7.50-7.45(\mathrm{~m}, 2 \mathrm{H}), 7.21(\mathrm{~d}, J=7.5 \mathrm{~Hz}, 1 \mathrm{H})$, $7.15(\mathrm{~m}, 1 \mathrm{H}), 7.02(\mathrm{~m}, 1 \mathrm{H}), 2.28(\mathrm{~s}, 3 \mathrm{H}) .{ }^{13} \mathrm{C} \mathrm{NMR}\left(150 \mathrm{MHz}, \mathrm{CDCl}_{3}\right) \delta$ 198.5, 157.1, $149.2,139.9,139.8,138.0,137.9,136.3,133.3,130.3,130.1,129.2,129.0,128.5,128.1$, $127.1,122.9,122.0,21.3$.

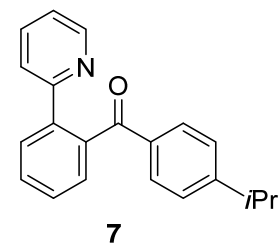

\section{(4-Isopropylphenyl)(2-(pyridin-2-yl)phenyl)methanone (7)}

Prepared according to the general procedure using $p$-isopropylbenzaldehyde $(74.1 \mathrm{mg}$, $0.5 \mathrm{mmol})$, 2-phenylpyridine 2 (31.1 $\mathrm{mg}, 0.2 \mathrm{mmol})$, PQ (4.2 mg, $0.02 \mathrm{mmol})$, $\mathrm{Pd}(\mathrm{OAc})_{2}(8.9 \mathrm{mg}, 0.04 \mathrm{mmol}), \mathrm{Ag}_{2} \mathrm{O}$ (92.4 mg, $\left.0.4 \mathrm{mmol}\right)$ and $\mathrm{CH}_{3} \mathrm{CN}$ (4 mL). Time of reaction: 12 hours. The crude mixture was purified by silica gel chromatography (ethyl acetate/petroleum ether 1:10) to afford the product 7 as a colorless oil (42 $\mathrm{mg}$, 70\% yield). ${ }^{1} \mathrm{H} \mathrm{NMR}\left(400 \mathrm{MHz}, \mathrm{CDCl}_{3}\right) \delta 8.39(\mathrm{~d}, J=4.5 \mathrm{~Hz}, 1 \mathrm{H}), 7.76(\mathrm{~d}, J=7.7 \mathrm{~Hz}$, 1H), $7.63(\mathrm{~m}, 2 \mathrm{H}), 7.61-7.44(\mathrm{~m}, 5 \mathrm{H}), 7.13(\mathrm{~d}, J=8.2 \mathrm{~Hz}, 2 \mathrm{H}), 7.01(\mathrm{~m}, 1 \mathrm{H}), 2.93-$ $2.79(\mathrm{~m}, 1 \mathrm{H}), 1.19(\mathrm{~d}, J=6.9 \mathrm{~Hz}, 6 \mathrm{H}) .{ }^{13} \mathrm{C} \mathrm{NMR}\left(100 \mathrm{MHz}, \mathrm{CDCl}_{3}\right) \delta$ 198.0, 157.2, $153.9,149.2,139.9,139.8,136.3,135.8,130.2,130.0,129.1,128.4,126.3,123.0,121.9$, 
34.3, 23.7. HRMS (ESI) m/z: [M + H] $]^{+}$calcd for $\mathrm{C}_{21} \mathrm{H}_{20} \mathrm{NO} 302.1539$; found 302.1540 .

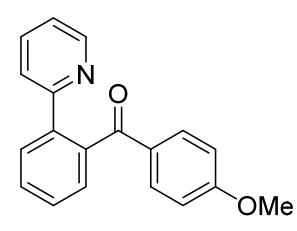

8

\section{(4-Methoxyphenyl)(2-(pyridin-2-yl)phenyl)methanone $(8)^{2}$}

Prepared according to the general procedure using $p$-anisaldehyde $(68.6 \mathrm{mg}, 0.5 \mathrm{mmol})$, 2-phenylpyridine 2 (31.1 mg, $0.2 \mathrm{mmol}), \mathrm{PQ}$ (4.2 mg, $0.02 \mathrm{mmol}), \mathrm{Pd}(\mathrm{OAc}) 2$ (8.9 mg, $0.04 \mathrm{mmol}), \mathrm{Ag}_{2} \mathrm{O}$ (92.4 mg, $\left.0.4 \mathrm{mmol}\right)$ and $\mathrm{CH}_{3} \mathrm{CN}$ (4 mL). Time of reaction: 12 hours. The crude mixture was purified by silica gel chromatography (ethyl acetate/petroleum ether $1: 3)$ to afford the product 8 as a colorless oil (44.5mg, 77\% yield). ${ }^{1} \mathrm{H}$ NMR (600 $\left.\mathrm{MHz}, \mathrm{CDCl}_{3}\right) \delta 8.41(\mathrm{~d}, J=4.2 \mathrm{~Hz}, 1 \mathrm{H}), 7.76(\mathrm{~d}, J=7.7 \mathrm{~Hz}, 1 \mathrm{H}), 7.67(\mathrm{~d}, J=8.8 \mathrm{~Hz}$, 2H), $7.57(\mathrm{~m}, 2 \mathrm{H}), 7.50(\mathrm{~d}, J=4.2 \mathrm{~Hz}, 2 \mathrm{H}), 7.46(\mathrm{~d}, J=7.7 \mathrm{~Hz}, 1 \mathrm{H}), 7.06-7.01(\mathrm{~m}$, $1 \mathrm{H}), 6.76(\mathrm{~d}, J=8.8 \mathrm{~Hz}, 2 \mathrm{H}), 3.78(\mathrm{~s}, 3 \mathrm{H}) .{ }^{13} \mathrm{C} \mathrm{NMR}\left(150 \mathrm{MHz}, \mathrm{CDCl}_{3}\right) \delta 163.2,157.3$, $149.3,139.9,139.6,136.4,132.1,130.9,130.1,129.2,128.9,128.5,123.2,122.1,113.5$, 55.5

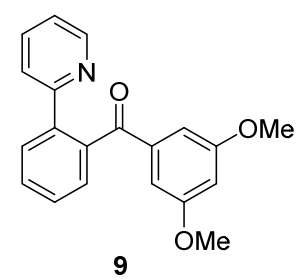

\section{(3,5-Dimethoxyphenyl)(2-(pyridin-2-yl)phenyl)methanone (9)}

Prepared according to the general procedure using 3,5-dimethoxybenzaldehyde $(83.7$ mg, $0.5 \mathrm{mmol})$, 2-phenylpyridine 2 (31.1 mg, $0.2 \mathrm{mmol})$, PQ (4.2 mg, $0.02 \mathrm{mmol})$, $\mathrm{Pd}(\mathrm{OAc})_{2}(8.9 \mathrm{mg}, 0.04 \mathrm{mmol}), \mathrm{Ag}_{2} \mathrm{O}$ (92.4 mg, $\left.0.4 \mathrm{mmol}\right)$ and $\mathrm{CH}_{3} \mathrm{CN}$ (4 mL). Time of reaction: 12 hours. The crude mixture was purified by silica gel chromatography (ethyl acetate/petroleum ether 1:3) to afford the product 9 as a colorless oil (54.3mg, 85\% yield). ${ }^{1} \mathrm{H}$ NMR $\left(600 \mathrm{MHz}, \mathrm{CDCl}_{3}\right) \delta 8.39(\mathrm{~d}, J=4.7 \mathrm{~Hz}, 1 \mathrm{H}), 7.76(\mathrm{~d}, J=7.7 \mathrm{~Hz}$, 1H), $7.62-7.55(\mathrm{~m}, 2 \mathrm{H}), 7.54-7.47(\mathrm{~m}, 3 \mathrm{H}), 7.04(\mathrm{~m}, 1 \mathrm{H}), 6.85(\mathrm{~d}, J=2.2 \mathrm{~Hz}, 2 \mathrm{H})$, 
$6.49(\mathrm{~m}, 1 \mathrm{H}), 3.72(\mathrm{~s}, 6 \mathrm{H}) .{ }^{13} \mathrm{C} \mathrm{NMR}\left(150 \mathrm{MHz}, \mathrm{CDCl}_{3}\right) \delta 160.5,156.9,149.1,140.0$, $139.7,139.5,136.4,130.3,129.24128 .8,128.6,122.6,122.1,107.5,105.2$, 55.6. HRMS (ESI) m/z: [M + H $]^{+}$calcd. for $\mathrm{C}_{20} \mathrm{H}_{18} \mathrm{NO}_{3}$ 320.1281; found 320.1279.

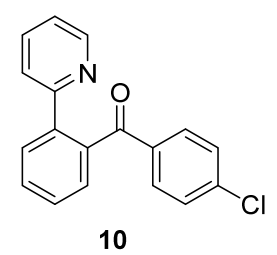

\section{(4-Chlorophenyl)(2-(pyridin-2-yl)phenyl)methanone $(10)^{1}$}

Prepared according to the general procedure using 4-chlorobenzaldehyde (70.0 mg, 0.5 mmol), 2-phenylpyridine 2 (31.1 mg, $0.2 \mathrm{mmol})$, PQ (4.2 mg, $0.02 \mathrm{mmol}), \mathrm{Pd}(\mathrm{OAc})_{2}$ ( $8.9 \mathrm{mg}, 0.04 \mathrm{mmol}), \mathrm{Ag}_{2} \mathrm{O}$ (92.4 mg, $\left.0.4 \mathrm{mmol}\right)$ and $\mathrm{CH}_{3} \mathrm{CN}$ (4 mL). Time of reaction: 12 hours. The crude mixture was purified by silica gel chromatography (ethyl acetate/petroleum ether $1: 10)$ to afford the product $\mathbf{1 0}$ as a colorless oil $(44.5 \mathrm{mg}, 76 \%$ yield). ${ }^{1} \mathrm{H} \mathrm{NMR}\left(400 \mathrm{MHz}, \mathrm{CDCl}_{3}\right) \delta 8.33(\mathrm{~d}, J=4.4 \mathrm{~Hz}, 1 \mathrm{H}), 7.77(\mathrm{~d}, J=7.7 \mathrm{~Hz}, 1 \mathrm{H})$, $7.60(\mathrm{~m}, 4 \mathrm{H}), 7.52(\mathrm{~m}, 3 \mathrm{H}), 7.25-7.19(\mathrm{~m}, 2 \mathrm{H}), 7.03(\mathrm{~m}, 1 \mathrm{H}) .{ }^{13} \mathrm{C}$ NMR (100 MHz, $\left.\mathrm{CDCl}_{3}\right) \delta 197.1,156.5,149.1,139.6,139.2,138.6,136.6,136.6,130.8,130.5,129.1$, $128.8,128.7,128.5,122.5,122.2$.

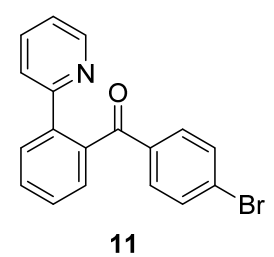

\section{(4-Bromophenyl)(2-(pyridin-2-yl)phenyl)methanone (11) ${ }^{1}$}

Prepared according to the general procedure using 4-bromobenzaldehyde ( $92.5 \mathrm{mg}, 0.5$ mmol), 2-phenylpyridine 2 (31.1 mg, $0.2 \mathrm{mmol})$, PQ (4.2 mg, $0.02 \mathrm{mmol}), \mathrm{Pd}(\mathrm{OAc}) 2$ (8.9 mg, $0.04 \mathrm{mmol}), \mathrm{Ag}_{2} \mathrm{O}$ (92.4 mg, $0.4 \mathrm{mmol}$ ) and $\mathrm{CH}_{3} \mathrm{CN}$ (4 mL). Time of reaction: 12 hours. The crude mixture was purified by silica gel chromatography (ethyl acetate/petroleum ether $1: 10)$ to afford the product $\mathbf{1 1}$ as a colorless oil $(52.6 \mathrm{mg}, 78 \%$ 
yield). ${ }^{1} \mathrm{H}$ NMR (600 MHz, $\left.\mathrm{CDCl}_{3}\right) \delta 8.33(\mathrm{~d}, J=4.4 \mathrm{~Hz}, 1 \mathrm{H}), 7.77(\mathrm{~d}, J=7.7 \mathrm{~Hz}, 1 \mathrm{H})$, $7.64-7.57$ (m, 2H), $7.57-7.49(\mathrm{~m}, 5 \mathrm{H}), 7.43-7.36(\mathrm{~m}, 2 \mathrm{H}), 7.08-7.00(\mathrm{~m}, 1 \mathrm{H}) .{ }^{13} \mathrm{C}$ $\operatorname{NMR}\left(150 \mathrm{MHz}, \mathrm{CDCl}_{3}\right) \delta 156.5,149.1,139.5,139.2,137.0,136.6,131.4,130.9,130.5$, $129.1,128.8,128.7,127.4,122.5,122.2$.

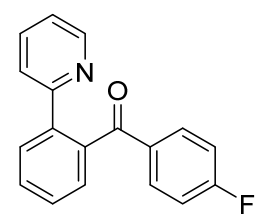

12

\section{(4-Fluorophenyl)(2-(pyridin-2-yl)phenyl)methanone (12) ${ }^{2}$}

Prepared according to the general procedure using 4-fluorobenzaldehyde $(62.4 \mathrm{mg}, 0.5$ mmol), 2-phenylpyridine 2 (31.1 mg, $0.2 \mathrm{mmol})$, PQ (4.2 mg, $0.02 \mathrm{mmol}$ ), $\mathrm{Pd}(\mathrm{OAc})_{2}$ ( $8.9 \mathrm{mg}, 0.04 \mathrm{mmol}), \mathrm{Ag}_{2} \mathrm{O}(92.4 \mathrm{mg}, 0.4 \mathrm{mmol})$ and $\mathrm{CH}_{3} \mathrm{CN}(4 \mathrm{~mL})$. Time of reaction: 12 hours. The crude mixture was purified by silica gel chromatography (ethyl acetate/petroleum ether 1:10) to afford the product $\mathbf{1 2}$ as a colorless oil $(39.3 \mathrm{mg}, 71 \%$ yield). ${ }^{1} \mathrm{H} \mathrm{NMR}\left(600 \mathrm{MHz}, \mathrm{CDCl}_{3}\right) \delta 8.37(\mathrm{~d}, J=4.4 \mathrm{~Hz}, 1 \mathrm{H}), 7.77(\mathrm{~d}, J=7.7 \mathrm{~Hz}, 1 \mathrm{H})$, $7.70(\mathrm{dd}, J=8.1,5.7 \mathrm{~Hz}, 2 \mathrm{H}), 7.61(\mathrm{~m}, 2 \mathrm{H}), 7.52(\mathrm{~m}, 3 \mathrm{H}), 7.08-7.02(\mathrm{~m}, 1 \mathrm{H}), 6.93$ (m, 2H). ${ }^{13} \mathrm{C}$ NMR $\left(150 \mathrm{MHz}, \mathrm{CDCl}_{3}\right) \delta 165.31\left(\mathrm{~d},{ }^{1} J_{\mathrm{C}-\mathrm{F}}=252.0 \mathrm{~Hz}\right), 156.8,149.1$, $139.6,139.4,136.6,134.6\left(\mathrm{~d},{ }^{4} J_{\mathrm{C}-\mathrm{F}}=3.0 \mathrm{~Hz}\right), 132.1\left(\mathrm{~d},{ }^{3} J_{\mathrm{C}-\mathrm{F}}=9.0 \mathrm{~Hz}\right), 130.4,129.1$, $128.9,128.7,122.8,122.2,115.3\left(\mathrm{~d},{ }^{2} J_{\mathrm{C}-\mathrm{F}}=22.5 \mathrm{~Hz}\right) .{ }^{19} \mathrm{~F} \mathrm{NMR}\left(565 \mathrm{MHz}, \mathrm{CDCl}_{3}\right) \delta-$ 106.29 .

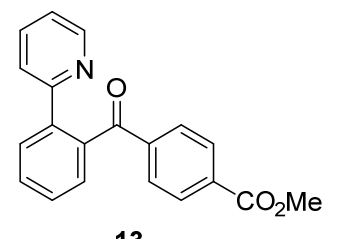

13

\section{Methyl 4-(2-(pyridin-2-yl)benzoyl)benzoate (13)}

Prepared according to the general procedure using 4-(methoxycarbonyl)benzaldehyde (82.0 mg, 0.5 mmol), 2-phenylpyridine 2 (31.1 mg, $0.2 \mathrm{mmol})$, PQ (4.2 mg, $0.02 \mathrm{mmol}$ ), $\mathrm{Pd}(\mathrm{OAc})_{2}(8.9 \mathrm{mg}, 0.04 \mathrm{mmol}), \mathrm{Ag}_{2} \mathrm{O}$ (92.4 mg, $\left.0.4 \mathrm{mmol}\right)$ and $\mathrm{CH}_{3} \mathrm{CN}(4 \mathrm{~mL})$. Time 
of reaction: 12 hours. The crude mixture was purified by silica gel chromatography (ethyl acetate/petroleum ether 1:6) to afford the product $\mathbf{1 3}$ as a colorless oil $(51.4 \mathrm{mg}$, 81\% yield). ${ }^{1} \mathrm{H}$ NMR $\left(600 \mathrm{MHz}, \mathrm{CDCl}_{3}\right) \delta 8.29(\mathrm{~d}, J=4.6 \mathrm{~Hz}, 1 \mathrm{H}), 7.90(\mathrm{~d}, J=8.4 \mathrm{~Hz}$, 2H), $7.78(\mathrm{~d}, J=7.8 \mathrm{~Hz}, 1 \mathrm{H}), 7.70(\mathrm{~d}, J=8.4 \mathrm{~Hz}, 2 \mathrm{H}), 7.63(\mathrm{~m}, 1 \mathrm{H}), 7.60-7.52(\mathrm{~m}$, 4H), $7.03-6.95(\mathrm{~m}, 1 \mathrm{H}), 3.89$ (s, 3H). ${ }^{13} \mathrm{C} \mathrm{NMR}\left(150 \mathrm{MHz}, \mathrm{CDCl}_{3}\right) \delta$ 197.5, 166.5, 156.4, 149.0, 141.8, 139.6, 139.3, 136.7, 133.0, 130.6, 129.4, 129.4, 129.1, 128.9, 128.6, 122.4, 122.3, 52.4. HRMS (ESI) m/z: [M+ H] $]^{+}$calcd. for $\mathrm{C}_{20} \mathrm{H}_{16} \mathrm{NO}_{3}$ 318.1125; found 318.1128 .

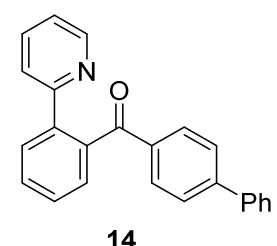

\section{[1,1'-Biphenyl]-4-yl(2-(pyridin-2-yl)phenyl)methanone (14) ${ }^{2}$}

Prepared according to the general procedure using 4-biphenylcarboxaldehyde $(91.1 \mathrm{mg}$, $0.5 \mathrm{mmol}$ ), 2-phenylpyridine 2 (31.1 $\mathrm{mg}, 0.2 \mathrm{mmol})$, PQ (4.2 mg, $0.02 \mathrm{mmol}$ ), $\mathrm{Pd}(\mathrm{OAc})_{2}(8.9 \mathrm{mg}, 0.04 \mathrm{mmol}), \mathrm{Ag}_{2} \mathrm{O}(92.4 \mathrm{mg}, 0.4 \mathrm{mmol})$ and $\mathrm{CH}_{3} \mathrm{CN}$ (4 mL). Time of reaction: 12 hours. The crude mixture was purified by silica gel chromatography (ethyl acetate/petroleum ether 1:6) to afford the product $\mathbf{1 4}$ as a colorless oil (58.9 $\mathrm{mg}$, $88 \%$ yield). ${ }^{1} \mathrm{H}$ NMR $\left(600 \mathrm{MHz}, \mathrm{CDCl}_{3}\right) \delta 8.39(\mathrm{~d}, J=4.4 \mathrm{~Hz}, 1 \mathrm{H}), 7.79(\mathrm{~m}, 3 \mathrm{H}), 7.62$ (m, 1H), $7.59-7.49(\mathrm{~m}, 8 \mathrm{H}), 7.43(\mathrm{~m}, 2 \mathrm{H}), 7.36(\mathrm{~m}, 1 \mathrm{H}), 7.04-6.99(\mathrm{~m}, 1 \mathrm{H}) .{ }^{13} \mathrm{C}$ $\operatorname{NMR}\left(150 \mathrm{MHz}, \mathrm{CDCl}_{3}\right) \delta 197.8,156.9,149.2,145.0,140.1,139.7,139.7,136.8,136.4$, $130.3,130.2,129.1,128.9,128.9,128.6,128.2,127.3,126.8,122.8,122.1$.

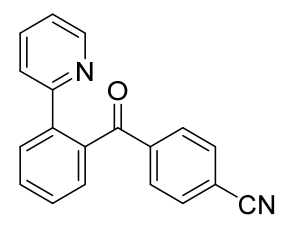

15

\section{4-(2-(Pyridin-2-yl)benzoyl)benzonitrile (15) ${ }^{1}$}

Prepared according to the general procedure using 4-cyanobenzaldehyde (65.5 mg, 0.5 
mmol), 2-phenylpyridine 2 (31.1 mg, $0.2 \mathrm{mmol})$, PQ (4.2 mg, $0.02 \mathrm{mmol}), \mathrm{Pd}(\mathrm{OAc})_{2}$ (8.9 mg, $0.04 \mathrm{mmol}), \mathrm{Ag}_{2} \mathrm{O}$ (92.4 mg, $\left.0.4 \mathrm{mmol}\right)$ and $\mathrm{CH}_{3} \mathrm{CN}$ (4 mL). Time of reaction: 12 hours. The crude mixture was purified by silica gel chromatography (ethyl acetate/petroleum ether 1:3) to afford the product 15 as a colorless oil $(36.3 \mathrm{mg}, 64 \%$ yield). ${ }^{1} \mathrm{H} \mathrm{NMR}\left(600 \mathrm{MHz}, \mathrm{CDCl}_{3}\right) \delta 8.24(\mathrm{~d}, J=4.4 \mathrm{~Hz}, 1 \mathrm{H}), 7.79(\mathrm{~d}, J=7.7 \mathrm{~Hz}, 1 \mathrm{H})$, $7.72(\mathrm{~d}, J=8.3 \mathrm{~Hz}, 2 \mathrm{H}), 7.65-7.56(\mathrm{~m}, 3 \mathrm{H}), 7.56-7.50(\mathrm{~m}, 4 \mathrm{H}), 7.06-6.94(\mathrm{~m}, 1 \mathrm{H})$. ${ }^{13} \mathrm{C} \mathrm{NMR}\left(150 \mathrm{MHz}, \mathrm{CDCl}_{3}\right) \delta 196.5,155.9,148.8,141.8,139.3,138.6,136.8,132.0$, $130.8,129.3,129.3,129.1,128.4,122.4,122.1,118.2,115.2$.

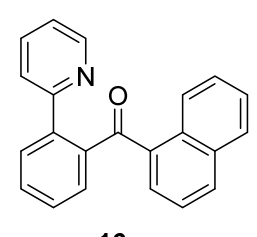

16

\section{Naphthalen-1-yl(2-(pyridin-2-yl)phenyl)methanone (16) ${ }^{1}$}

Prepared according to the general procedure using 1-naphthaldehyde (78.1 mg, 0.5 mmol), 2-phenylpyridine 2 (31.1 mg, $0.2 \mathrm{mmol})$, PQ (4.2 mg, $0.02 \mathrm{mmol}), \mathrm{Pd}(\mathrm{OAc}) 2$ (8.9 mg, $0.04 \mathrm{mmol}), \mathrm{Ag}_{2} \mathrm{O}$ (92.4 mg, $\left.0.4 \mathrm{mmol}\right)$ and $\mathrm{CH}_{3} \mathrm{CN}(4 \mathrm{~mL})$. Time of reaction: 12 hours. The crude mixture was purified by silica gel chromatography (ethyl acetate/petroleum ether 1:10) to afford the product 16as a colorless oil $(53.8 \mathrm{mg}, 87 \%$ yield). ${ }^{1} \mathrm{H} \mathrm{NMR}\left(600 \mathrm{MHz}, \mathrm{CDCl}_{3}\right) \delta 8.91(\mathrm{~d}, J=8.6 \mathrm{~Hz}, 1 \mathrm{H}), 8.12(\mathrm{~d}, J=4.5 \mathrm{~Hz}, 1 \mathrm{H})$, $7.74(\mathrm{~m}, 4 \mathrm{H}), 7.63(\mathrm{~m}, 2 \mathrm{H}), 7.55(\mathrm{~m}, 1 \mathrm{H}), 7.51(\mathrm{~m}, 1 \mathrm{H}), 7.45-7.31(\mathrm{~m}, 3 \mathrm{H}), 7.16(\mathrm{~m}$, 1H), $6.81-6.70(\mathrm{~m}, 1 \mathrm{H}) .{ }^{13} \mathrm{C} \mathrm{NMR}\left(150 \mathrm{MHz}, \mathrm{CDCl}_{3}\right) \delta 199.7,157.2,149.1,141.3$, $140.5,136.5,136.2,133.7,132.4,131.2,130.7,130.3,129.8,129.0,128.6,128.1,127.7$, $126.7,126.3,123.9,122.3,121.5$.

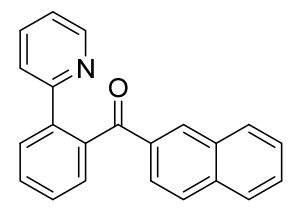

17

\section{Naphthalen-2-yl(2-(pyridin-2-yl)phenyl)methanone $(17)^{2}$}


Prepared according to the general procedure using 2-naphthaldehyde $(78.1 \mathrm{mg}, 0.5$ mmol), 2-phenylpyridine 2 (31.1 mg, $0.2 \mathrm{mmol})$, PQ (4.2 mg, $0.02 \mathrm{mmol}), \operatorname{Pd}(\mathrm{OAc}) 2$ (8.9 mg, $0.04 \mathrm{mmol}), \mathrm{Ag}_{2} \mathrm{O}$ (92.4 mg, $0.4 \mathrm{mmol}$ ) and $\mathrm{CH}_{3} \mathrm{CN}$ (4 mL). Time of reaction: 12 hours. The crude mixture was purified by silica gel chromatography (ethyl acetate/petroleum ether 1:9) to afford the product $\mathbf{1 7}$ as a colorless oil $(50.7 \mathrm{mg}, 82 \%$ yield). ${ }^{1} \mathrm{H}$ NMR (400 MHz, $\left.\mathrm{CDCl}_{3}\right) \delta 8.31$ (d, J=4.7 Hz, 1H), 8.08 (s, 1H), 7.93 (dd, $J=8.6,1.5 \mathrm{~Hz}, 1 \mathrm{H}), 7.84-7.74(\mathrm{~m}, 4 \mathrm{H}), 7.68-7.62(\mathrm{~m}, 1 \mathrm{H}), 7.62-7.55(\mathrm{~m}, 2 \mathrm{H})$, $7.55-7.49(\mathrm{~m}, 3 \mathrm{H}), 7.44(\mathrm{~m}, 1 \mathrm{H}), 6.92(\mathrm{~m}, 1 \mathrm{H}) .{ }^{13} \mathrm{C} \mathrm{NMR}\left(100 \mathrm{MHz}, \mathrm{CDCl}_{3}\right) \delta 198.3$, 156.9, 149.1, 139.8, 139.8, 136.4, 135.5, 135.3, 132.4, 131.6, 130.3, 129.6, 129.3, 129.0, $128.6,128.3,128.1,127.8,126.5,125.1,122.6,122.0$.

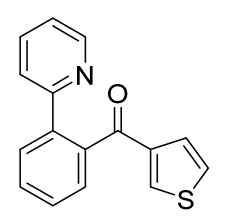

18

\section{(2-(Pyridin-2-yl)phenyl)(thiophen-3-yl)methanone (18)}

Prepared according to the general procedure using 3-thiophenecarboxaldehyde (56.1 $\mathrm{mg}, 0.5 \mathrm{mmol})$, 2-phenylpyridine 2 (31.1 mg, $0.2 \mathrm{mmol})$, PQ (4.2 mg, $0.02 \mathrm{mmol})$, $\mathrm{Pd}(\mathrm{OAc})_{2}(8.9 \mathrm{mg}, 0.04 \mathrm{mmol}), \mathrm{Ag}_{2} \mathrm{O}(92.4 \mathrm{mg}, 0.4 \mathrm{mmol})$ and $\mathrm{CH}_{3} \mathrm{CN}(4 \mathrm{~mL})$. Time of reaction: 12 hours. The crude mixture was purified by silica gel chromatography (ethyl acetate/petroleum ether 1:4) to afford the product $\mathbf{1 8}$ as a colorless oil (42.9 $\mathrm{mg}$, 81\% yield). ${ }^{1} \mathrm{H}$ NMR (600 MHz, $\left.\mathrm{CDCl}_{3}\right) \delta 8.45$ (d, $\left.J=4.4 \mathrm{~Hz}, 1 \mathrm{H}\right), 7.76$ (d, $J=7.7 \mathrm{~Hz}$, 1H), $7.67-7.54(\mathrm{~m}, 4 \mathrm{H}), 7.53-7.45(\mathrm{~m}, 2 \mathrm{H}), 7.41(\mathrm{~d}, J=4.9 \mathrm{~Hz}, 1 \mathrm{H}), 7.15(\mathrm{dd}, J=$ 4.9, $2.9 \mathrm{~Hz}, 1 \mathrm{H}), 7.10-7.03(\mathrm{~m}, 1 \mathrm{H}) .{ }^{13} \mathrm{C} \mathrm{NMR}\left(150 \mathrm{MHz}, \mathrm{CDCl}_{3}\right) \delta 191.9,157.3$, 149.4, 143.0, 140.3, 139.6, 136.4, 134.0, 130.4, 129.3, 128.9, 128.6, 127.8, 125.9, 123.1, 122.1. HRMS (ESI) m/z: $[\mathrm{M}+\mathrm{H}]^{+}$calcd. for $\mathrm{C}_{16} \mathrm{H}_{12} \mathrm{NOS} 266.0634$; found 266.0631 . 


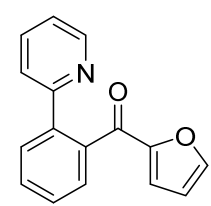

19

\section{Furan-2-yl(2-(pyridin-2-yl)phenyl)methanone (19) ${ }^{3}$}

Prepared according to the general procedure using furfural (48 $\mathrm{mg}, 0.5 \mathrm{mmol}), 2$ phenylpyridine 2 (31.1 mg, $0.2 \mathrm{mmol}), \mathrm{PQ}$ (4.2 mg, $0.02 \mathrm{mmol}), \mathrm{Pd}(\mathrm{OAc}) 2(8.9 \mathrm{mg}$, $0.04 \mathrm{mmol}), \mathrm{Ag}_{2} \mathrm{O}(92.4 \mathrm{mg}, 0.4 \mathrm{mmol})$ and $\mathrm{CH}_{3} \mathrm{CN}(4 \mathrm{~mL})$. Time of reaction: 12 hours. The crude mixture was purified by silica gel chromatography (ethyl acetate/petroleum ether 1:3) to afford the product 19 as a colorless oil (44.8 $\mathrm{mg}, 90 \%$ yield). ${ }^{1} \mathrm{H}$ NMR $\left(600 \mathrm{MHz}, \mathrm{CDCl}_{3}\right) \delta 8.46(\mathrm{~d}, J=4.4 \mathrm{~Hz}, 1 \mathrm{H}), 7.75(\mathrm{~d}, J=7.7 \mathrm{~Hz}, 1 \mathrm{H}), 7.67-7.55(\mathrm{~m}$, 3H), $7.50(\mathrm{~m}, 2 \mathrm{H}), 7.43-7.37(\mathrm{~m}, 1 \mathrm{H}), 7.08(\mathrm{dd}, J=7.0,5.3 \mathrm{~Hz}, 1 \mathrm{H}), 6.79$ (d, $J=3.4$ $\mathrm{Hz}, 1 \mathrm{H}), 6.32(\mathrm{dd}, J=3.5,1.6 \mathrm{~Hz}, 1 \mathrm{H}) .{ }^{13} \mathrm{C} \mathrm{NMR}\left(150 \mathrm{MHz}, \mathrm{CDCl}_{3}\right) \delta 185.4,157.2$, $153.1,149.3,146.5,139.9,138.5,136.5,130.7,129.2,129.1,128.5,122.8,122.0,119.3$, 112.0 .

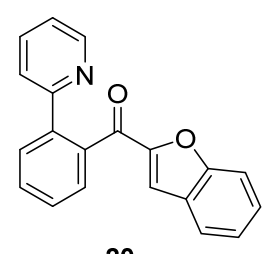

20

\section{Benzofuran-2-yl(2-(pyridin-2-yl)phenyl)methanone (20)}

Prepared according to the general procedure using 2-benzofurancarboxaldehyde (73.0 mg, $0.5 \mathrm{mmol})$, 2-phenylpyridine 2 (31.1 mg, $0.2 \mathrm{mmol})$, PQ (4.2 mg, $0.02 \mathrm{mmol})$, $\mathrm{Pd}(\mathrm{OAc})_{2}(8.9 \mathrm{mg}, 0.04 \mathrm{mmol}), \mathrm{Ag}_{2} \mathrm{O}$ (92.4 mg, $\left.0.4 \mathrm{mmol}\right)$ and $\mathrm{CH}_{3} \mathrm{CN}$ (4 mL). Time of reaction: 12 hours. The crude mixture was purified by silica gel chromatography (ethyl acetate/petroleum ether 1:5) to afford the product $\mathbf{2 0}$ as a colorless oil (46.0 $\mathrm{mg}$, 77\% yield). ${ }^{1} \mathrm{H}$ NMR $\left(600 \mathrm{MHz}, \mathrm{CDCl}_{3}\right) \delta 8.46-8.37(\mathrm{~m}, 1 \mathrm{H}), 7.81(\mathrm{~d}, J=7.8 \mathrm{~Hz}$, 1H), $7.65(\mathrm{~m}, 2 \mathrm{H}), 7.60(\mathrm{~d}, J=3.6 \mathrm{~Hz}, 2 \mathrm{H}), 7.57-7.51(\mathrm{~m}, 2 \mathrm{H}), 7.47(\mathrm{~d}, J=8.4 \mathrm{~Hz}$, 1H), $7.38(\mathrm{dd}, J=8.2,7.3 \mathrm{~Hz}, 1 \mathrm{H}), 7.21(\mathrm{~m}, 1 \mathrm{H}), 7.08(\mathrm{~s}, 1 \mathrm{H}), 7.05-6.97(\mathrm{~m}, 1 \mathrm{H}) .{ }^{13} \mathrm{C}$ NMR $\left(150 \mathrm{MHz}, \mathrm{CDCl}_{3}\right) \delta 187.3,156.9,155.8,153.3,149.2,140.0,138.5,136.6,130.9$, 
129.3, 129.1, 128.7, 128.0, 127.2, 123.7, 123.2, 122.5, 122.2, 115.0, 112.4. HRMS (ESI) $\mathrm{m} / \mathrm{z}:[\mathrm{M}+\mathrm{H}]^{+}$calcd. for $\mathrm{C}_{20} \mathrm{H}_{14} \mathrm{NO}_{2}$ 300.1019; found 300.1017 .

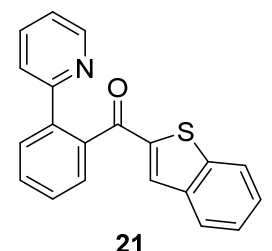

\section{Benzo[b]thiophen-2-yl(2-(pyridin-2-yl)phenyl)methanone (21)}

Prepared according to the general procedure using 2-formylbenzo[b]thiophene $(81.1$ mg, $0.5 \mathrm{mmol})$, 2-phenylpyridine 2 (31.1 mg, $0.2 \mathrm{mmol})$, PQ (4.2 mg, $0.02 \mathrm{mmol})$, $\mathrm{Pd}(\mathrm{OAc})_{2}(8.9 \mathrm{mg}, 0.04 \mathrm{mmol}), \mathrm{Ag}_{2} \mathrm{O}(92.4 \mathrm{mg}, 0.4 \mathrm{mmol})$ and $\mathrm{CH}_{3} \mathrm{CN}$ (4 mL). Time of reaction: 12 hours. The crude mixture was purified by silica gel chromatography (ethyl acetate/petroleum ether 1:4) to afford the product $\mathbf{2 1}$ as a colorless oil (60.0 $\mathrm{mg}$, $96 \%$ yield). ${ }^{1} \mathrm{H}$ NMR $\left(600 \mathrm{MHz}, \mathrm{CDCl}_{3}\right) \delta 8.43(\mathrm{~d}, J=4.7 \mathrm{~Hz}, 1 \mathrm{H}), 7.84-7.79(\mathrm{~m}$, 2H), $7.69-7.62(\mathrm{~m}, 3 \mathrm{H}), 7.61-7.52(\mathrm{~m}, 3 \mathrm{H}), 7.41(\mathrm{~s}, 1 \mathrm{H}), 7.38(\mathrm{~m}, 1 \mathrm{H}), 7.30(\mathrm{~m}, 1 \mathrm{H})$, $7.04-6.99(\mathrm{~m}, 1 \mathrm{H}) .{ }^{13} \mathrm{C} \mathrm{NMR}\left(150 \mathrm{MHz}, \mathrm{CDCl}_{3}\right) \delta 191.9,156.8,149.4,145.0,142.8$, 139.6, 139.1, 136.6, 131.6, 130.6, 129.2, 128.9, 128.6, 127.2, 126.0, 124.8, 122.9, 122.7, 122.2. HRMS (ESI) m/z: $[\mathrm{M}+\mathrm{H}]^{+}$calcd. for $\mathrm{C}_{20} \mathrm{H}_{14} \mathrm{NOS} 316.0791$, found 316.0794 .

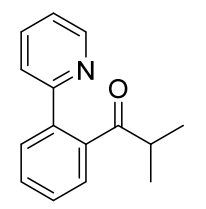

22

\section{2-Methyl-1-(2-(pyridin-2-yl)phenyl)propan-1-one (22)}

Prepared according to the general procedure using isobutyraldehyde (36 mg, $0.5 \mathrm{mmol}$ ), 2-phenylpyridine 2 (31.1 mg, $0.2 \mathrm{mmol}), \mathrm{PQ}$ (4.2 mg, $0.02 \mathrm{mmol}), \mathrm{Pd}(\mathrm{OAc}) 2$ (8.9 mg, $0.04 \mathrm{mmol}), \mathrm{Ag}_{2} \mathrm{O}$ (92.4 mg, $\left.0.4 \mathrm{mmol}\right)$ and $\mathrm{CH}_{3} \mathrm{CN}(4 \mathrm{~mL})$. Time of reaction: 12 hours. The crude mixture was purified by silica gel chromatography (ethyl acetate/petroleum ether 1:3) to afford the product 22 as a colorless oil (24.3 $\mathrm{mg}, 54 \%$ yield). ${ }^{1} \mathrm{H}$ NMR $\left(600 \mathrm{MHz}, \mathrm{CDCl}_{3}\right) \delta 8.65(\mathrm{~d}, J=3.6 \mathrm{~Hz}, 1 \mathrm{H}), 7.77(\mathrm{~m}, 1 \mathrm{H}), 7.69(\mathrm{~d}, J=7.7 \mathrm{~Hz}, 1 \mathrm{H})$, $7.61(\mathrm{~d}, J=7.9 \mathrm{~Hz}, 1 \mathrm{H}), 7.51(\mathrm{~m}, 1 \mathrm{H}), 7.46(\mathrm{~m}, 1 \mathrm{H}), 7.42(\mathrm{~d}, J=7.5 \mathrm{~Hz}, 1 \mathrm{H}), 7.24(\mathrm{dd}$, 
$J=7.1,5.1 \mathrm{~Hz}, 1 \mathrm{H}), 2.54(\mathrm{~m}, 1 \mathrm{H}), 1.03(\mathrm{~d}, J=6.9 \mathrm{~Hz}, 6 \mathrm{H}) .{ }^{13} \mathrm{C} \mathrm{NMR}\left(150 \mathrm{MHz}, \mathrm{CDCl}_{3}\right)$ $\delta 157.3,149.4,141.4,138.2,137.0,130.0,128.8,128.3,122.4,122.3,40.8,18.9$. HRMS (ESI) m/z: [M + H $]^{+}$calcd. for $\mathrm{C}_{15} \mathrm{H}_{16} \mathrm{NO}$ 226.1226, found 226.1226.

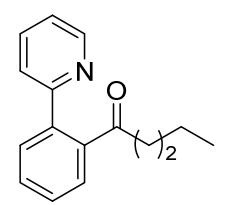

23

\section{1-(2-(Pyridin-2-yl)phenyl)butan-1-one (23) ${ }^{4}$}

Prepared according to the general procedure using valeraldehyde (43.0 $\mathrm{mg}, 0.5 \mathrm{mmol})$, 2-phenylpyridine 2 (31.1 mg, $0.2 \mathrm{mmol}), \mathrm{PQ}$ (4.2 mg, $0.02 \mathrm{mmol}), \mathrm{Pd}(\mathrm{OAc})_{2}(8.9 \mathrm{mg}$, $0.04 \mathrm{mmol}), \mathrm{Ag}_{2} \mathrm{O}$ (92.4 mg, $\left.0.4 \mathrm{mmol}\right)$ and $\mathrm{CH}_{3} \mathrm{CN}$ (4 mL). Time of reaction: 12 hours. The crude mixture was purified by silica gel chromatography (ethyl acetate/petroleum ether 1:2) to afford the product 23 as a colorless oil (34.4 mg, 72\% yield). ${ }^{1} \mathrm{H}$ NMR $\left(600 \mathrm{MHz}, \mathrm{CDCl}_{3}\right) \delta 8.61(\mathrm{~d}, J=4.3 \mathrm{~Hz}, 1 \mathrm{H}), 7.79-7.71(\mathrm{~m}, 1 \mathrm{H}), 7.63(\mathrm{~d}, J=7.6 \mathrm{~Hz}$, 1H), $7.60-7.55(\mathrm{~m}, 1 \mathrm{H}), 7.52-7.47(\mathrm{~m}, 1 \mathrm{H}), 7.47-7.41(\mathrm{~m}, 2 \mathrm{H}), 7.23(\mathrm{~m}, 1 \mathrm{H}), 2.46$ (t, $J=7.5 \mathrm{~Hz}, 2 \mathrm{H}), 1.57(\mathrm{~m}, 2 \mathrm{H}), 1.24(\mathrm{~m}, 2 \mathrm{H}), 0.81(\mathrm{t}, J=7.3 \mathrm{~Hz}, 3 \mathrm{H}) .{ }^{13} \mathrm{C}$ NMR (150 $\left.\mathrm{MHz}, \mathrm{CDCl}_{3}\right) \delta 207.4,157.6,149.3,142.1,138.5,136.8,130.0,129.1,128.7,127.5$, $122.5,122.3,42.9,26.7,22.3,13.9$.

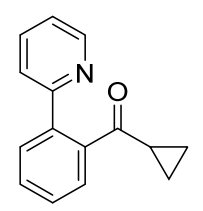

24

\section{Cyclopropyl(2-(pyridin-2-yl)phenyl)methanone (24) ${ }^{2}$}

Prepared according to the general procedure using cyclopropanecarboxaldehyde (35 mg, $0.5 \mathrm{mmol})$, 2-phenylpyridine 2 (31.1 mg, $0.2 \mathrm{mmol})$, PQ (4.2 mg, $0.02 \mathrm{mmol})$, $\mathrm{Pd}(\mathrm{OAc})_{2}(8.9 \mathrm{mg}, 0.04 \mathrm{mmol}), \mathrm{Ag}_{2} \mathrm{O}$ (92.4 mg, $\left.0.4 \mathrm{mmol}\right)$ and $\mathrm{CH}_{3} \mathrm{CN}$ (4 mL). Time of reaction: 12 hours. The crude mixture was purified by silica gel chromatography (ethyl acetate/petroleum ether 1:3) to afford the product $\mathbf{2 4}$ as a colorless oil (30.3 $\mathrm{mg}$, 
68\% yield). ${ }^{1} \mathrm{H}$ NMR (600 MHz, $\left.\mathrm{CDCl}_{3}\right) \delta 8.65(\mathrm{~d}, J=4.6 \mathrm{~Hz}, 1 \mathrm{H}), 7.73(\mathrm{~m}, 1 \mathrm{H}), 7.64$ $(\mathrm{d}, J=7.5 \mathrm{~Hz}, 1 \mathrm{H}), 7.58(\mathrm{~d}, J=7.6 \mathrm{~Hz}, 1 \mathrm{H}), 7.55-7.49$ (m, 2H), $7.49-7.44(\mathrm{~m}, 1 \mathrm{H})$, $7.24(\mathrm{dd}, J=7.1,5.3 \mathrm{~Hz}, 1 \mathrm{H}), 1.82(\mathrm{~m}, 1 \mathrm{H}), 1.12(\mathrm{~m}, 2 \mathrm{H}), 0.73(\mathrm{~m}, 2 \mathrm{H}) .{ }^{13} \mathrm{C}$ NMR $(150$ $\left.\mathrm{MHz}_{2} \mathrm{CDCl}_{3}\right) \delta 206.5,158.1,149.5,141.9,139.4,136.5,130.5,129.5,128.7,128.0$, $123.5,122.3,22.5,12.9$.

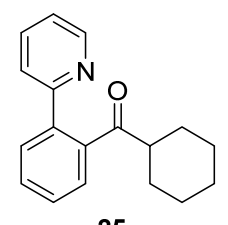

25

\section{Cyclohexyl(2-(pyridin-2-yl)phenyl)methanone (25) ${ }^{4}$}

Prepared according to the general procedure using cyclohexanecarboxaldehyde (56.0 mg, $0.5 \mathrm{mmol})$, 2-phenylpyridine 2 (31.1 mg, $0.2 \mathrm{mmol})$, PQ (4.2 mg, $0.02 \mathrm{mmol})$, $\mathrm{Pd}(\mathrm{OAc})_{2}(8.9 \mathrm{mg}, 0.04 \mathrm{mmol}), \mathrm{Ag}_{2} \mathrm{O}(92.4 \mathrm{mg}, 0.4 \mathrm{mmol})$ and $\mathrm{CH}_{3} \mathrm{CN}(4 \mathrm{~mL})$. Time of reaction: 12 hours. The crude mixture was purified by silica gel chromatography (ethyl acetate/petroleum ether 1:6) to afford the product $\mathbf{2 5}$ as a colorless oil (39.8 $\mathrm{mg}$, $75 \%$ yield). ${ }^{1} \mathrm{H}$ NMR (600 MHz, $\left.\mathrm{CDCl}_{3}\right) \delta 8.64(\mathrm{~d}, J=4.2 \mathrm{~Hz}, 1 \mathrm{H}), 7.76(\mathrm{~m}, 1 \mathrm{H}), 7.70$ $-7.65(\mathrm{~m}, 1 \mathrm{H}), 7.59$ (d, J=7.9 Hz, 1H), 7.50 (m, 1H), 7.44 (m, 1H), 7.40 (dd, J=7.6, $1.2 \mathrm{~Hz}, 1 \mathrm{H}), 7.24(\mathrm{~m}, 1 \mathrm{H}), 2.22(\mathrm{~m}, 1 \mathrm{H}), 1.77-1.70(\mathrm{~m}, 2 \mathrm{H}), 1.67(\mathrm{~m}, 2 \mathrm{H}), 1.59-1.51$ $(\mathrm{m}, 1 \mathrm{H}), 1.38(\mathrm{~m}, 2 \mathrm{H}), 1.13(\mathrm{~m}, 1 \mathrm{H}), 0.98(\mathrm{~m}, 2 \mathrm{H}) .{ }^{13} \mathrm{C} \mathrm{NMR}\left(150 \mathrm{MHz}, \mathrm{CDCl}_{3}\right) \delta$ $210.8,157.5,149.5,141.5,138.3,136.8,129.9,128.9,128.7,128.2,122.5,122.3,50.9$, $29.3,26.0,25.9$.

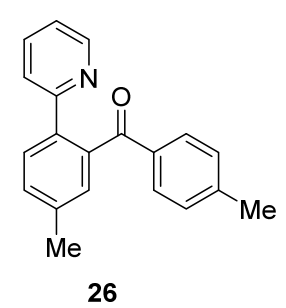

\section{(5-methyl-2-(pyridin-2-yl)phenyl)(p-tolyl)methanone (26)}

Prepared according to the general procedure using 4-methylbenzaldehyde $(60.0 \mathrm{mg}, 0.5$ mmol), 2-(4-Tolyl)pyridine (34.2 mg, $0.2 \mathrm{mmol}$ ), PQ (4.2 mg, $0.02 \mathrm{mmol}), \operatorname{Pd}(\mathrm{OAc}) 2$ (8.9 mg, $0.04 \mathrm{mmol}), \mathrm{Ag}_{2} \mathrm{O}(92.4 \mathrm{mg}, 0.4 \mathrm{mmol})$ and $\mathrm{CH}_{3} \mathrm{CN}$ (4 mL). Time of reaction: 
12 hours. The crude mixture was purified by silica gel chromatography (ethyl acetate/petroleum ether 1:6) to afford the product as a colorless oil (37.4 mg, 65\% yield). ${ }^{1} \mathrm{H}$ NMR $\left(600 \mathrm{MHz}, \mathrm{CDCl}_{3}\right) \delta 8.36(\mathrm{~d}, J=4.3 \mathrm{~Hz}, 1 \mathrm{H}), 7.66(\mathrm{~d}, J=7.9 \mathrm{~Hz}, 1 \mathrm{H}), 7.59$ (d, $J=8.1 \mathrm{~Hz}, 2 \mathrm{H}), 7.52$ (m, 1H), 7.44 (d, $J=7.9 \mathrm{~Hz}, 1 \mathrm{H}), 7.39$ (d, $J=7.8 \mathrm{~Hz}, 1 \mathrm{H})$, $7.31(\mathrm{~s}, 1 \mathrm{H}), 7.06(\mathrm{~d}, J=7.9 \mathrm{~Hz}, 2 \mathrm{H}), 6.98(\mathrm{~m}, 1 \mathrm{H}), 2.43(\mathrm{~s}, 3 \mathrm{H}), 2.30(\mathrm{~s}, 3 \mathrm{H}) .{ }^{13} \mathrm{C} \mathrm{NMR}$ $\left(150 \mathrm{MHz}, \mathrm{CDCl}_{3}\right) \delta 198.3,157.0,149.1,143.1,139.8,138.6,136.9,136.3,135.5$, 130.8, 129.8, 129.6, 128.9, 128.8, 122.7, 121.7, 21.7, 21.3. HRMS (ESI) m/z: [M + H] calcd for $\mathrm{C}_{20} \mathrm{H}_{18} \mathrm{NO} 288.1383$; found 288.1384 .

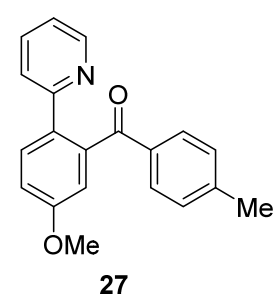

\section{[5-Methoxy-2-(2-pyridinyl)phenyl](4-methylphenyl)methanone (27)}

Prepared according to the general procedure using 4-methylbenzaldehyde ( $60.0 \mathrm{mg}, 0.5$ mmol), 2-(4-Methoxyphenyl)pyridine (37.2 mg, $0.2 \mathrm{mmol})$, PQ (4.2 mg, $0.02 \mathrm{mmol}$ ), $\mathrm{Pd}(\mathrm{OAc})_{2}(8.9 \mathrm{mg}, 0.04 \mathrm{mmol}), \mathrm{Ag}_{2} \mathrm{O}(92.4 \mathrm{mg}, 0.4 \mathrm{mmol})$ and $\mathrm{CH}_{3} \mathrm{CN}(4 \mathrm{~mL})$. Time of reaction: 12 hours. The crude mixture was purified by silica gel chromatography (ethyl acetate/petroleum ether 1:4) to afford the product as a colorless oil (53.2 mg, 88\% yield). ${ }^{1} \mathrm{H}$ NMR (600 MHz, $\left.\mathrm{CDCl}_{3}\right) \delta 8.32(\mathrm{~d}, J=4.2 \mathrm{~Hz}, 1 \mathrm{H}), 7.71(\mathrm{~d}, J=8.6 \mathrm{~Hz}, 1 \mathrm{H})$, $7.60(\mathrm{~d}, J=8.0 \mathrm{~Hz}, 2 \mathrm{H}), 7.50(\mathrm{~m}, 1 \mathrm{H}), 7.42$ (d, $J=7.9 \mathrm{~Hz}, 1 \mathrm{H}), 7.10$ (dd, $J=8.6,2.5$ $\mathrm{Hz}, 1 \mathrm{H}), 7.05$ (d, J=7.9 Hz, 2H), 7.01 (d, $J=2.4 \mathrm{~Hz}, 1 \mathrm{H}), 6.94$ (m, 1H), 3.85 (s, 3H), 2.29 (s, 3H). ${ }^{13} \mathrm{C}$ NMR (150 MHz, $\left.\mathrm{CDCl}_{3}\right) \delta$ 197.7, 159.9, 156.5, 149.0, 143.2, 141.1, 136.2, 135.3, 132.0, 130.1, 129.7, 128.9, 122.2, 121.4, 116.0, 113.9, 55.6, 21.6. HRMS (ESI) $\mathrm{m} / \mathrm{z}:[\mathrm{M}+\mathrm{H}]^{+}$calcd for $\mathrm{C}_{20} \mathrm{H}_{18} \mathrm{NO}_{2}$ 304.1332; found 304.1333.

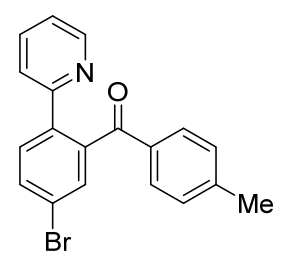

28 


\section{(5-bromo-2-(pyridin-2-yl)phenyl)(p-tolyl)methanone (28)}

Prepared according to the general procedure using 4-methylbenzaldehyde (60.0 mg, 0.5 mmol), 2-(4-Bromophenyl)pyridine (46.8 mg, $0.2 \mathrm{mmol})$, PQ (4.2 mg, $0.02 \mathrm{mmol})$, $\mathrm{Pd}(\mathrm{OAc})_{2}(8.9 \mathrm{mg}, 0.04 \mathrm{mmol}), \mathrm{Ag}_{2} \mathrm{O}$ (92.4 mg, $\left.0.4 \mathrm{mmol}\right)$ and $\mathrm{CH}_{3} \mathrm{CN}$ (4 mL). Time of reaction: 12 hours. The crude mixture was purified by silica gel chromatography (ethyl acetate/petroleum ether 1:10) to afford the product as a colorless oil (38.2 mg, 54\% yield). ${ }^{1} \mathrm{H}$ NMR (600 MHz, $\left.\mathrm{CDCl}_{3}\right) \delta 8.36(\mathrm{~d}, J=4.2 \mathrm{~Hz}, 1 \mathrm{H}), 7.71(\mathrm{~m}, 1 \mathrm{H}), 7.63$ (m, 2H), $7.60-7.54(\mathrm{~m}, 3 \mathrm{H}), 7.46$ (d, J=7.9 Hz, 1H), 7.09 (d, J=8.0 Hz, 2H), 7.03 (m, 1H), $2.32(\mathrm{~s}, 3 \mathrm{H}) .{ }^{13} \mathrm{C} \mathrm{NMR}\left(150 \mathrm{MHz}, \mathrm{CDCl}_{3}\right) \delta 196.3,155.9,149.3,143.7,141.5$, $138.5,136.5,134.9,133.1,131.8,130.5,129.8,129.1,122.9,122.6,122.3,21.7$. HRMS (ESI) $\mathrm{m} / \mathrm{z}:[\mathrm{M}+\mathrm{H}]^{+}$calcd for $\mathrm{C}_{19} \mathrm{H}_{15} \mathrm{BrNO} 352.0332$; found 352.0333 .

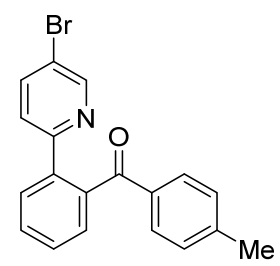

29

\section{(2-(5-bromopyridin-2-yl)phenyl)(p-tolyl)methanone (29)}

Prepared according to the general procedure using 4-methylbenzaldehyde (60.0 mg, 0.5 mmol), 5-Bromo-2-phenylpyridine (46.8 mg, $0.2 \mathrm{mmol})$, PQ (4.2 mg, $0.02 \mathrm{mmol}$ ), $\mathrm{Pd}(\mathrm{OAc})_{2}(8.9 \mathrm{mg}, 0.04 \mathrm{mmol}), \mathrm{Ag}_{2} \mathrm{O}$ (92.4 mg, $\left.0.4 \mathrm{mmol}\right)$ and $\mathrm{CH}_{3} \mathrm{CN}$ (4 mL). Time of reaction: 12 hours. The crude mixture was purified by silica gel chromatography (ethyl acetate/petroleum ether 1:10) to afford the product as a colorless oil (36.6 mg, $52 \%$ yield). ${ }^{1} \mathrm{H}$ NMR $\left(600 \mathrm{MHz}, \mathrm{CDCl}_{3}\right) \delta 8.44(\mathrm{~d}, J=2.2 \mathrm{~Hz}, 1 \mathrm{H}), 7.77-7.68(\mathrm{~m}$, 2H), $7.62(\mathrm{~d}, J=8.1 \mathrm{~Hz}, 2 \mathrm{H}), 7.58(\mathrm{~m}, 1 \mathrm{H}), 7.53-7.47(\mathrm{~m}, 2 \mathrm{H}), 7.39$ (d, $J=8.4 \mathrm{~Hz}$, 1H), $7.12(\mathrm{~d}, J=8.1 \mathrm{~Hz}, 2 \mathrm{H}), 2.35$ (s, 3H). ${ }^{13} \mathrm{C} \mathrm{NMR}\left(150 \mathrm{MHz}, \mathrm{CDCl}_{3}\right) \delta$ 197.8, 155.7, $150.3,143.7,139.8,139.0,138.5,135.2,130.2,130.0,129.1,129.0,128.8,124.0,119.5$, 21.8. HRMS (ESI) m/z: $[\mathrm{M}+\mathrm{H}]^{+}$calcd for $\mathrm{C}_{19} \mathrm{H}_{15} \mathrm{BrNO} 352.0332$; found 352.0336, $[\mathrm{M}+\mathrm{Na}]^{+}$calcd for $\mathrm{C}_{19} \mathrm{H}_{14} \mathrm{BrNONa} 374.0151$; found 374.0156. 


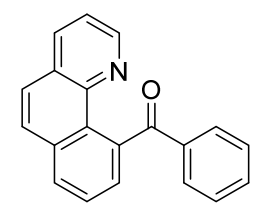

30

\section{Benzo[h]quinolin-10-yl(phenyl)methanone (30) ${ }^{1}$}

Prepared according to the general procedure using benzaldehyde $(83.7 \mathrm{mg}, 0.5 \mathrm{mmol})$, 7,8-benzoquinoline (35.8 mg, $0.2 \mathrm{mmol}), \mathrm{PQ}$ (4.2 mg, $0.02 \mathrm{mmol}), \mathrm{Pd}(\mathrm{OAc})_{2}(8.9 \mathrm{mg}$, $0.04 \mathrm{mmol}), \mathrm{Ag}_{2} \mathrm{O}(92.4 \mathrm{mg}, 0.4 \mathrm{mmol})$ and $\mathrm{CH}_{3} \mathrm{CN}(4 \mathrm{~mL})$. Time of reaction: 12 hours. The crude mixture was purified by silica gel chromatography (ethyl acetate/petroleum ether 1:20) to afford the product 30as a colorless oil (22.6 mg, 40\% yield). ${ }^{1} \mathrm{H}$ NMR $\left(600 \mathrm{MHz}, \mathrm{CDCl}_{3}\right) \delta 8.50(\mathrm{dd}, J=4.2,1.3 \mathrm{~Hz}, 1 \mathrm{H}), 8.12-8.07(\mathrm{dd}, J=8.0,1.2 \mathrm{~Hz}$, 1H ), 8.05 (d, $J=7.9 \mathrm{~Hz}, 1 \mathrm{H}), 7.90$ (d, $J=8.8 \mathrm{~Hz}, 1 \mathrm{H}), 7.77$ (m, 4H), 7.63 (d, $J=7.1$ $\mathrm{Hz}, 1 \mathrm{H}), 7.41(\mathrm{~m}, 1 \mathrm{H}), 7.35-7.27(\mathrm{~m}, 3 \mathrm{H}) .{ }^{13} \mathrm{C} \mathrm{NMR}\left(150 \mathrm{MHz}, \mathrm{CDCl}_{3}\right) \delta$ 198.7, 147.2, $144.8,139.4,139.1,135.4,134.0,131.8,129.4,129.1,128.8,128.2,127.9,127.9,127.1$, $126.6,126.2,121.8$.

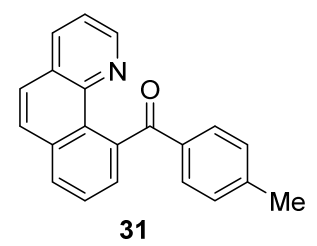

\section{Benzo[h]quinolin-10-yl(p-tolyl)methanone (31)}

Prepared according to the general procedure using 4-methylbenzaldehyde (60.0 mg, 0.5 mmol), 7,8-benzoquinoline (35.8 mg, $0.2 \mathrm{mmol})$, PQ (4.2 mg, $0.02 \mathrm{mmol}), \mathrm{Pd}(\mathrm{OAc})_{2}$ ( $8.9 \mathrm{mg}, 0.04 \mathrm{mmol}), \mathrm{Ag}_{2} \mathrm{O}$ (92.4 mg, $0.4 \mathrm{mmol}$ ) and $\mathrm{CH}_{3} \mathrm{CN}$ (4 mL). Time of reaction: 12 hours. The crude mixture was purified by silica gel chromatography (ethyl acetate/petroleum ether 1:20) to afford the product $\mathbf{3 1}$ as a colorless oil $(25.5 \mathrm{mg}, 43 \%$ yield). ${ }^{1} \mathrm{H} \mathrm{NMR}\left(600 \mathrm{MHz}, \mathrm{CDCl}_{3}\right) \delta 8.53(\mathrm{dd}, J=4.3,1.5 \mathrm{~Hz}, 1 \mathrm{H}), 8.10$ (dd, $J=8.0$, $1.4 \mathrm{~Hz}, 1 \mathrm{H}), 8.03(\mathrm{~d}, J=7.8 \mathrm{~Hz}, 1 \mathrm{H}), 7.89$ (d, $J=8.8 \mathrm{~Hz}, 1 \mathrm{H}), 7.80-7.72(\mathrm{~m}, 2 \mathrm{H})$, $7.65(\mathrm{~d}, J=8.1 \mathrm{~Hz}, 2 \mathrm{H}), 7.60(\mathrm{~d}, J=6.7 \mathrm{~Hz}, 1 \mathrm{H}), 7.33(\mathrm{dd}, J=8.0,4.4 \mathrm{~Hz}, 1 \mathrm{H}), 7.10$ $(\mathrm{d}, J=8.0 \mathrm{~Hz}, 2 \mathrm{H}), 2.33$ (s, 3H). ${ }^{13} \mathrm{C} \mathrm{NMR}\left(150 \mathrm{MHz}, \mathrm{CDCl}_{3}\right) \delta$ 198.5, 147.3, 145.0, 
$142.4,139.4,136.9,135.4,134.0,129.3,129.1,129.0,128.9,127.9,127.8,127.1,126.5$, 126.2, 121.8, 21.7. HRMS (ESI) m/z: [M + H] calcd for $\mathrm{C}_{21} \mathrm{H}_{16} \mathrm{NO}$ 298.1226; found 298.1224 .

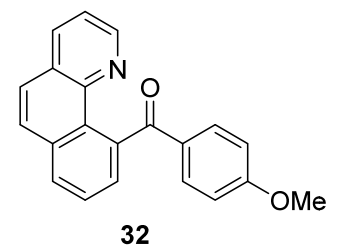

\section{Benzo[h]quinolin-10-yl(4-methoxyphenyl)methanone (32)}

Prepared according to the general procedure using 4-methoxybenzaldehyde $(68.2 \mathrm{mg}$, $0.5 \mathrm{mmol}$ ), 7,8-benzoquinoline (35.8 $\mathrm{mg}, 0.2 \mathrm{mmol})$, PQ (4.2 $\mathrm{mg}, 0.02 \mathrm{mmol}$ ), $\mathrm{Pd}(\mathrm{OAc})_{2}(8.9 \mathrm{mg}, 0.04 \mathrm{mmol}), \mathrm{Ag}_{2} \mathrm{O}$ (92.4 mg, $\left.0.4 \mathrm{mmol}\right)$ and $\mathrm{CH}_{3} \mathrm{CN}$ (4 mL). Time of reaction: 12 hours. The crude mixture was purified by silica gel chromatography (ethyl acetate/petroleum ether 1:20) to afford the product 32 as a colorless oil (28.2 $\mathrm{mg}$, $45 \%$ yield). ${ }^{1} \mathrm{H} \mathrm{NMR}\left(600 \mathrm{MHz}, \mathrm{CDCl}_{3}\right) \delta 8.54(\mathrm{dd}, J=4.3,1.6 \mathrm{~Hz}, 1 \mathrm{H}), 8.10(\mathrm{dd}, J=$ 8.0, 1.4 Hz, 1H), 8.02 (d, J=7.9 Hz, 1H), 7.89 (d, J=8.8 Hz, 1H), 7.75 (m, 4H), 7.59 $(\mathrm{d}, J=7.1 \mathrm{~Hz}, 1 \mathrm{H}), 7.34(\mathrm{dd}, J=8.0,4.4 \mathrm{~Hz}, 1 \mathrm{H}), 6.79(\mathrm{~d}, J=9.0 \mathrm{~Hz}, 2 \mathrm{H}), 3.78(\mathrm{~s}$, $3 \mathrm{H}) .{ }^{13} \mathrm{C} \mathrm{NMR}\left(150 \mathrm{MHz}, \mathrm{CDCl}_{3}\right) \delta 197.8,162.7,147.4,145.1,139.5,135.4,134.1$, $132.5,131.1,129.3,129.0,128.0,127.8,127.1,126.5,126.2,121.7,113.5,55.4$.

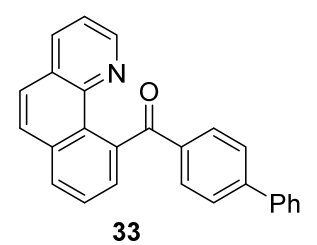

\section{[1,1'-Biphenyl]-4-yl(benzo[h]quinolin-10-yl)methanone (33)}

Prepared according to the general procedure using 4-biphenylcarboxaldehyde $(91.1 \mathrm{mg}$, $0.5 \mathrm{mmol}$ ), 7,8-benzoquinoline (35.8 $\mathrm{mg}, 0.2 \mathrm{mmol})$, PQ (4.2 $\mathrm{mg}, 0.02 \mathrm{mmol}$ ), $\mathrm{Pd}(\mathrm{OAc})_{2}(8.9 \mathrm{mg}, 0.04 \mathrm{mmol}), \mathrm{Ag}_{2} \mathrm{O}$ (92.4 mg, $\left.0.4 \mathrm{mmol}\right)$ and $\mathrm{CH}_{3} \mathrm{CN}$ (4 mL). Time of reaction: 12 hours. The crude mixture was purified by silica gel chromatography (ethyl acetate/petroleum ether 1:15) to afford the product $\mathbf{3 3}$ as a colorless oil (37.3 $\mathrm{mg}$, 
$52 \%$ yield). ${ }^{1} \mathrm{H}$ NMR $\left(600 \mathrm{MHz}, \mathrm{CDCl}_{3}\right) \delta 8.55(\mathrm{~d}, J=3.0 \mathrm{~Hz}, 1 \mathrm{H}), 8.12(\mathrm{~d}, J=7.2 \mathrm{~Hz}$, 1H), $8.07(\mathrm{~d}, J=7.9 \mathrm{~Hz}, 1 \mathrm{H}), 7.92(\mathrm{~d}, J=8.8 \mathrm{~Hz}, 1 \mathrm{H}), 7.81(\mathrm{~m}, 3 \mathrm{H}), 7.76(\mathrm{~d}, J=8.8$ $\mathrm{Hz}, 1 \mathrm{H}), 7.65$ (d, J=7.0 Hz, 1H), 7.55 (m, 4H), 7.41 (m, 2H), 7.35 (dd, J= 7.7, 4.4 Hz, 2H). ${ }^{13} \mathrm{C}$ NMR (150 MHz, $\left.\mathrm{CDCl}_{3}\right) \delta$ 198.3, 147.3, 144.9, 144.5, 140.4, 139.2, 138.3, 135.4, 134.0, 129.4, 129.1, 129.0, 128.0, 127.9, 127.9, 127.3, 127.2, 126.9, 126.6, 126.3, 121.8. HRMS (ESI) m/z: [M+ H] $]^{+}$calcd for $\mathrm{C}_{26} \mathrm{H}_{18} \mathrm{NO} 360.1383$; found 360.1382 .

\section{References:}

1. Jia, X.; Zhang, S.; Wang, W.; Luo, F.; Cheng, J., Palladium-Catalyzed Acylation of $\mathrm{sp}^{2} \mathrm{C}-\mathrm{H}$ bond: Direct Access to Ketones from Aldehydes. Org. Lett. 2009, 11, 31203123.

2. Li, M.; Ge, H., Decarboxylative Acylation of Arenes with $\alpha$-Oxocarboxylic Acids via Palladium-Catalyzed C-H Activation. Org. Lett. 2010, 12, 3464-3467.

3. Zhang, D.; Zhaorigetu, B.; Bao, Y.-S., Supported Palladium Nanoparticles Catalyzed Ortho-Directed $\mathrm{C}-\mathrm{C}$ Coupling Reaction via a $\mathrm{Pd}^{0} / \mathrm{Pd}^{\mathrm{II}} / \mathrm{Pd}^{\mathrm{IV}}$ Catalytic Cycle. J. Phys. Chem. C 2015, 119, 20426-20432.

4. Baslé, O.; Bidange, J.; Shuai, Q.; Li, C.-J., Palladium-Catalyzed Oxidative sp2 CH Bond Acylation with Aldehydes. Adv. Synth. Catal. 2010, 352, 1145-1149.

5. Kochi, T.; Tazawa, A.; Honda, K.; Kakiuchi, F., Ruthenium-catalyzed acylation of arylpyridines with acyl chlorides via ortho-selective $\mathrm{C}-\mathrm{H}$ bond cleavage. Chem. Lett. 2011, 40, 1018-1020. 
NMR Spectral Data

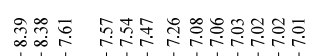

$\bar{i}$

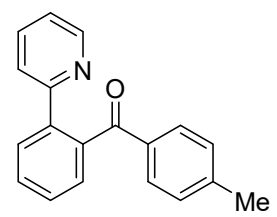

3, $600 \mathrm{MHz}, \mathrm{CDCl}_{3}$

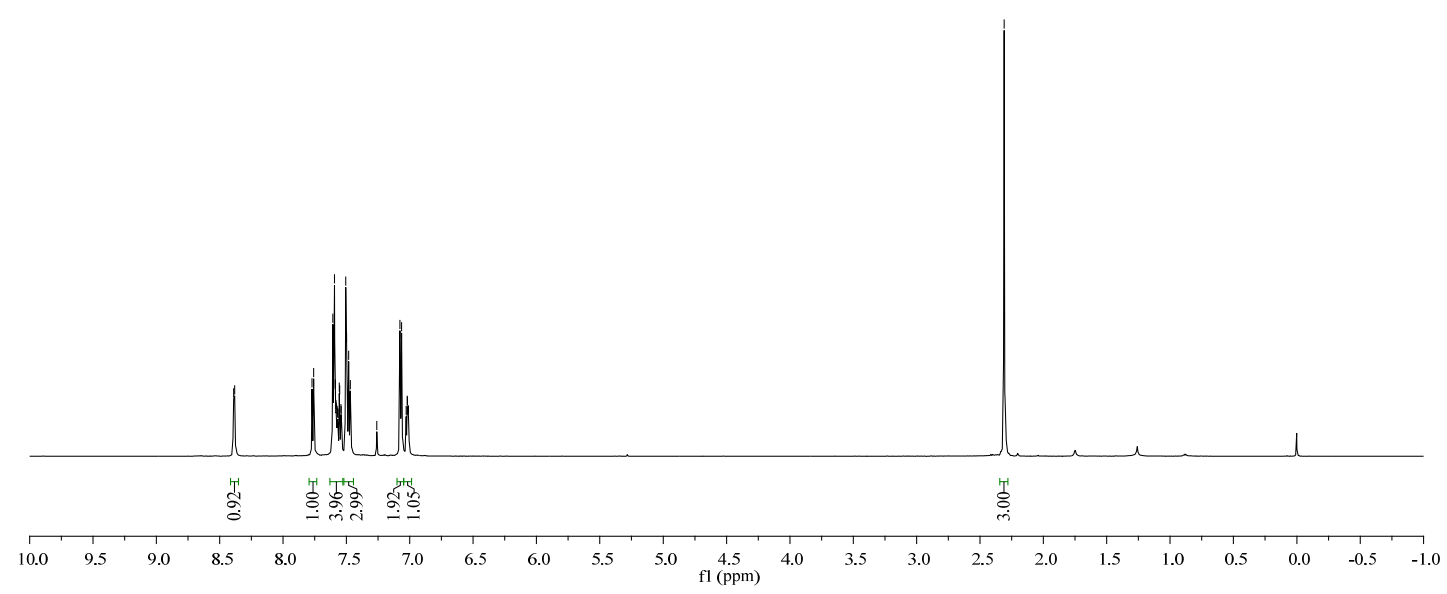

$\frac{\mathfrak{\infty}}{1}$

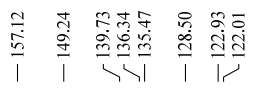

$\underbrace{\frac{3}{2}+\frac{2}{}}$

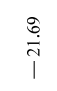

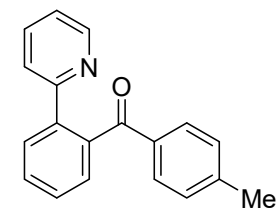

3, $150 \mathrm{MHz}, \mathrm{CDCl}_{3}$

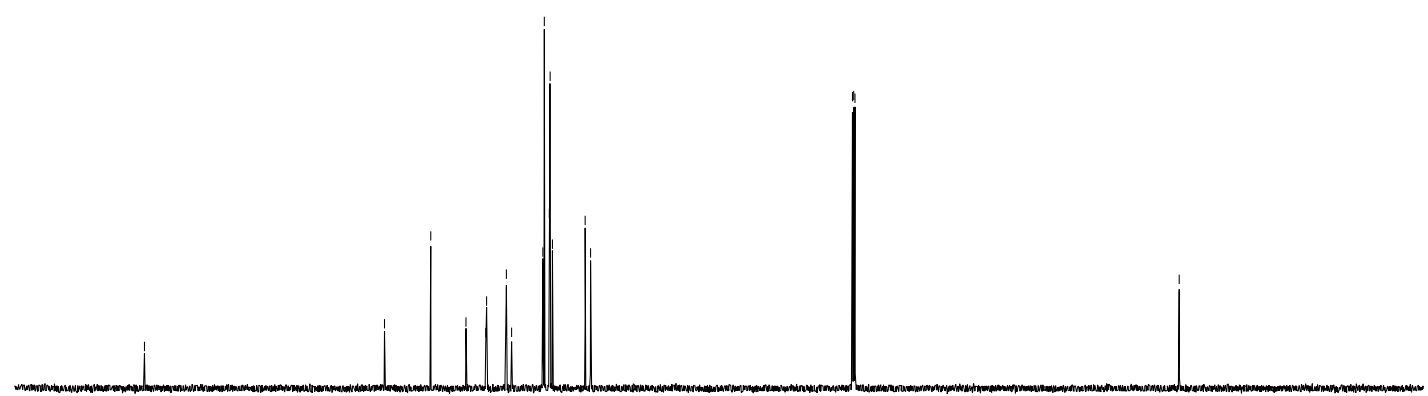

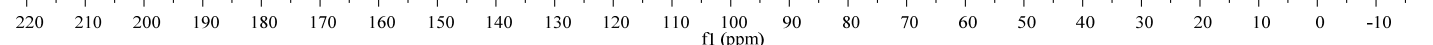




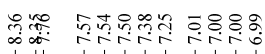

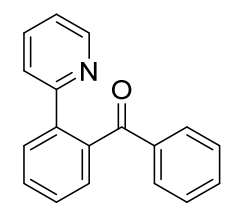

4, $600 \mathrm{MHz}, \mathrm{CDCl}_{3}$
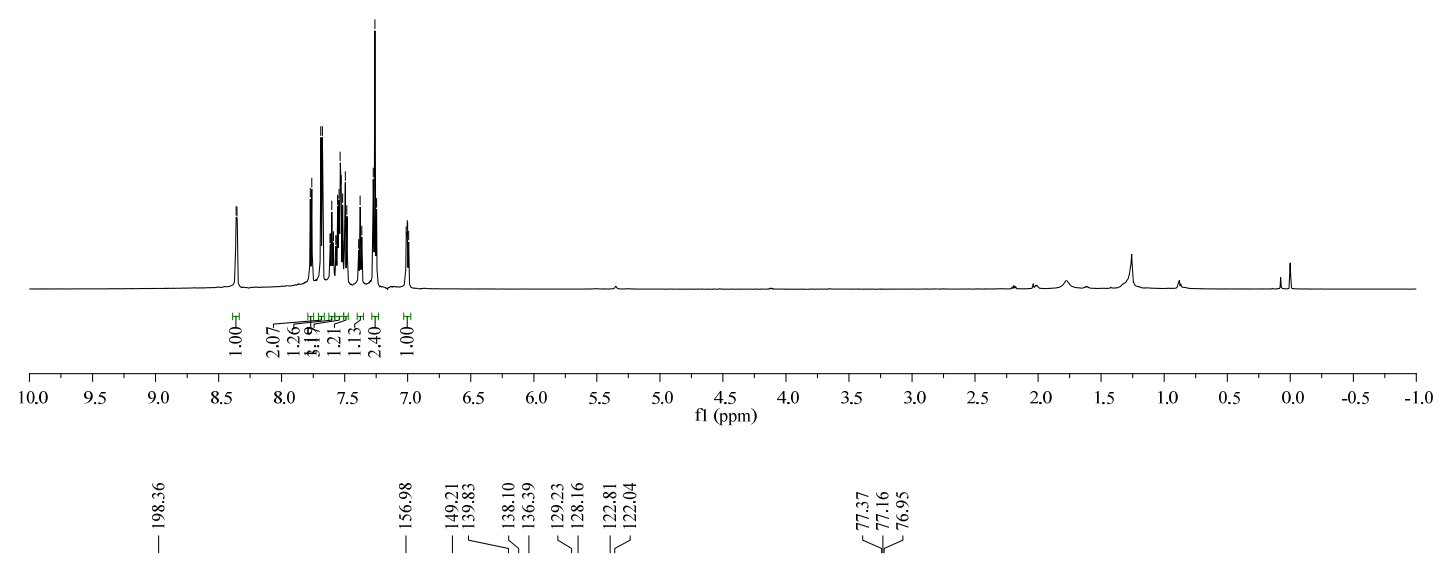

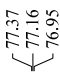

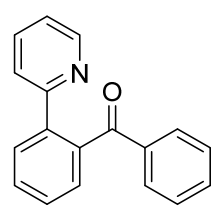

4, $150 \mathrm{MHz}, \mathrm{CDCl}_{3}$

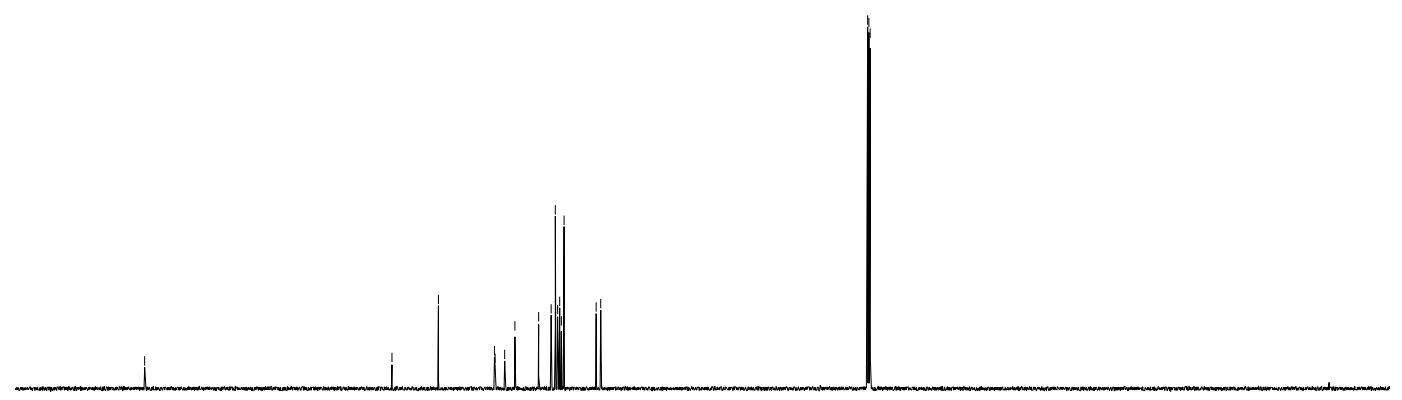

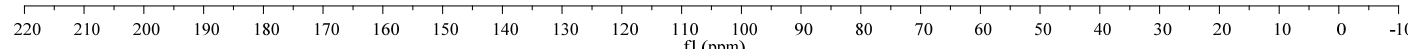




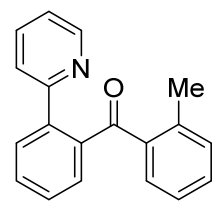

$5,600 \mathrm{MHz}, \mathrm{CDCl}_{3}$
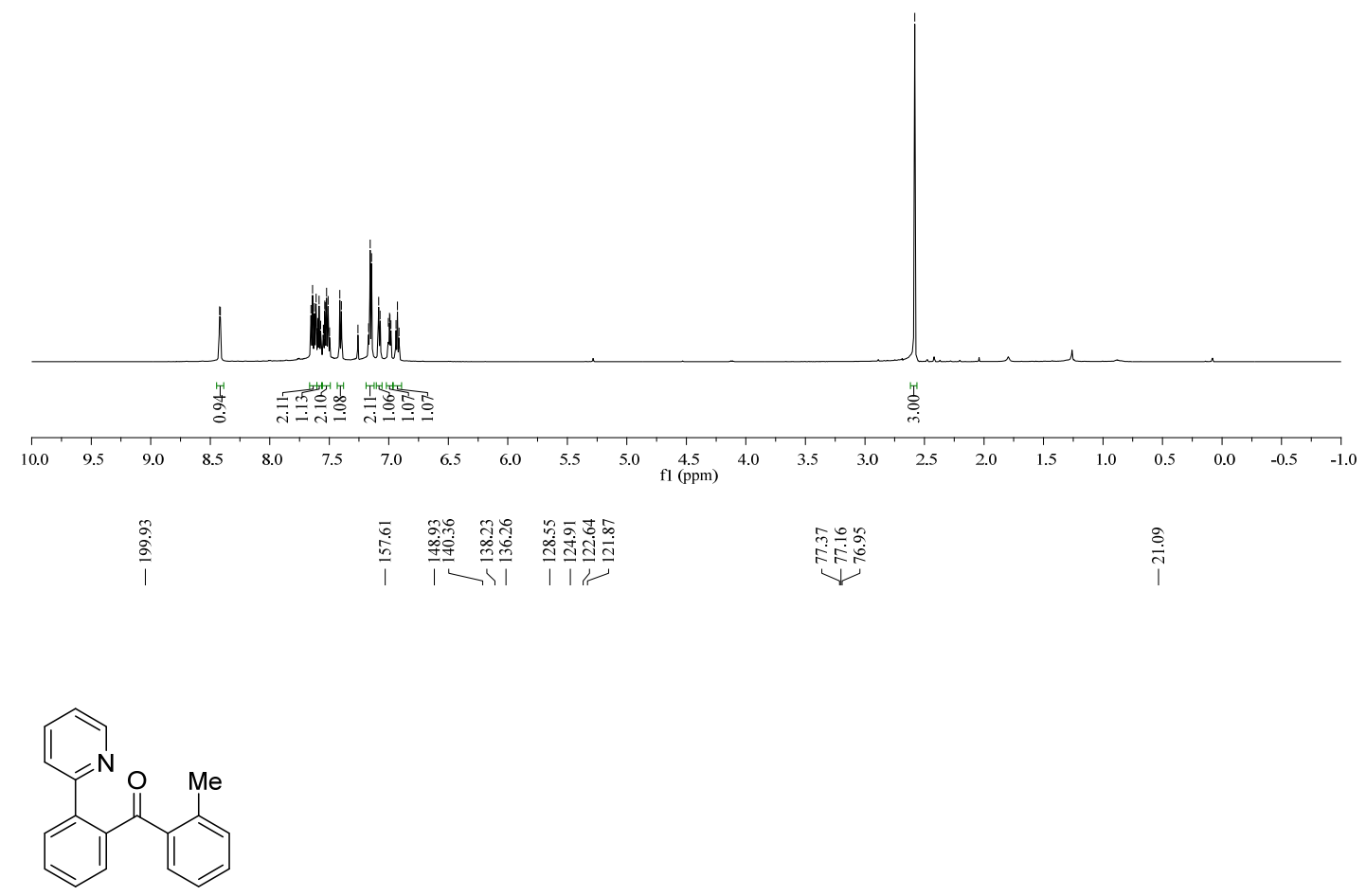

5, $150 \mathrm{MHz}, \mathrm{CDCl}_{3}$

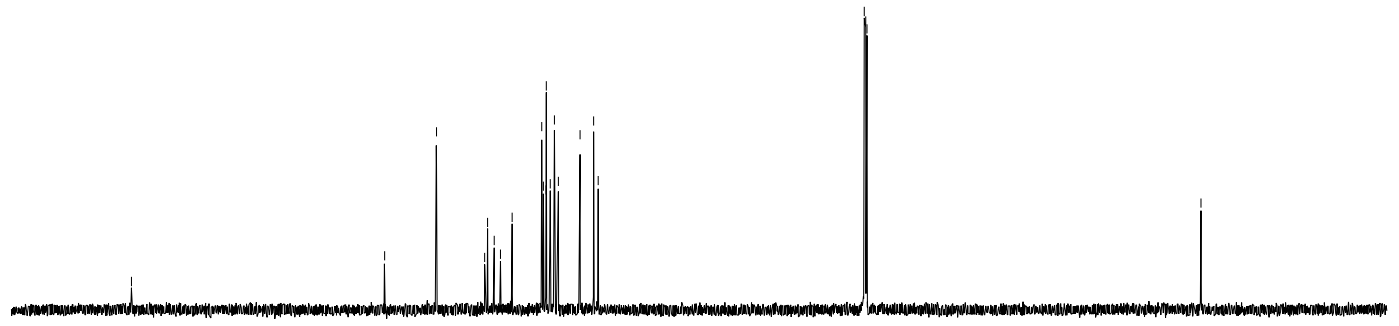

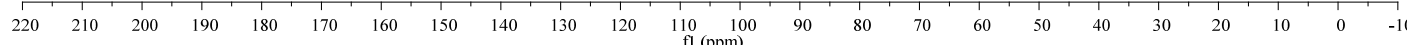




\section{Y}

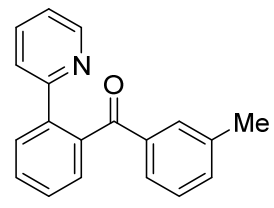

6, $600 \mathrm{MHz}, \mathrm{CDCl}_{3}$
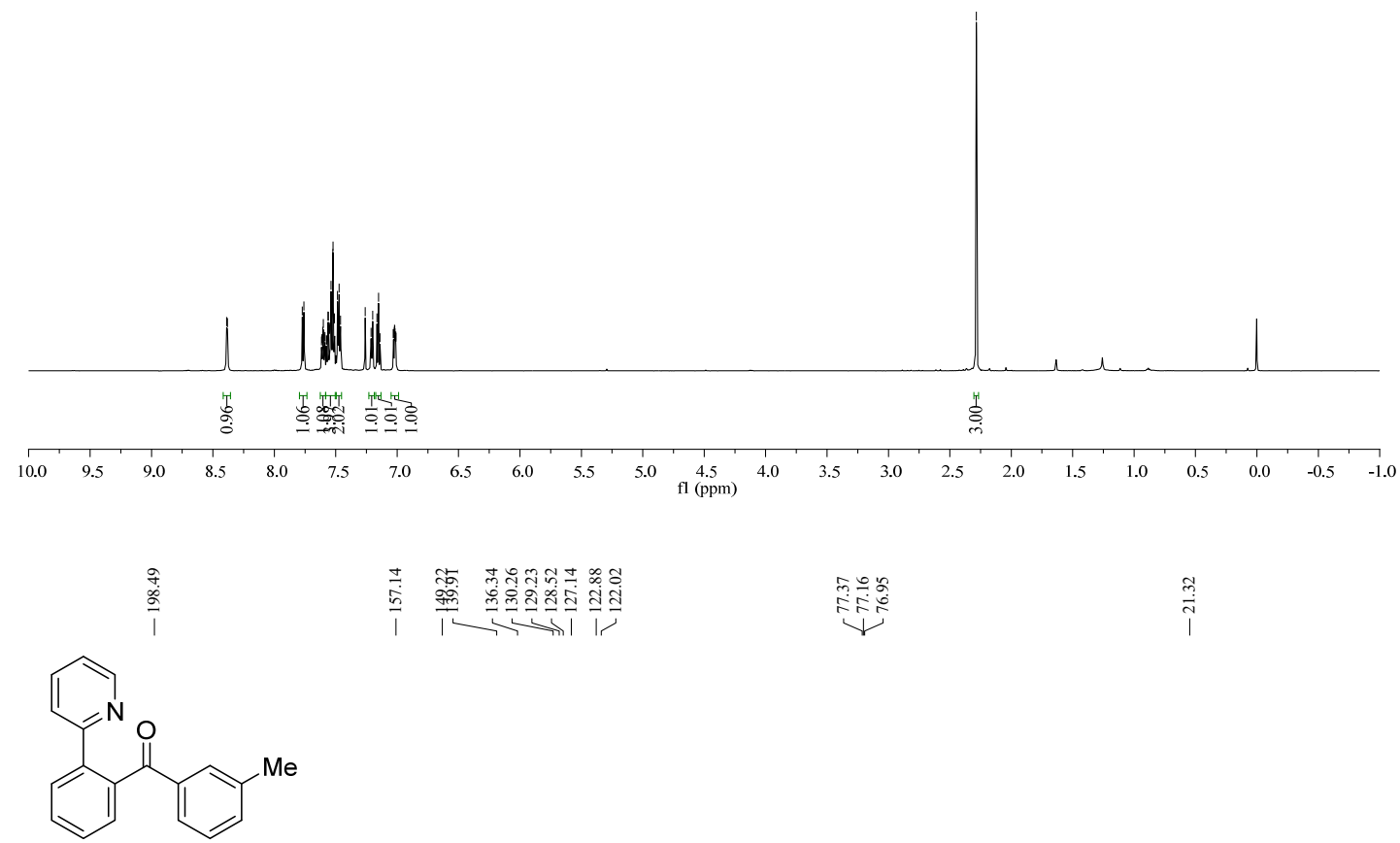

6, $150 \mathrm{MHz}, \mathrm{CDCl}_{3}$

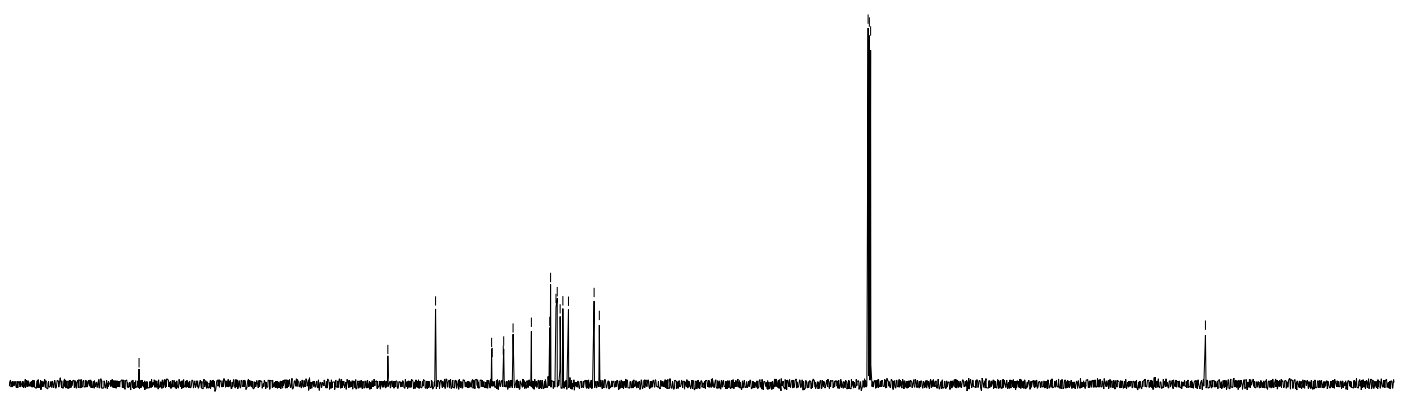

$\begin{array}{lllllllllllllllllllllllllllll}220 & 210 & 200 & 190 & 180 & 170 & 160 & 150 & 140 & 130 & 120 & 110 & 100 & 90 & 80 & 70 & 60 & 50 & 40 & 30 & 20 & 10 & 0 & -10\end{array}$ 


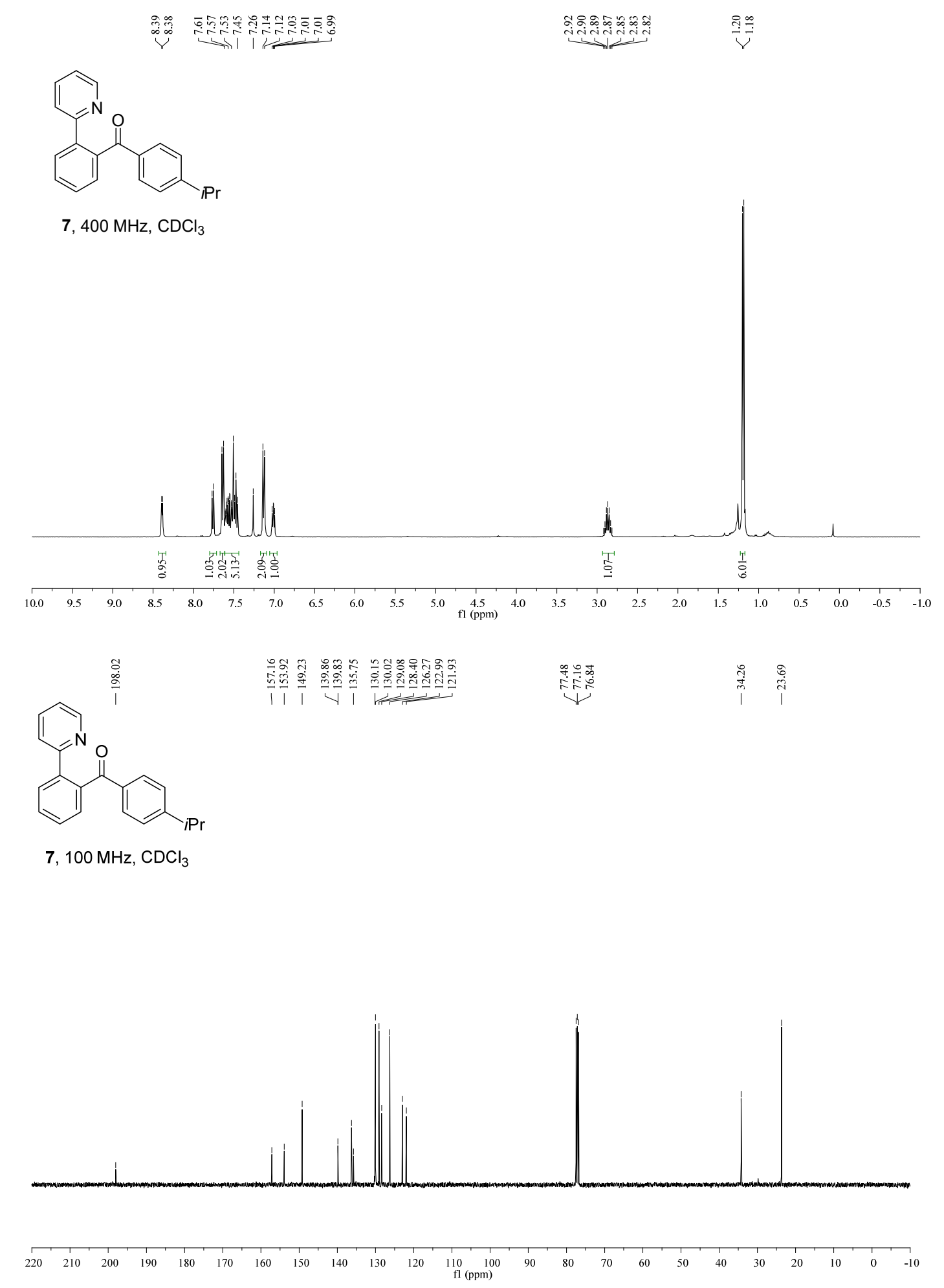




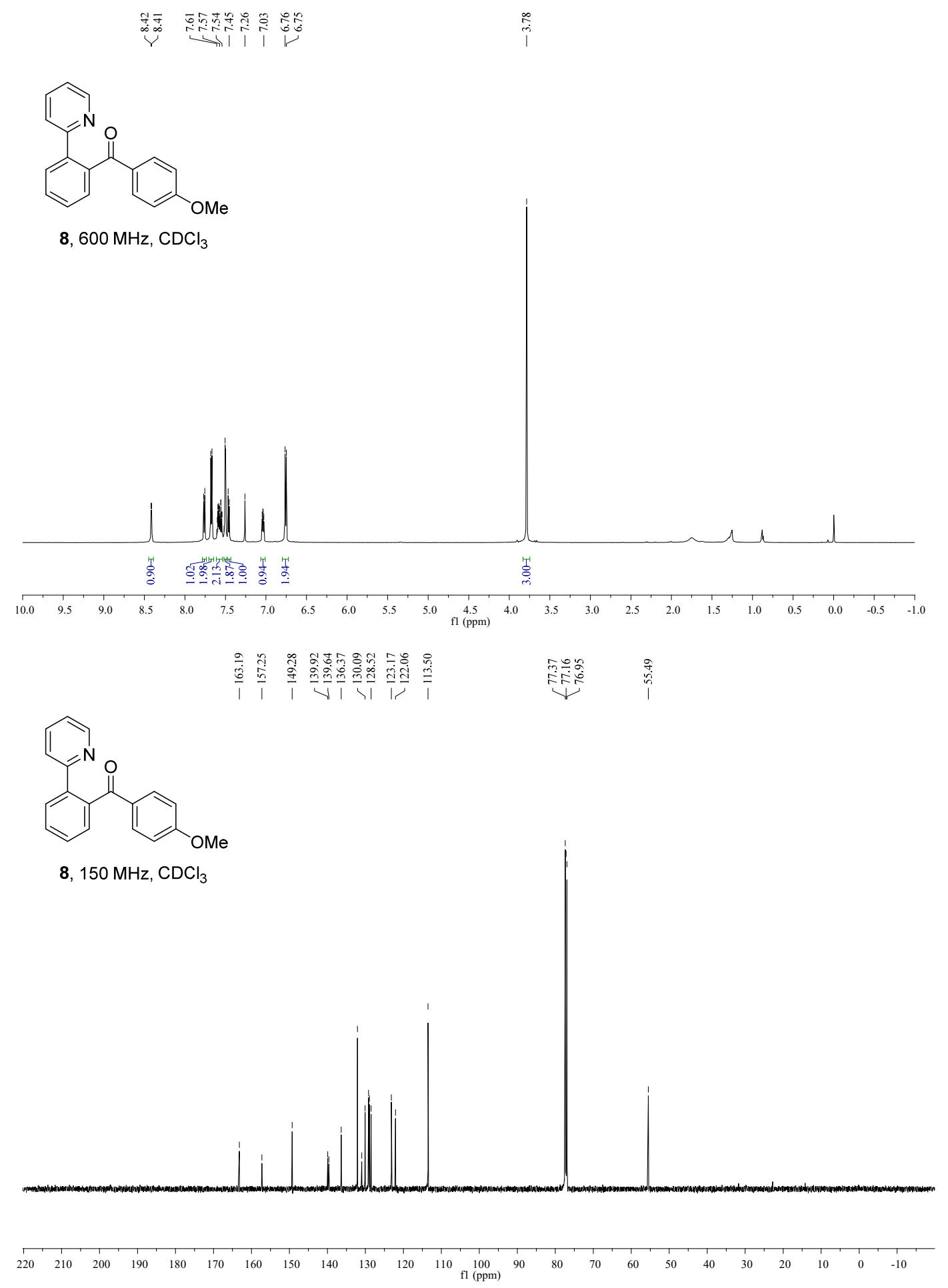




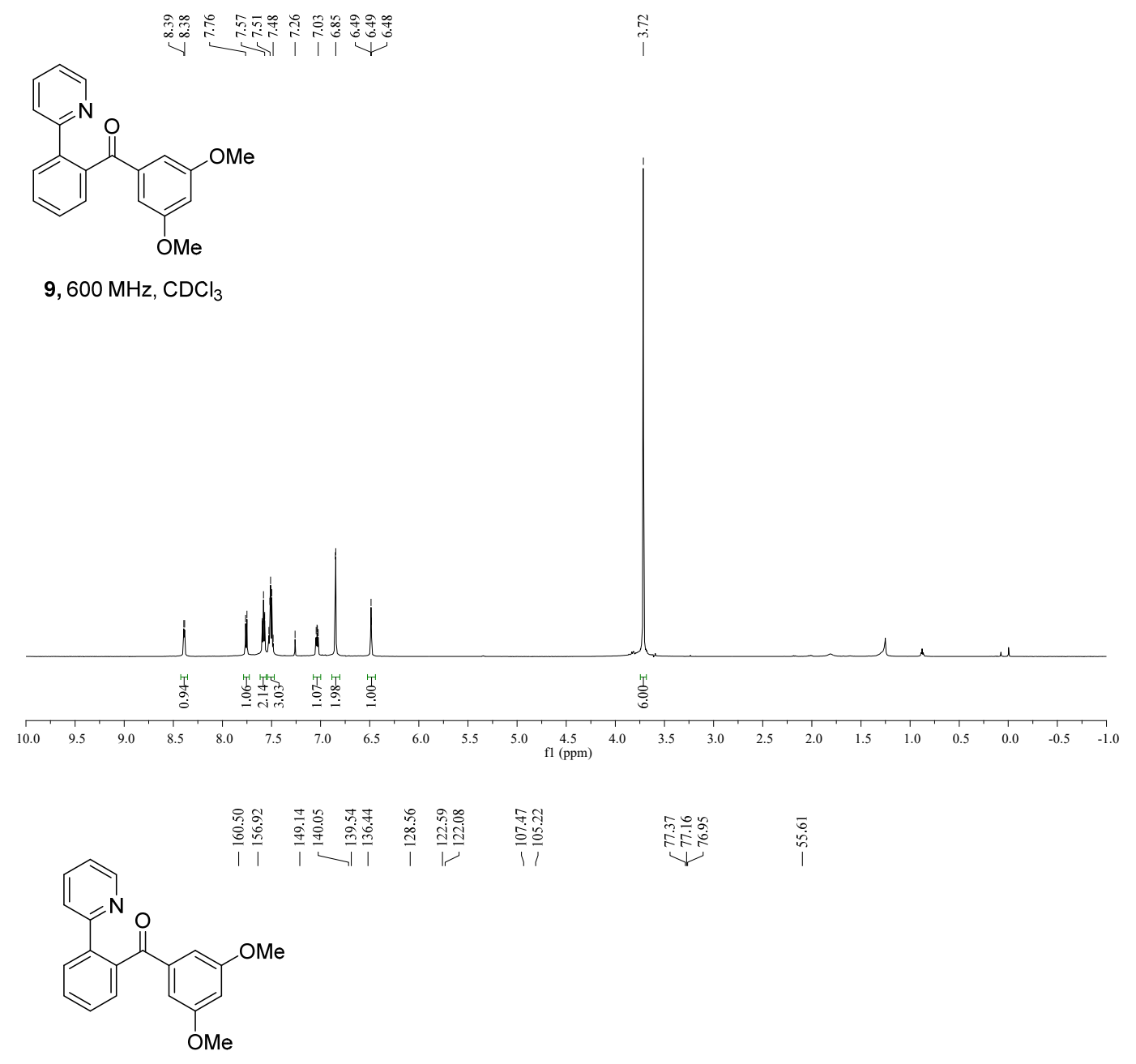

9, $150 \mathrm{MHz}, \mathrm{CDCl}_{3}$

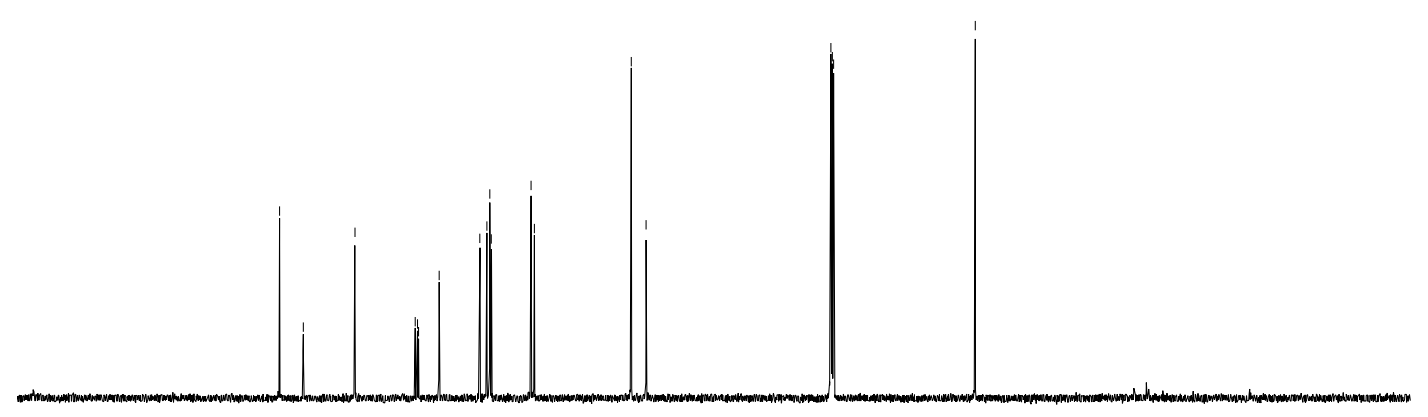

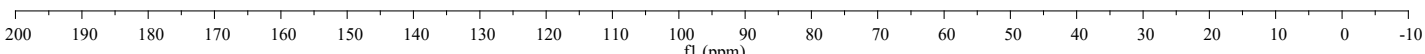




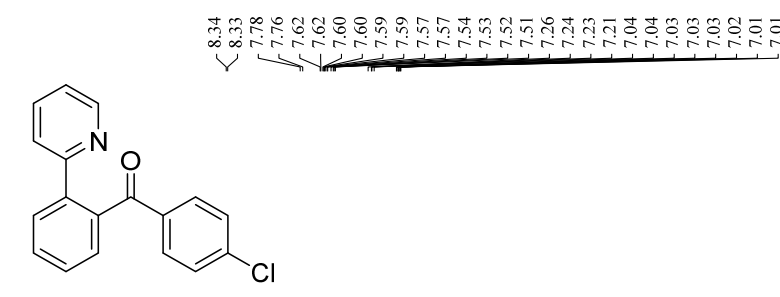

10, $400 \mathrm{MHz}, \mathrm{CDCl}_{3}$

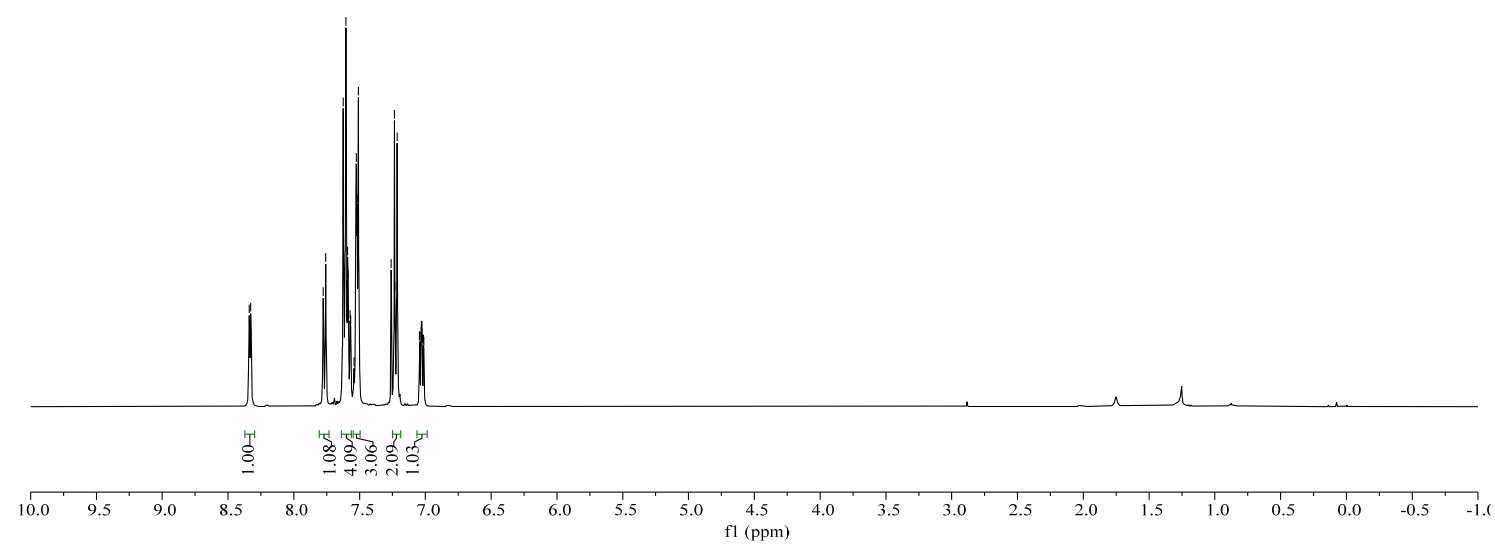

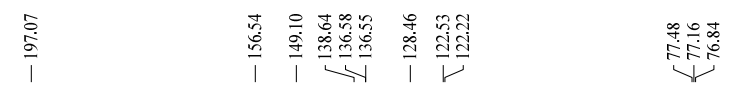

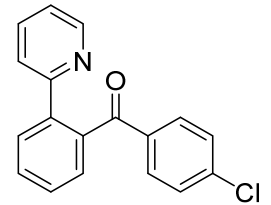

10, $400 \mathrm{MHz}, \mathrm{CDCl}_{3}$

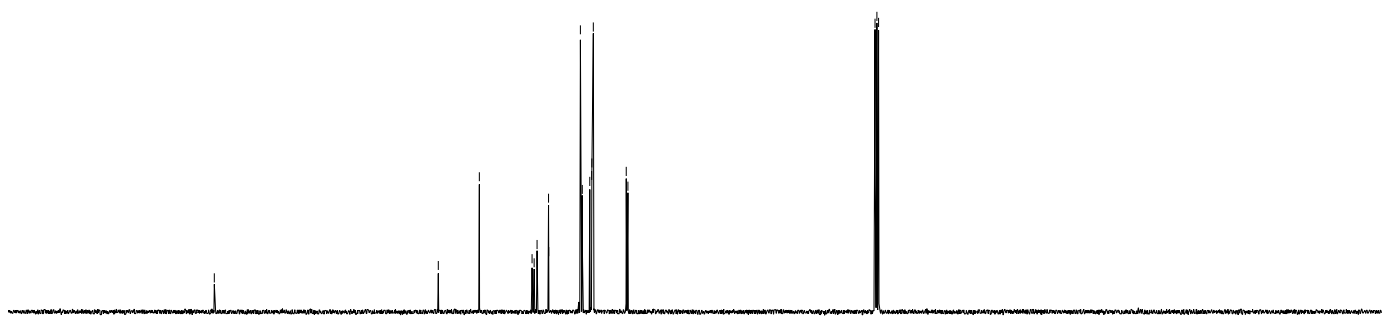

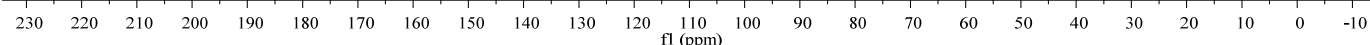




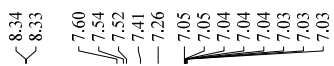

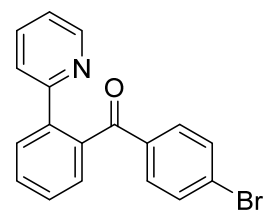

11, $600 \mathrm{MHz}, \mathrm{CDCl}_{3}$

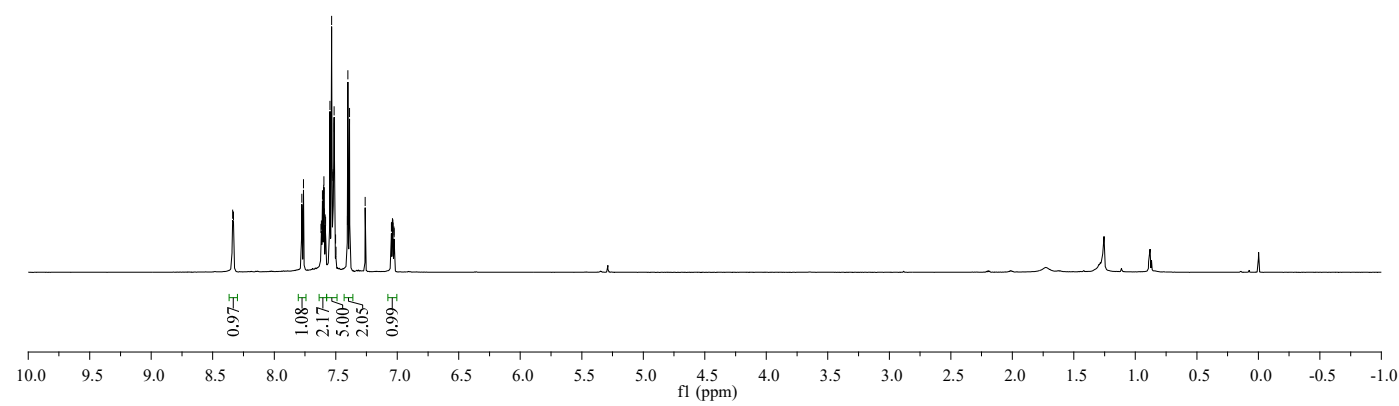

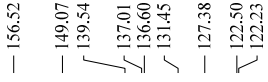

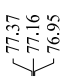

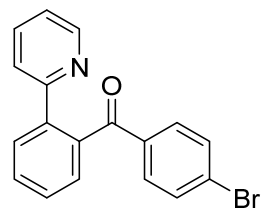

11, $150 \mathrm{MHz}, \mathrm{CDCl}_{3}$

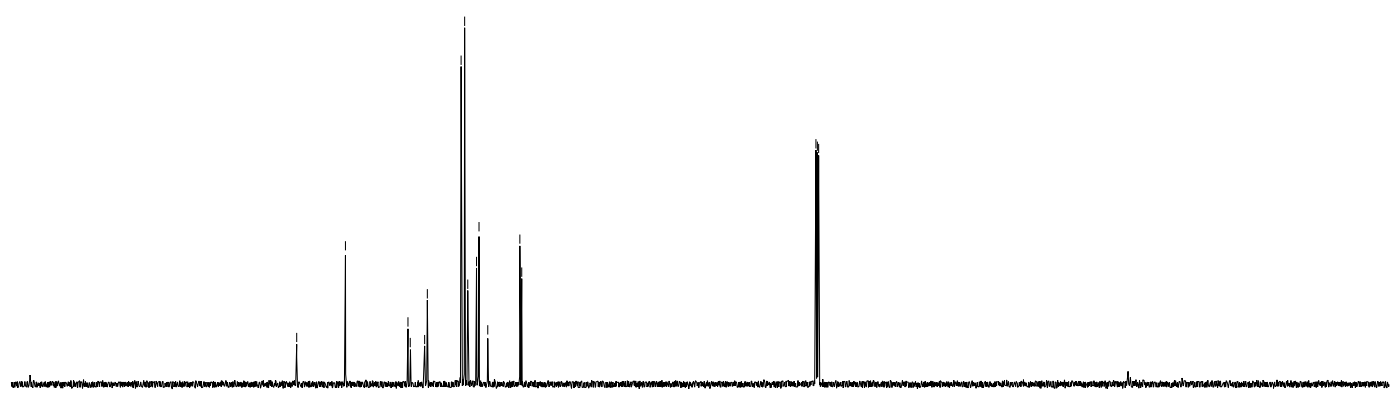

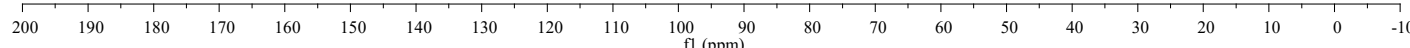




\section{Y)}

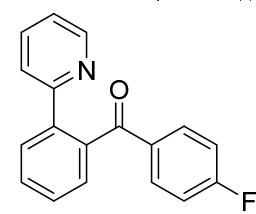

$12,600 \mathrm{MHz}, \mathrm{CDCl}_{3}$

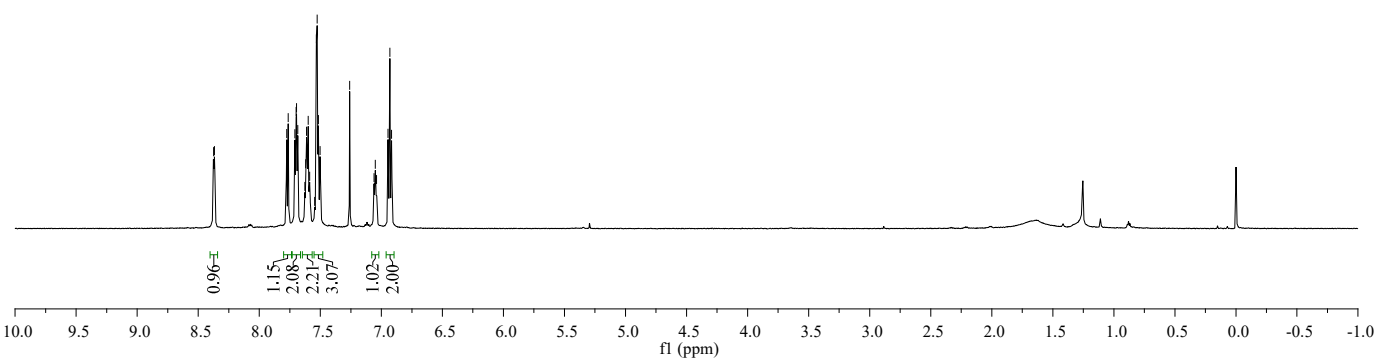

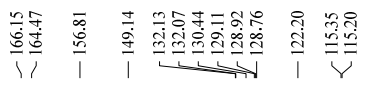

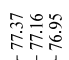

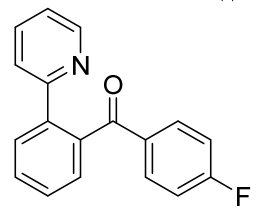

12, $150 \mathrm{MHz}, \mathrm{CDCl}_{3}$

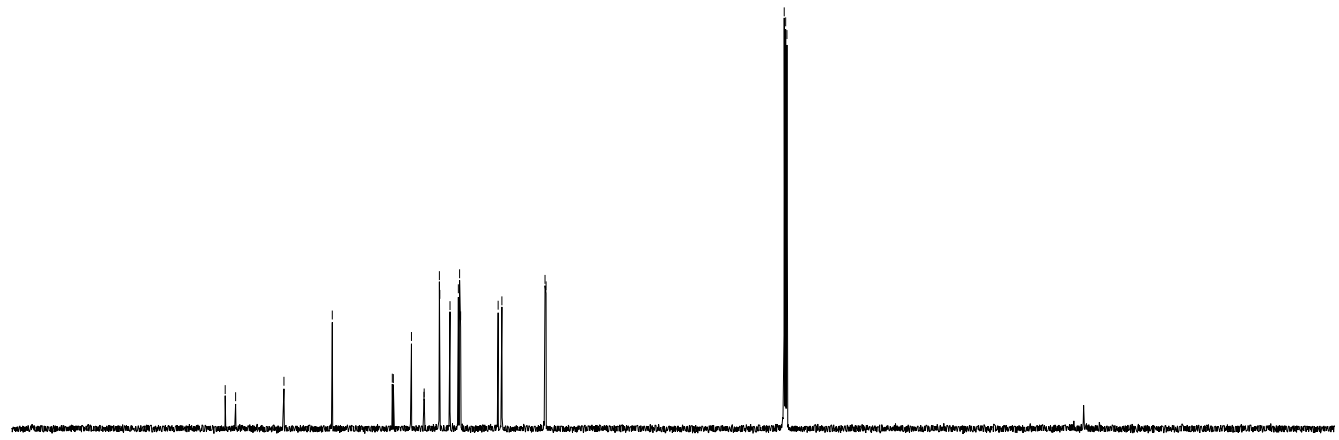

$\begin{array}{lllllllllll}200 & 190 & 180 & 170 & 160 & 150 & 140 & 130 & 120 & 110 & 100 \\ \mathrm{fl}(\mathrm{ppm}) & 90\end{array}$ 


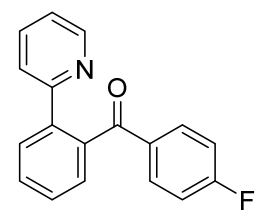

$12,565 \mathrm{MHz} \mathrm{CDCl}_{3}$

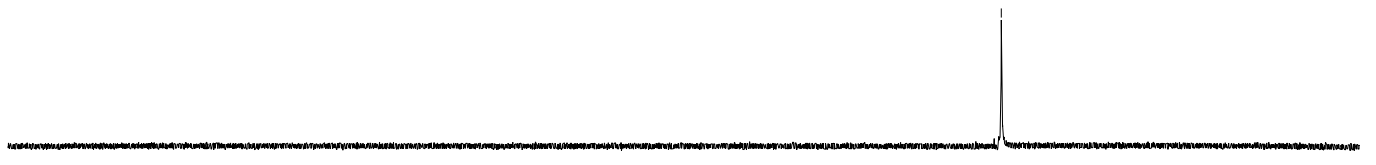

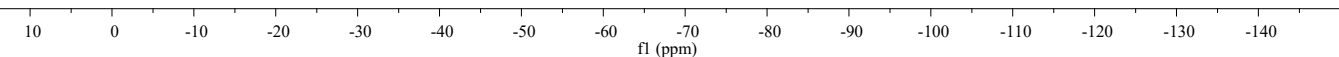


Yن

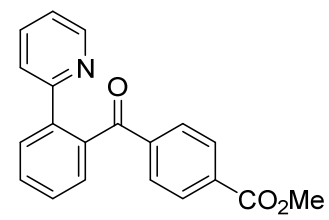

$13,600 \mathrm{MHz}, \mathrm{CDCl}_{3}$
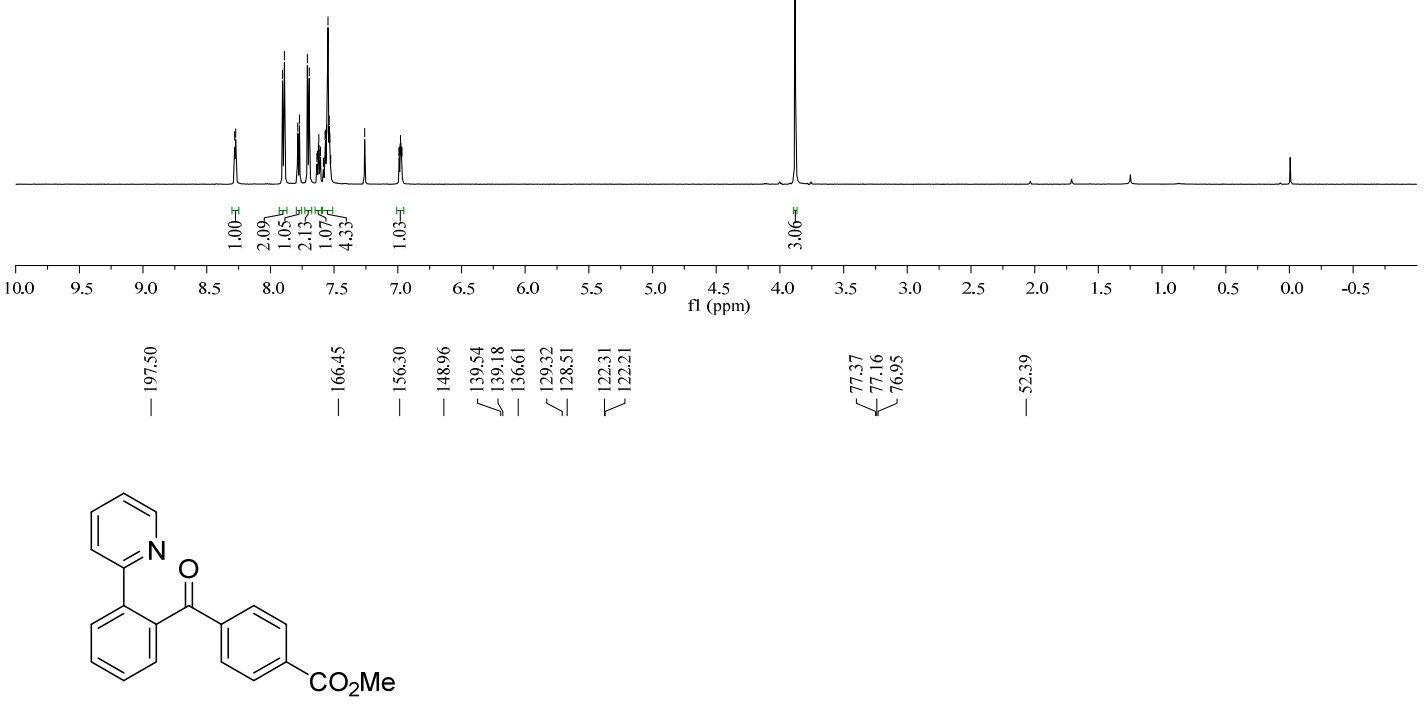

13, $150 \mathrm{MHz}, \mathrm{CDCl}_{3}$

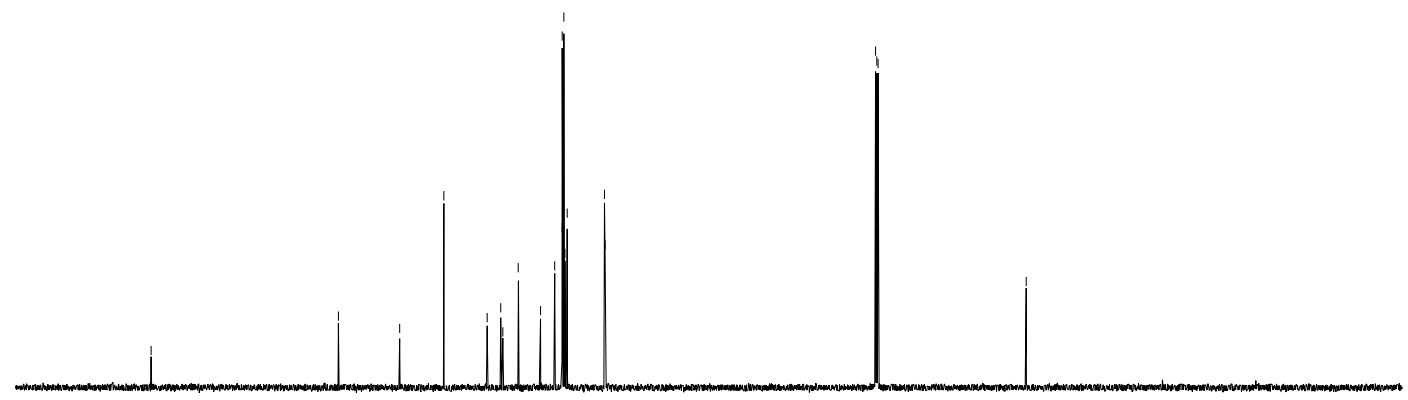

$\begin{array}{rlllllllllllllllllllllllllllll}1 & 220 & 210 & 200 & 190 & 180 & 170 & 160 & 150 & 140 & 130 & 120 & \begin{array}{c}110 \\ \mathrm{fl}(\mathrm{ppm})\end{array} & 100 & 90 & 70 & 60 & 50 & 40 & 30 & 20 & 10 & 0 & -10\end{array}$ 


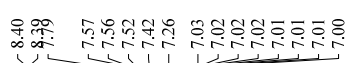

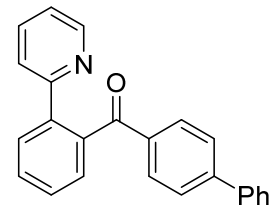

$14,600 \mathrm{MHz}, \mathrm{CDCl}_{3}$
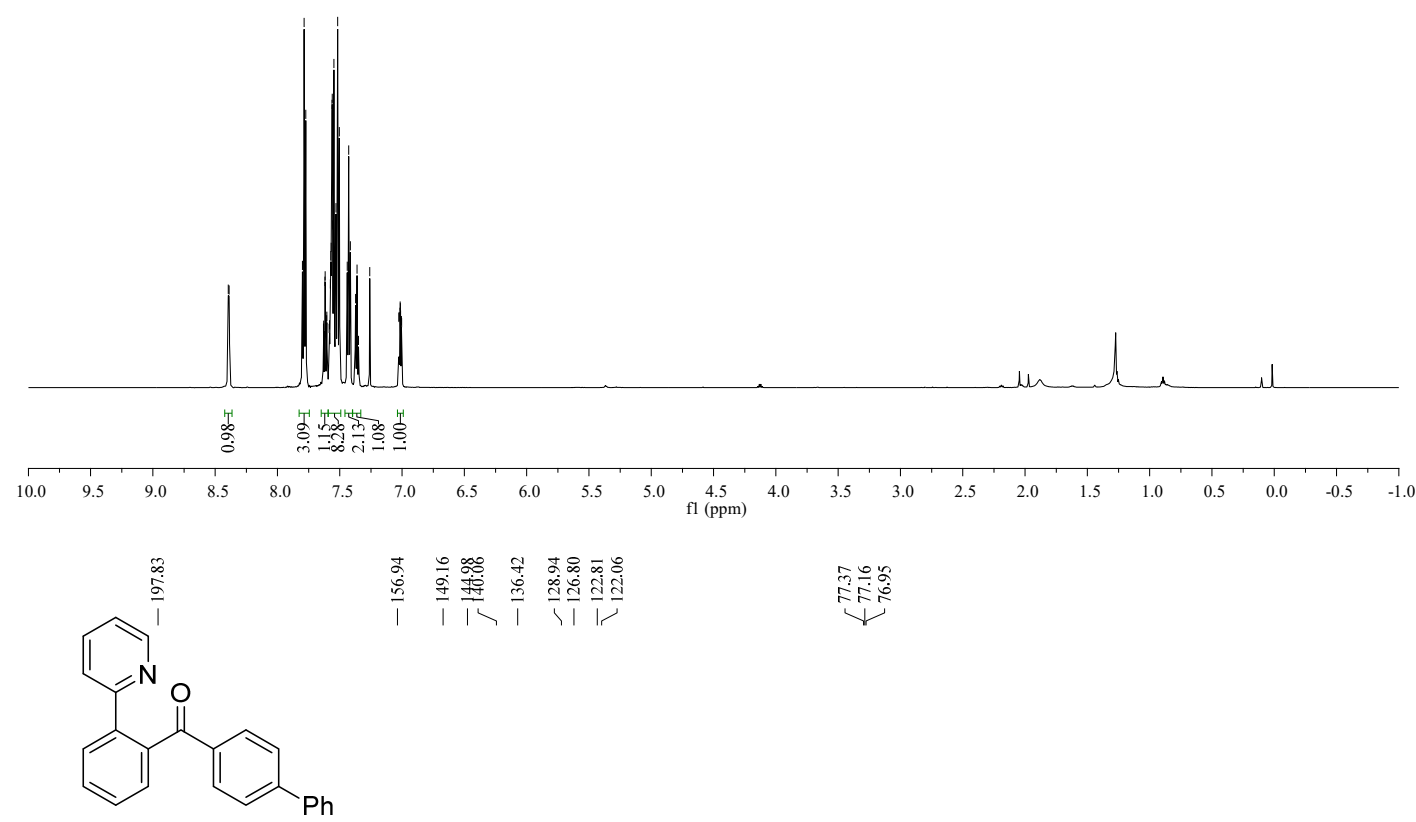

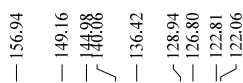

$\sqrt{\sqrt[3]{3} \frac{0}{2}}$

14, $150 \mathrm{MHz}, \mathrm{CDCl}_{3}$

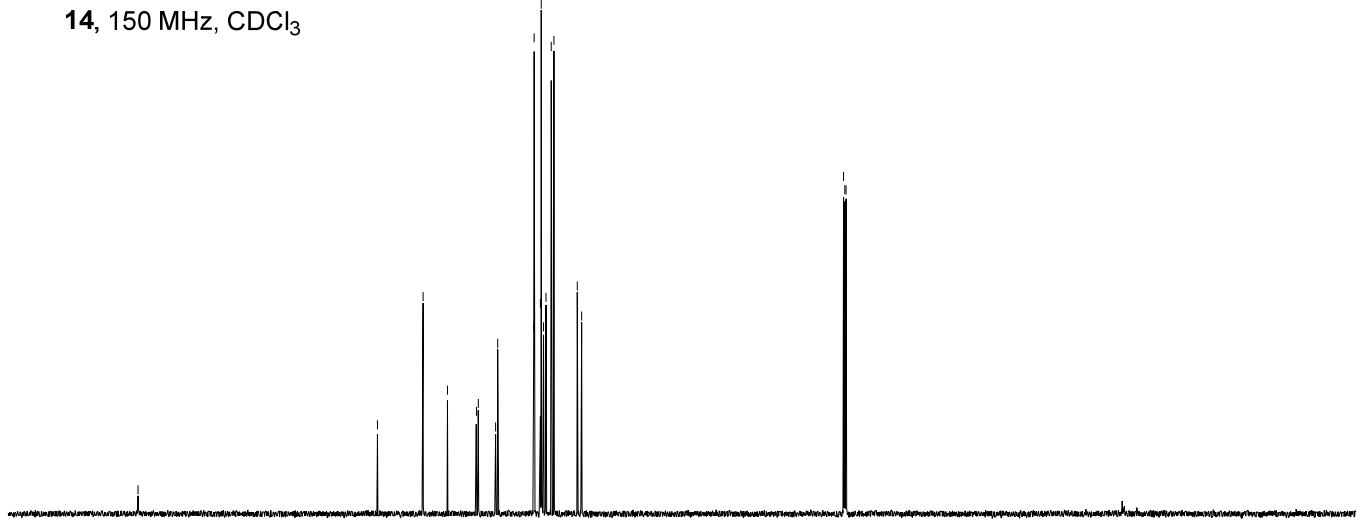

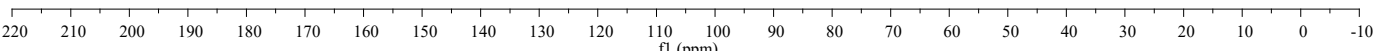




\section{$\underbrace{\sqrt{2}}$}

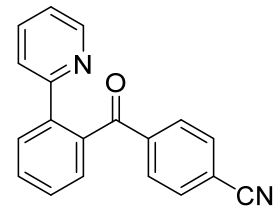

$15,600 \mathrm{MHz}, \mathrm{CDCl}_{3}$
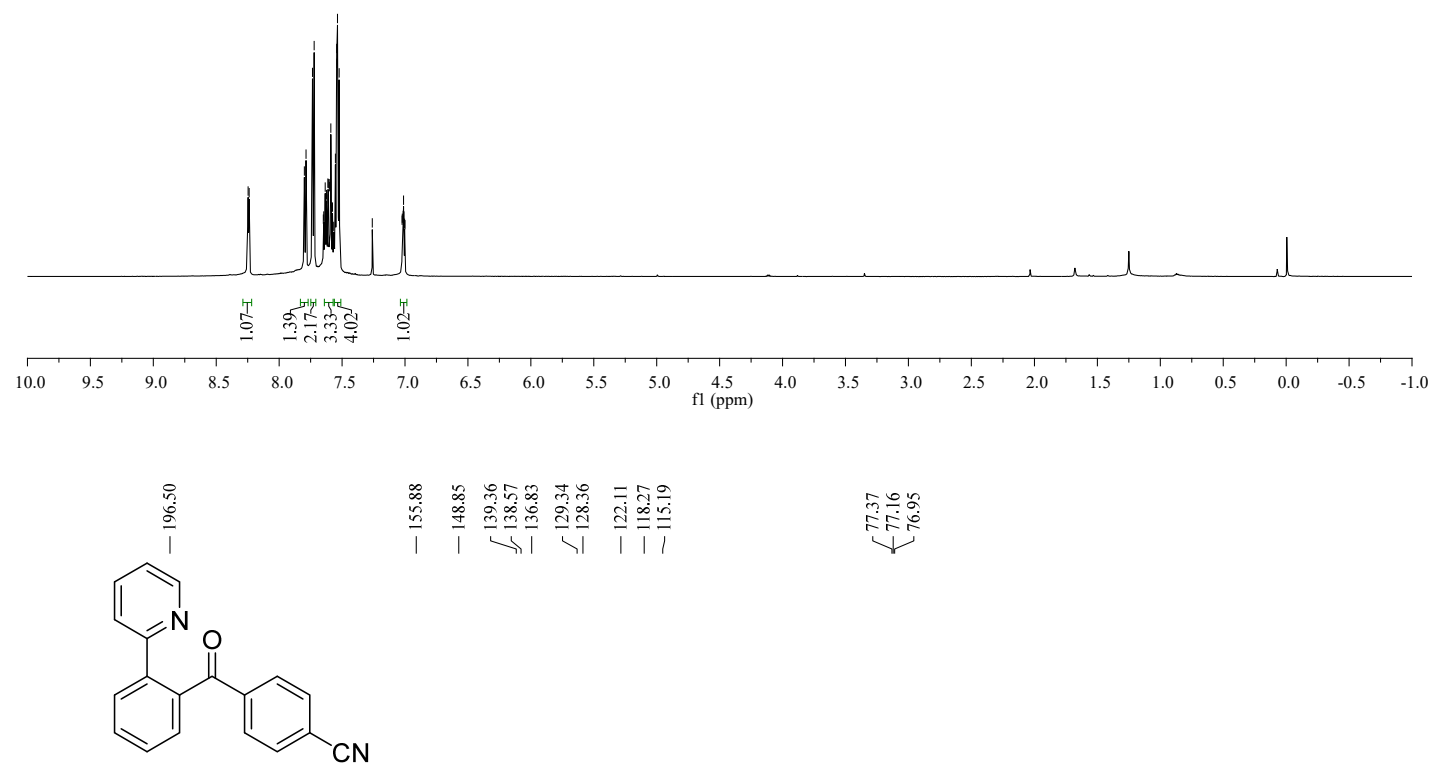

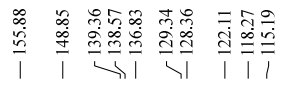

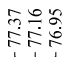

$15,150 \mathrm{MHz}, \mathrm{CDCl}_{3}$

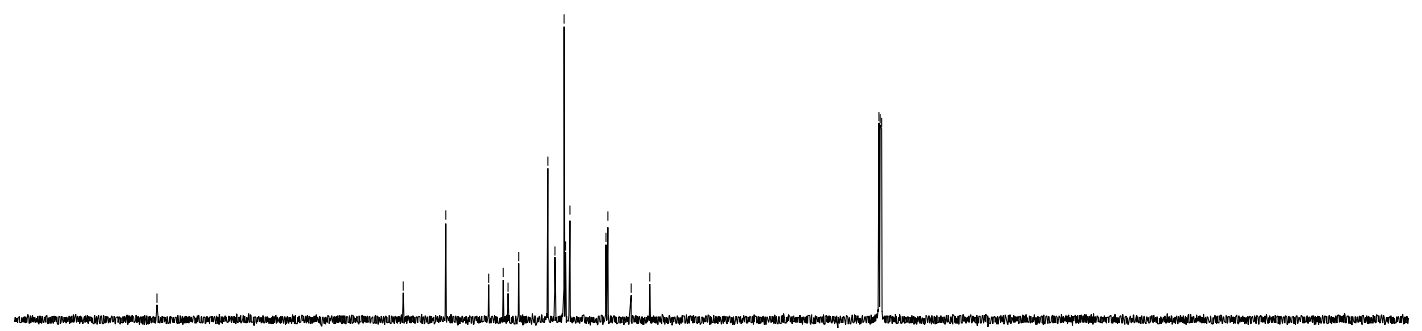

$\begin{array}{llllllllllllllllllllllll}220 & 210 & 200 & 190 & 180 & 170 & 160 & 150 & 140 & 130 & 120 & 110 & 100 & 90 & 80 & 70 & 60 & 50 & 40 & 30 & 20 & 10 & 0 & -10\end{array}$ 


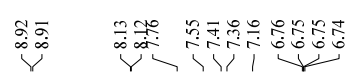

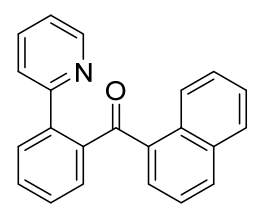

16, $600 \mathrm{MHz}, \mathrm{CDCl}_{3}$

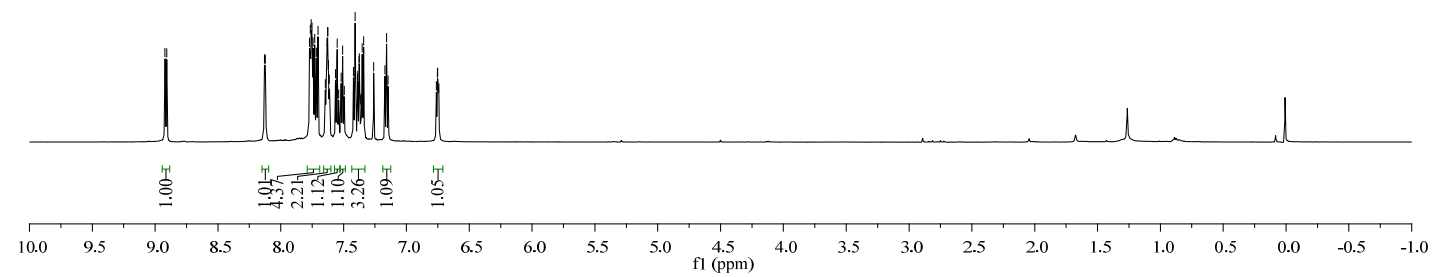

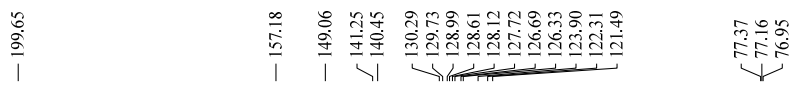

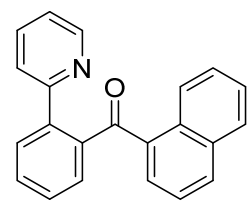

16, $150 \mathrm{MHz}, \mathrm{CDCl}_{3}$

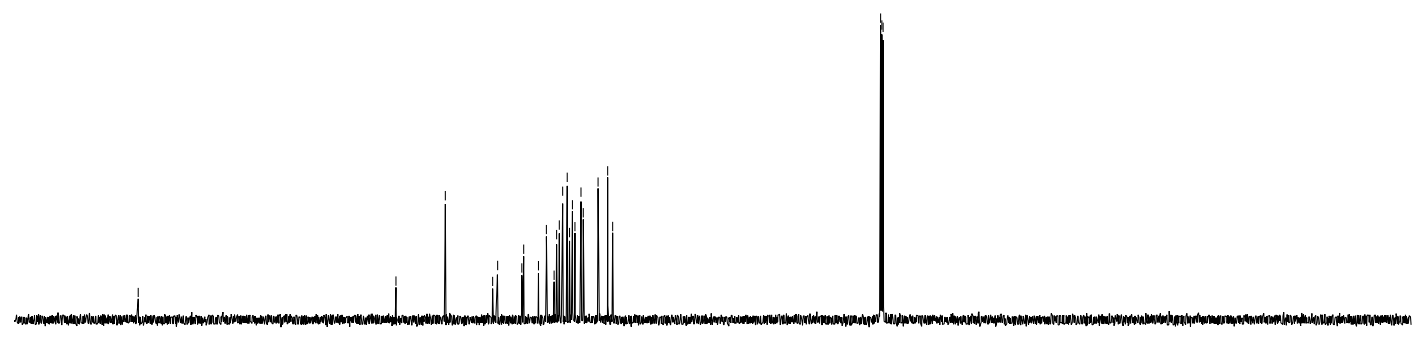

$\begin{array}{lllllllllllllllllllllllllllllllllll}220 & 210 & 200 & 190 & 180 & 170 & 160 & 150 & 140 & 130 & 120 & 110 & 100 & 90 & 80 & 70 & 60 & 50 & 40 & 30 & 20 & 10 & 0 & -10\end{array}$ 


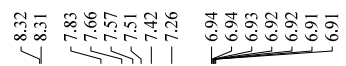

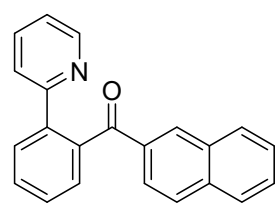

17, $400 \mathrm{MHz}, \mathrm{CDCl}_{3}$

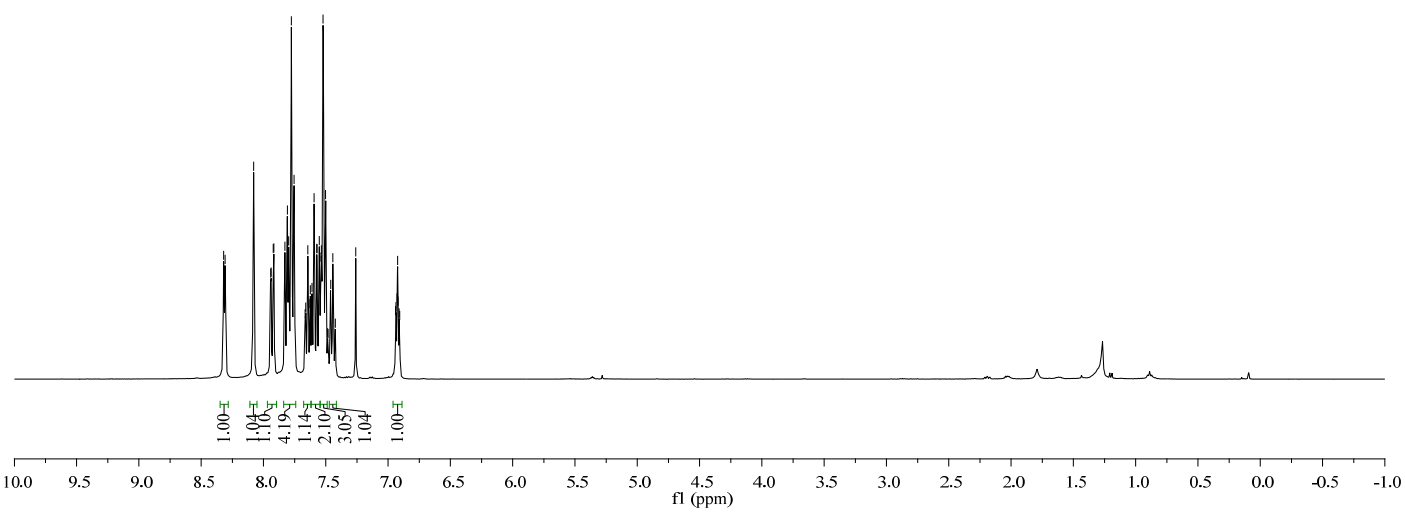

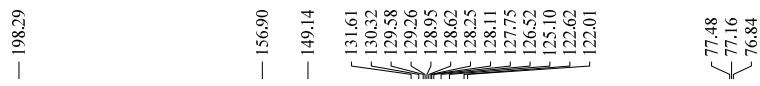

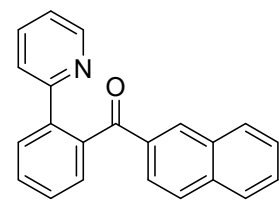

17, $100 \mathrm{MHz}, \mathrm{CDCl}_{3}$

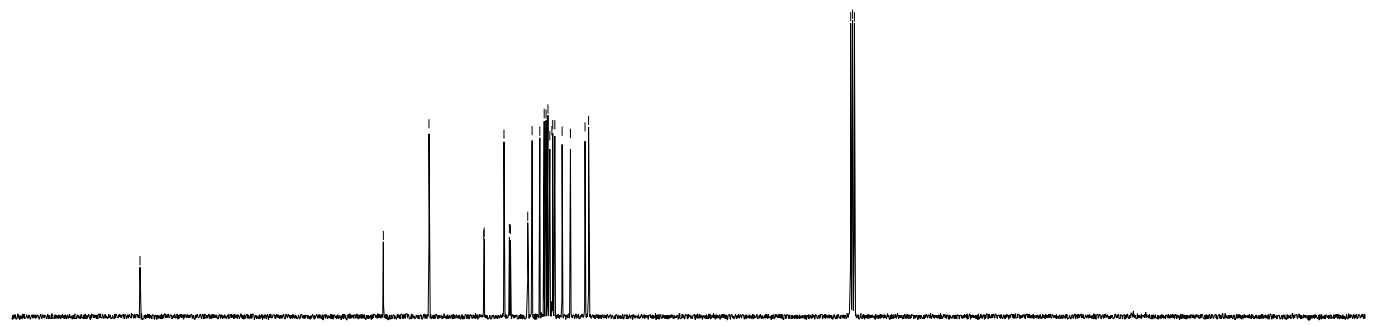

$\begin{array}{lllllllllllllllllllllllll}1 & 220 & 210 & 200 & 190 & 180 & 170 & 160 & 150 & 140 & 130 & 120 & \underset{\mathrm{fl}}{1}(\mathrm{ppm}) & 100 & 90 & 80 & 70 & 60 & 50 & 40 & 30 & 20 & 10 & 0 & -10\end{array}$ 


\section{Y)}

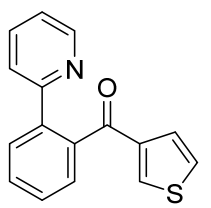

$18,600 \mathrm{MHz}, \mathrm{CDCl}_{3}$
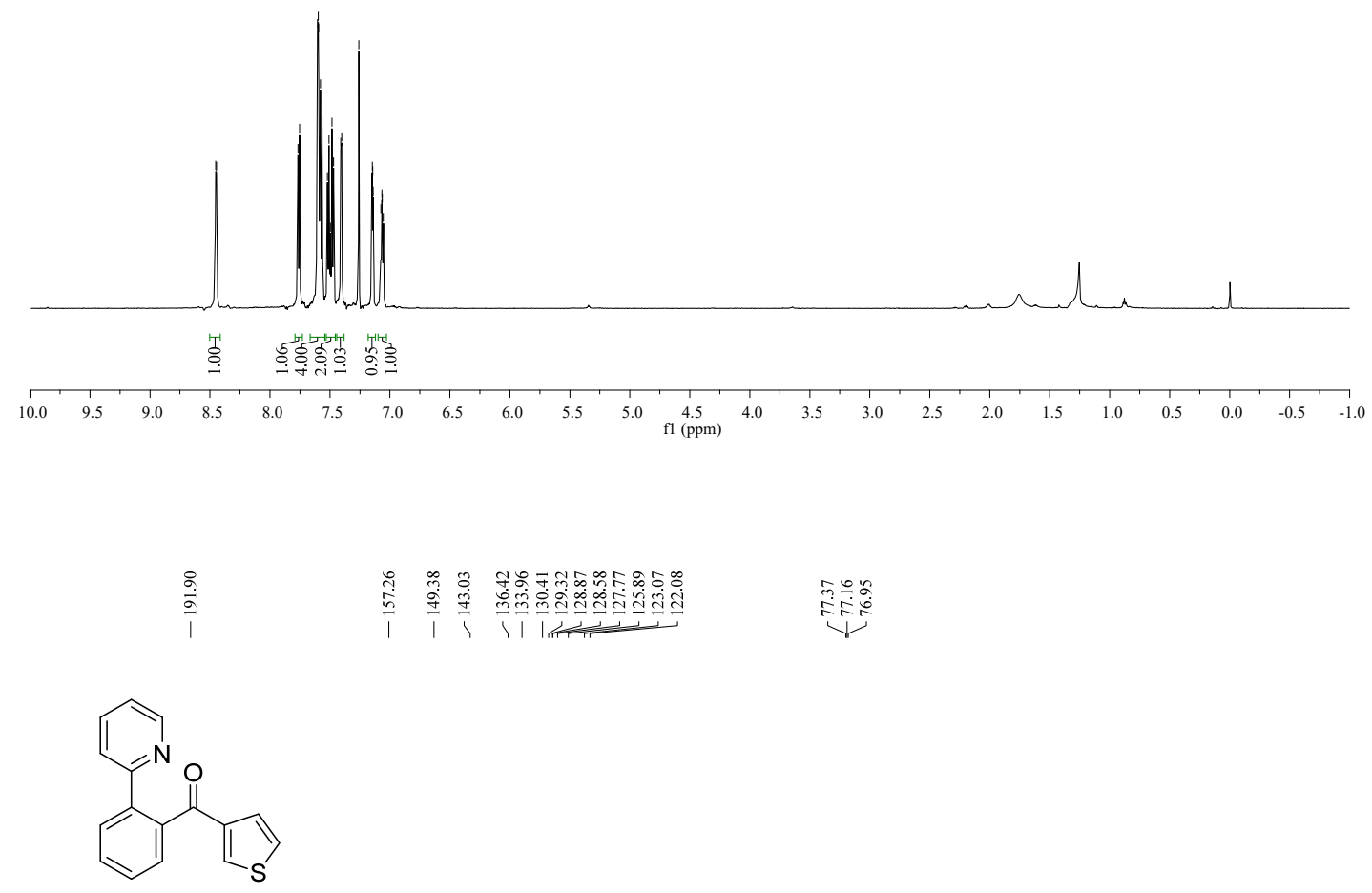

18, $150 \mathrm{MHz}, \mathrm{CDCl}_{3}$

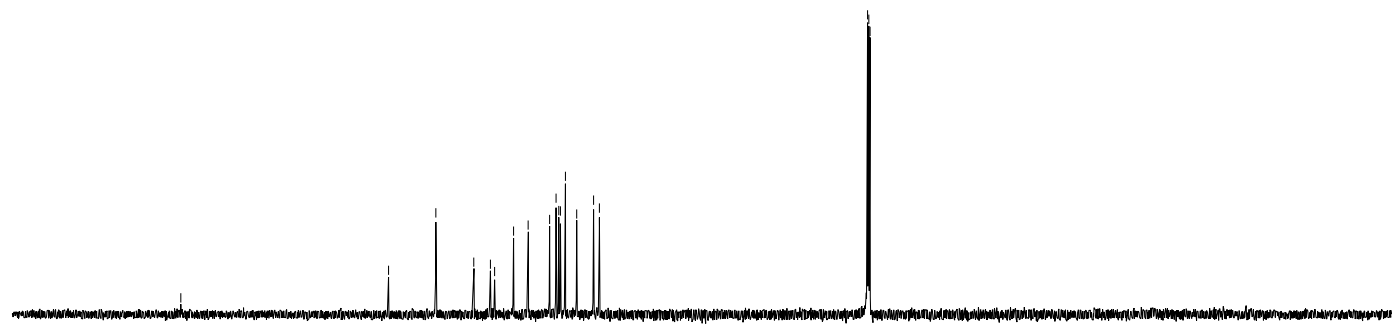

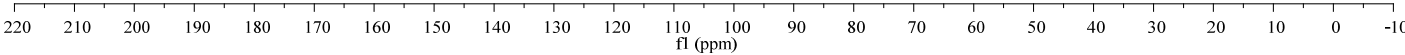




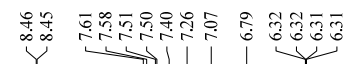

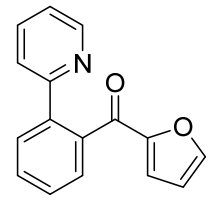

19, $600 \mathrm{MHz}, \mathrm{CDCl}_{3}$

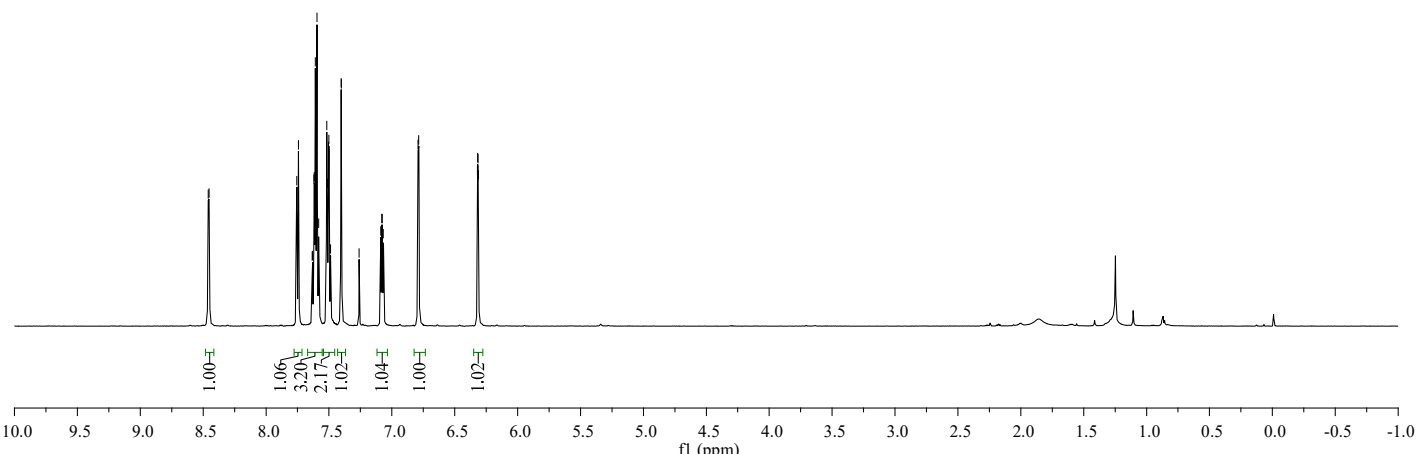

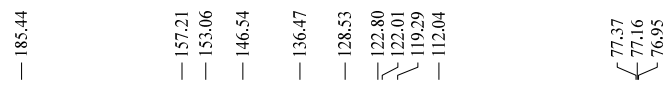

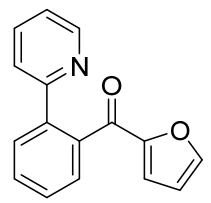

$19,150 \mathrm{MHz}, \mathrm{CDCl}_{3}$

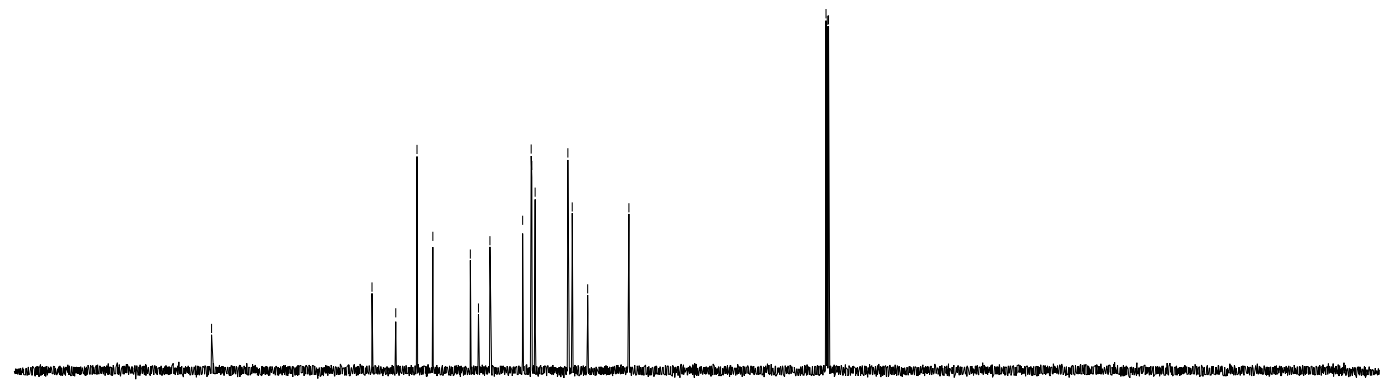

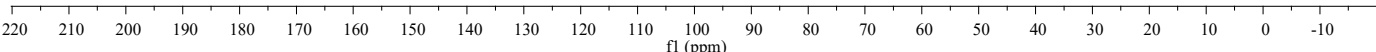


$\underbrace{\underbrace{\prime}}$

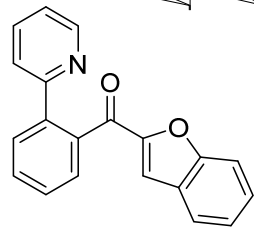

20, $600 \mathrm{MHz}, \mathrm{CDCl}_{3}$

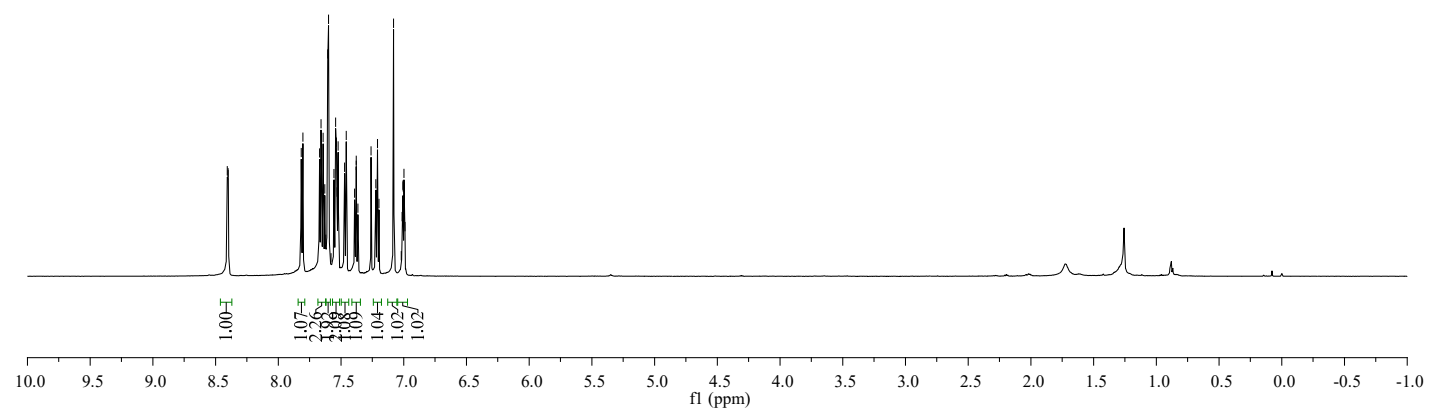

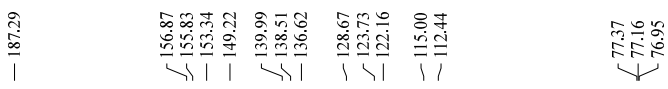

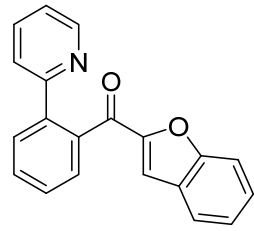

20, $150 \mathrm{MHz}, \mathrm{CDCl}_{3}$

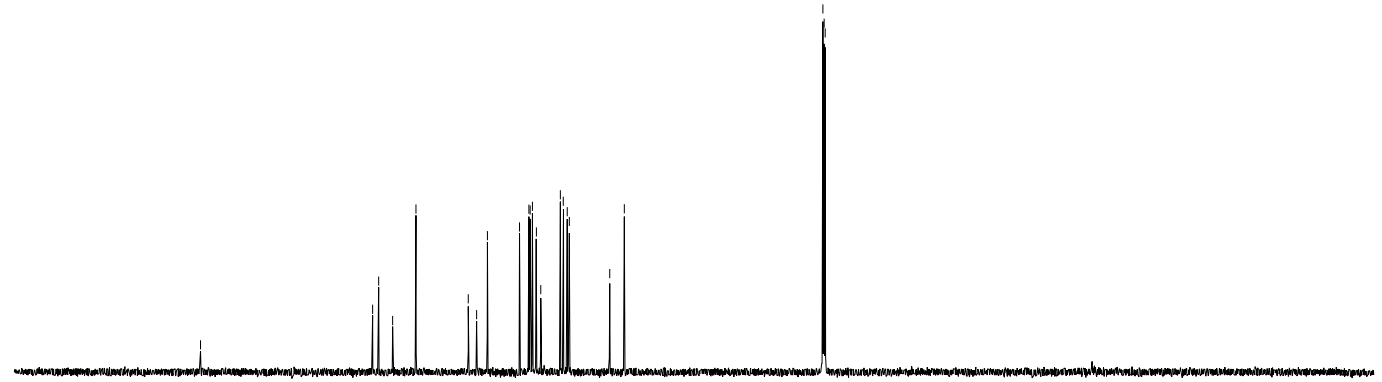

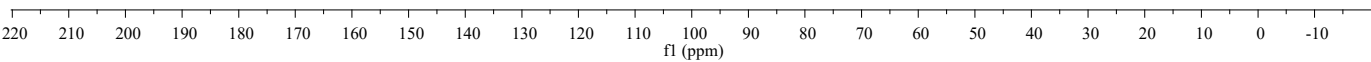




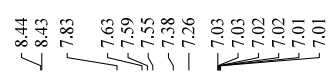

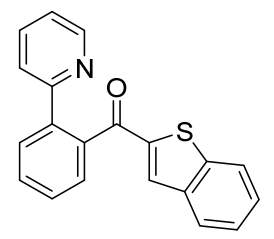

21, $600 \mathrm{MHz}, \mathrm{CDCl}_{3}$

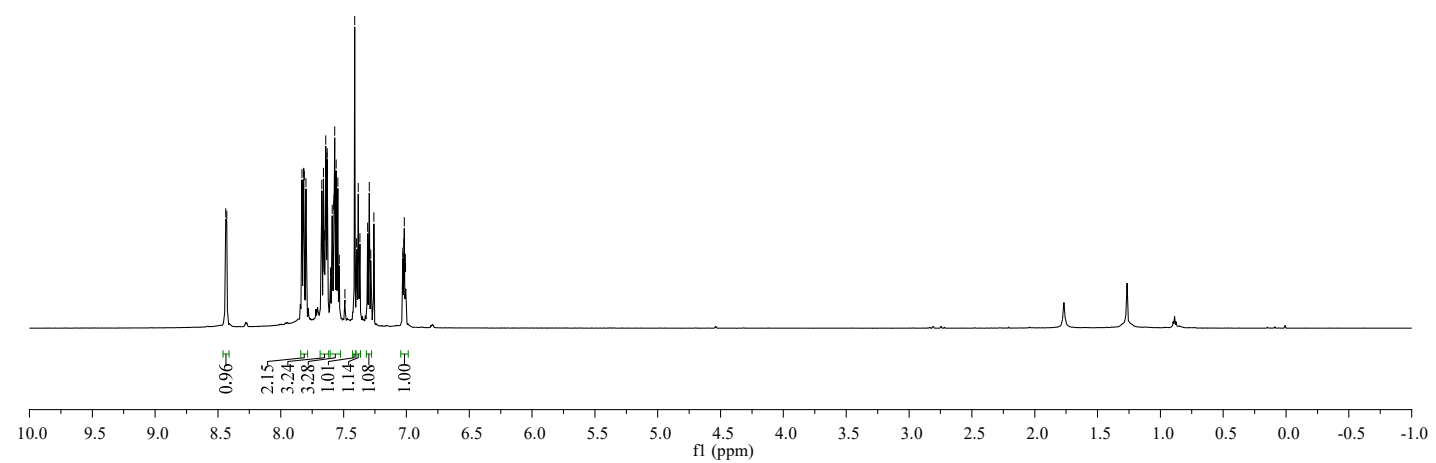

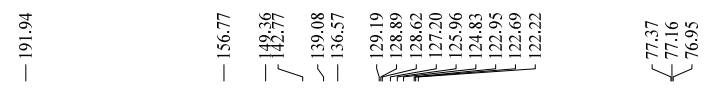

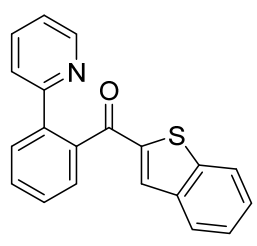

21, $150 \mathrm{MHz}, \mathrm{CDCl}_{3}$

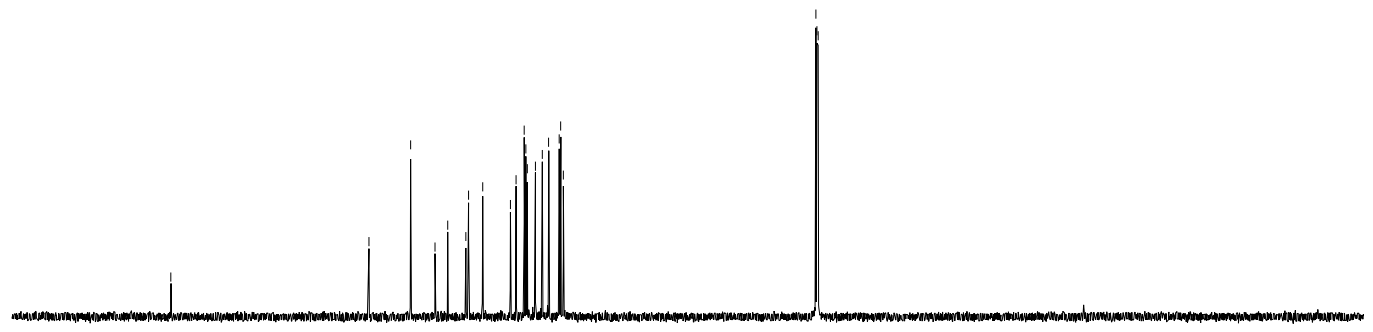

$\begin{array}{llllllllllllllllllllllllllllllllll} & 220 & 210 & 200 & 190 & 180 & 170 & 160 & 150 & 140 & 130 & 120 & 110 & 100 & 90 & 80 & 70 & 60 & 50 & 40 & 30 & 20 & 10 & 0 & -10\end{array}$ 
染

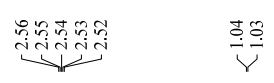

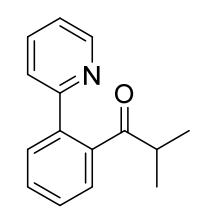

22, $600 \mathrm{MHz}, \mathrm{CDCl}_{3}$

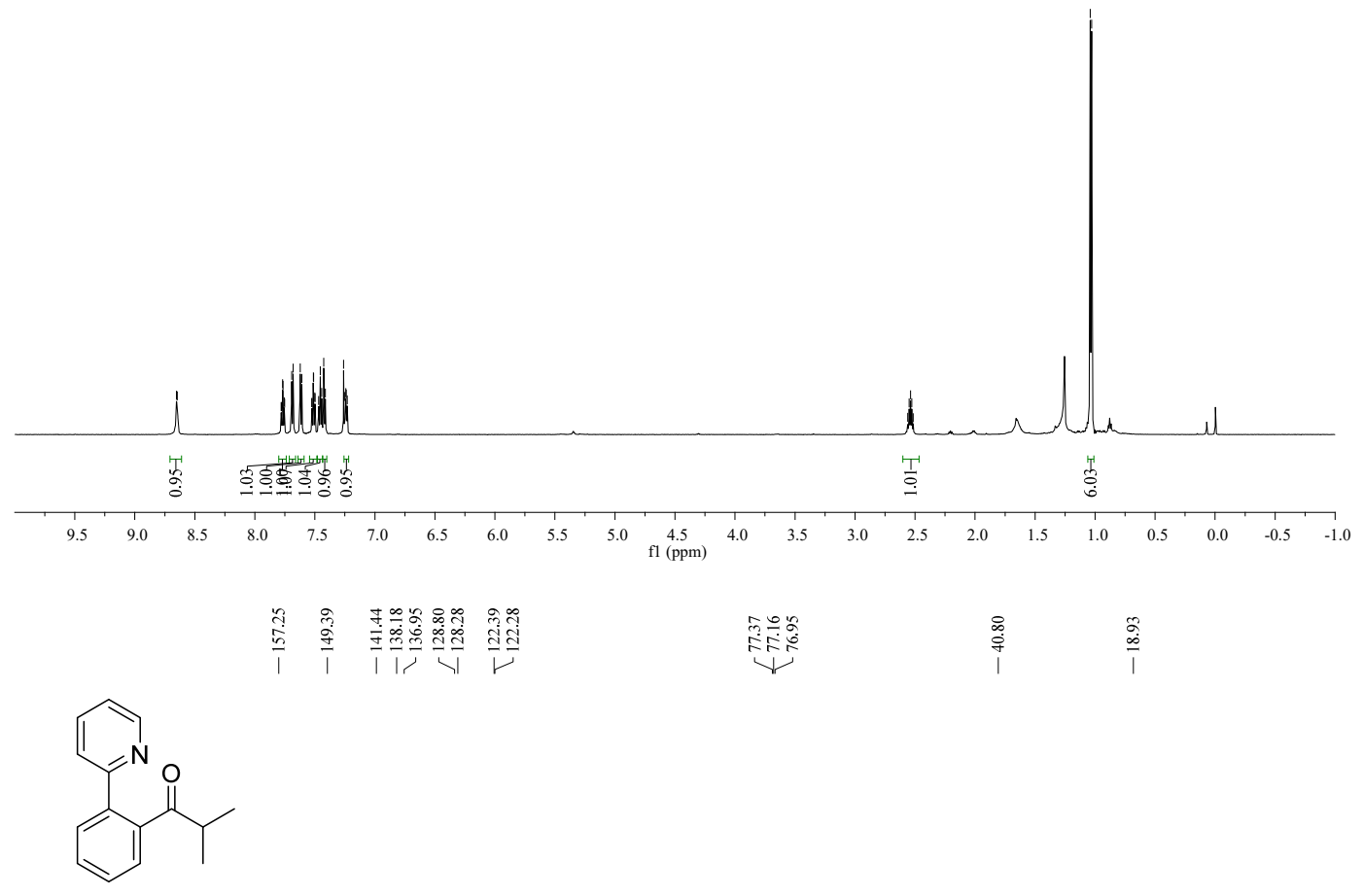

22, $150 \mathrm{MHz}, \mathrm{CDCl}_{3}$

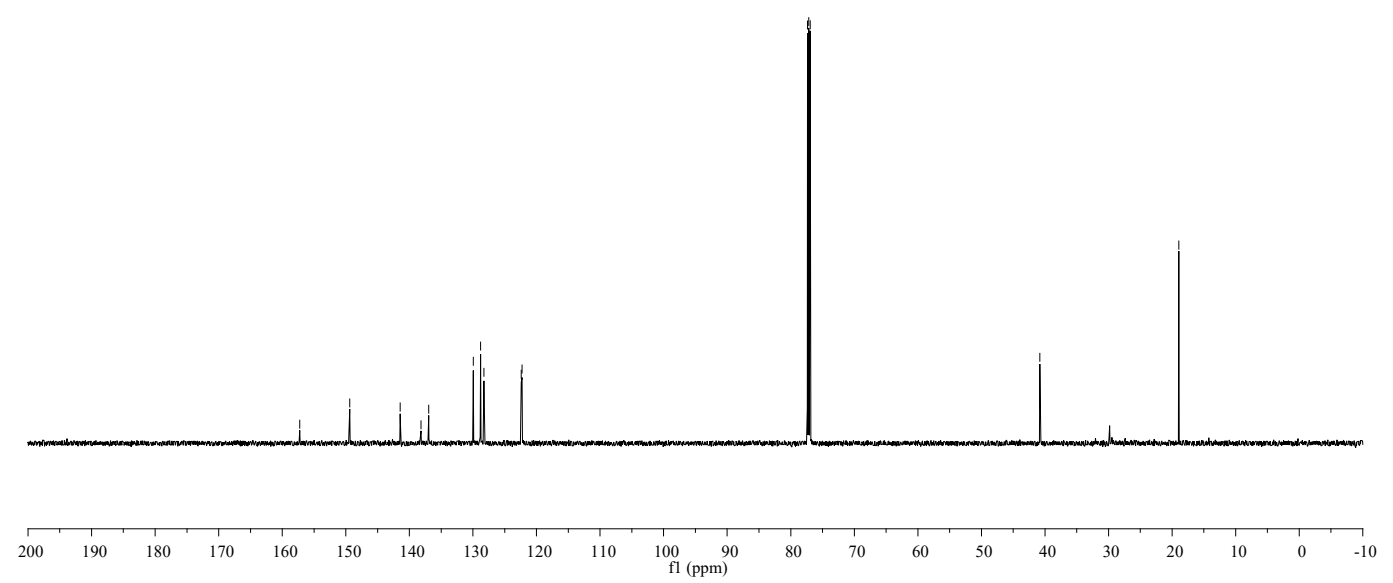




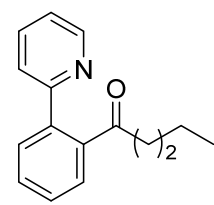

23, $600 \mathrm{MHz}, \mathrm{CDCl}_{3}$

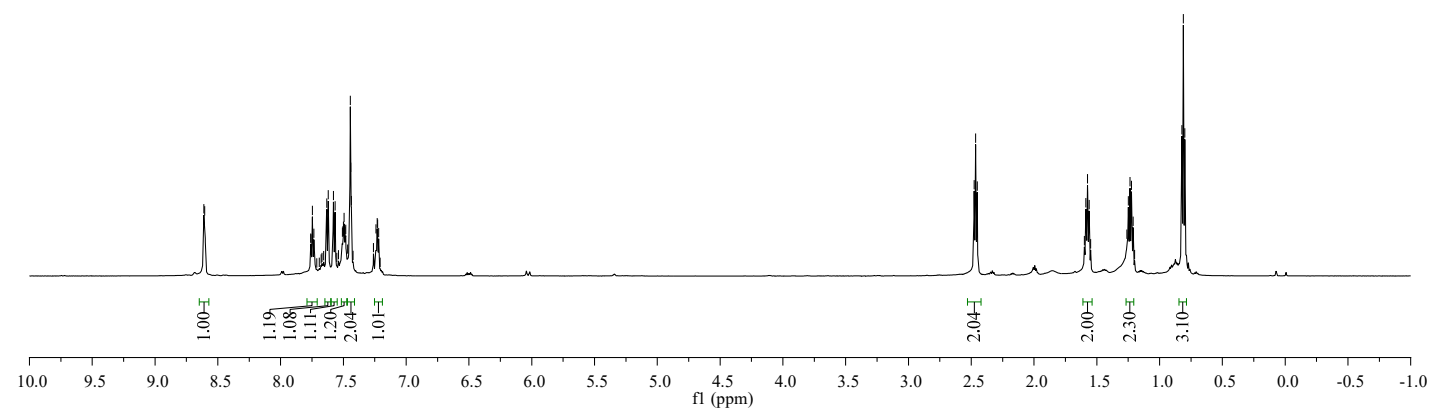

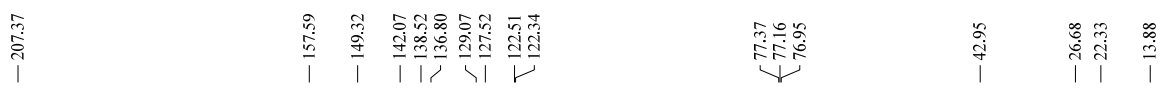

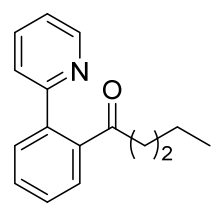

23, $150 \mathrm{MHz}, \mathrm{CDCl}_{3}$

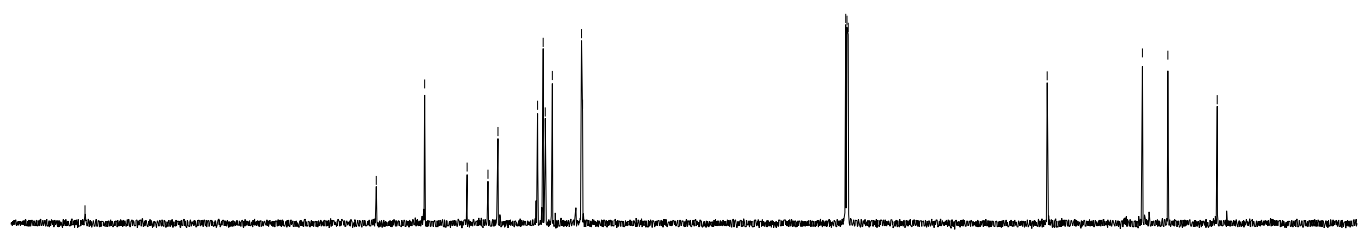

$\begin{array}{rrrrrrrrrrrrrrrrrrrrrrrr}1 & 1 \\ 220 & 210 & 200 & 190 & 180 & 170 & 160 & 150 & 140 & 130 & 120 & 110 & 100 & 90 & 80 & 70 & 60 & 50 & 40 & 30 & 20 & 10 & 0 & -10\end{array}$ 


\section{品总}

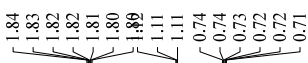

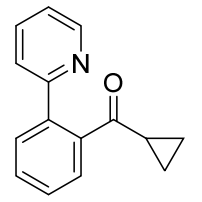

24, $600 \mathrm{MHz}, \mathrm{CDCl}_{3}$

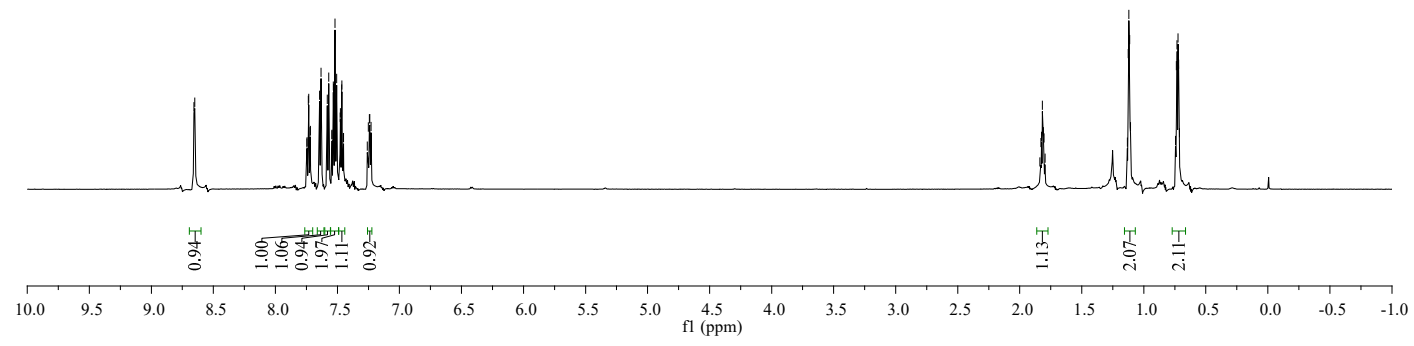

ปี

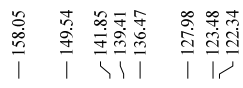

证

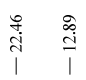

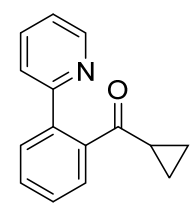

24, $150 \mathrm{MHz}, \mathrm{CDCl}_{3}$

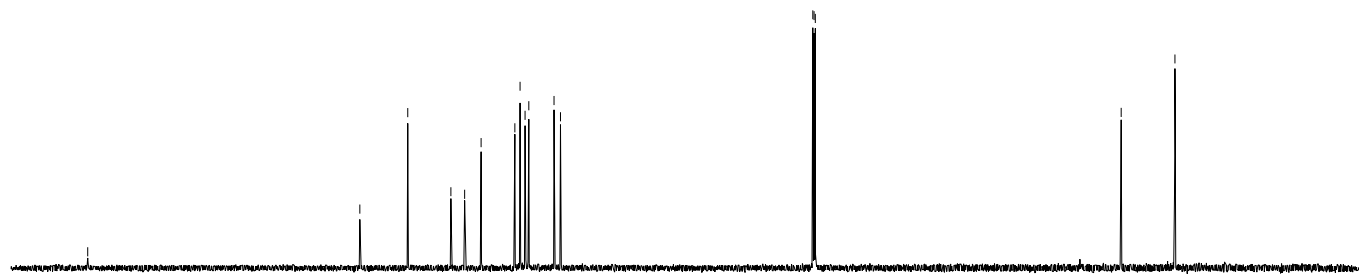

$\begin{array}{rllllllllllllllllllllllll}1 & 220 & 210 & 200 & 190 & 180 & 170 & 160 & 150 & 140 & 130 & 120 & 110 & 100 & 90 & 80 & 70 & 60 & 50 & 40 & 30 & 20 & 10 & 0 & -10\end{array}$ 


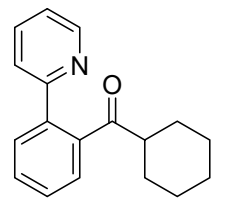

25, $600 \mathrm{MHz}, \mathrm{CDCl}_{3}$

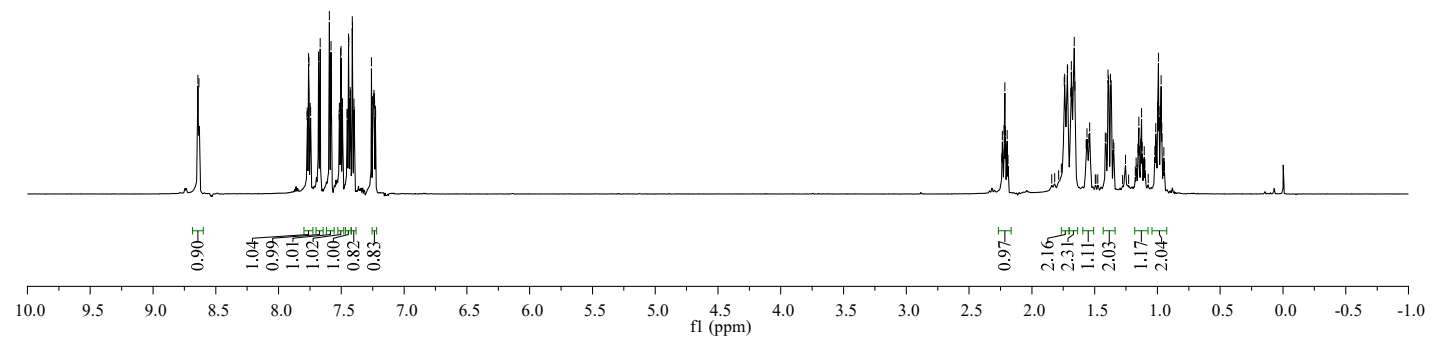

$\stackrel{\infty}{\stackrel{\infty}{N}}$

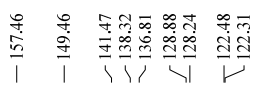

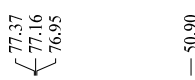

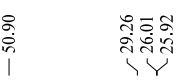

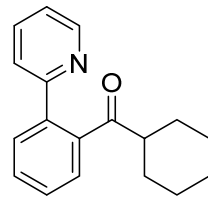

25, $150 \mathrm{MHz}, \mathrm{CDCl}_{3}$

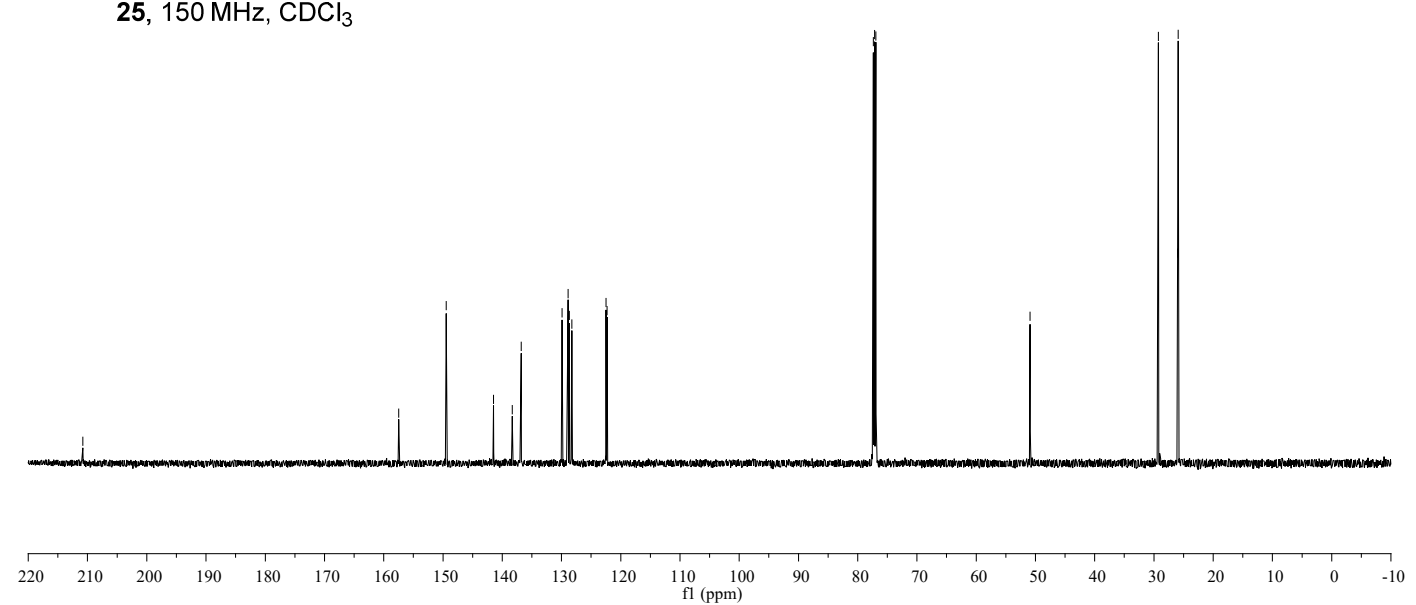




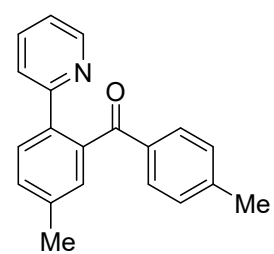

26, $600 \mathrm{MHz}, \mathrm{CDCl}_{3}$

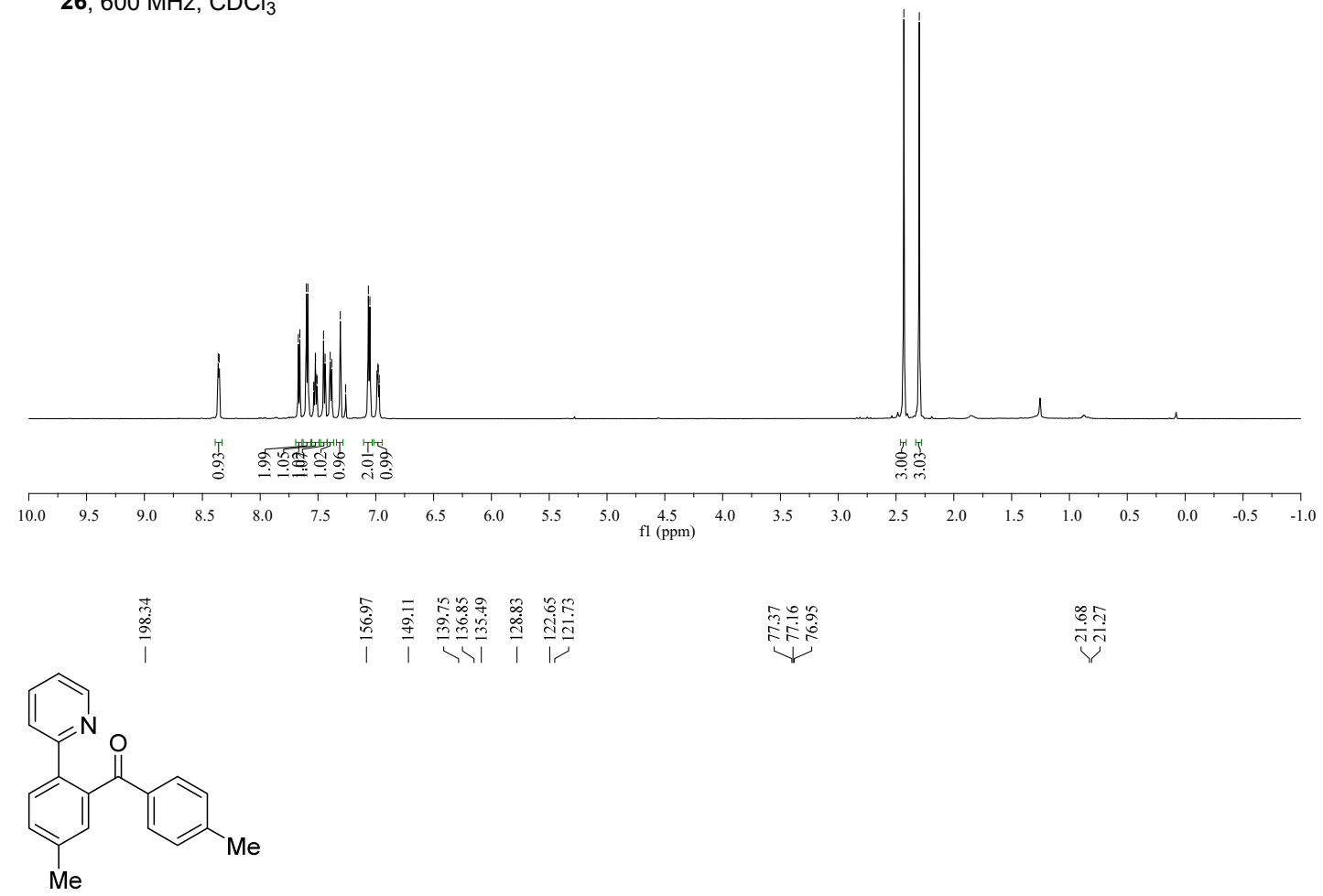

26, $150 \mathrm{MHz}, \mathrm{CDCl}_{3}$

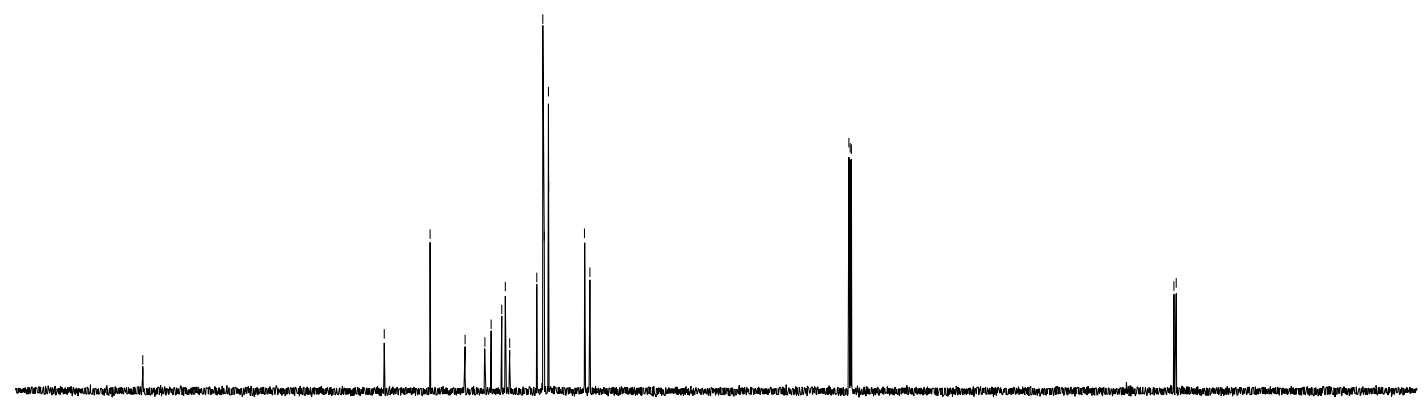

$\begin{array}{lllllllllllllllllllllllllll} & 220 & 210 & 200 & 190 & 180 & 170 & 160 & 150 & 140 & 130 & 120 & 110 & 100 & 90 & 80 & 70 & 60 & 50 & 40 & 30 & 20 & 10 & 0 & -10 & \end{array}$ 


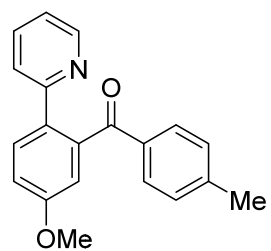

27, $600 \mathrm{MHz}, \mathrm{CDCl}_{3}$

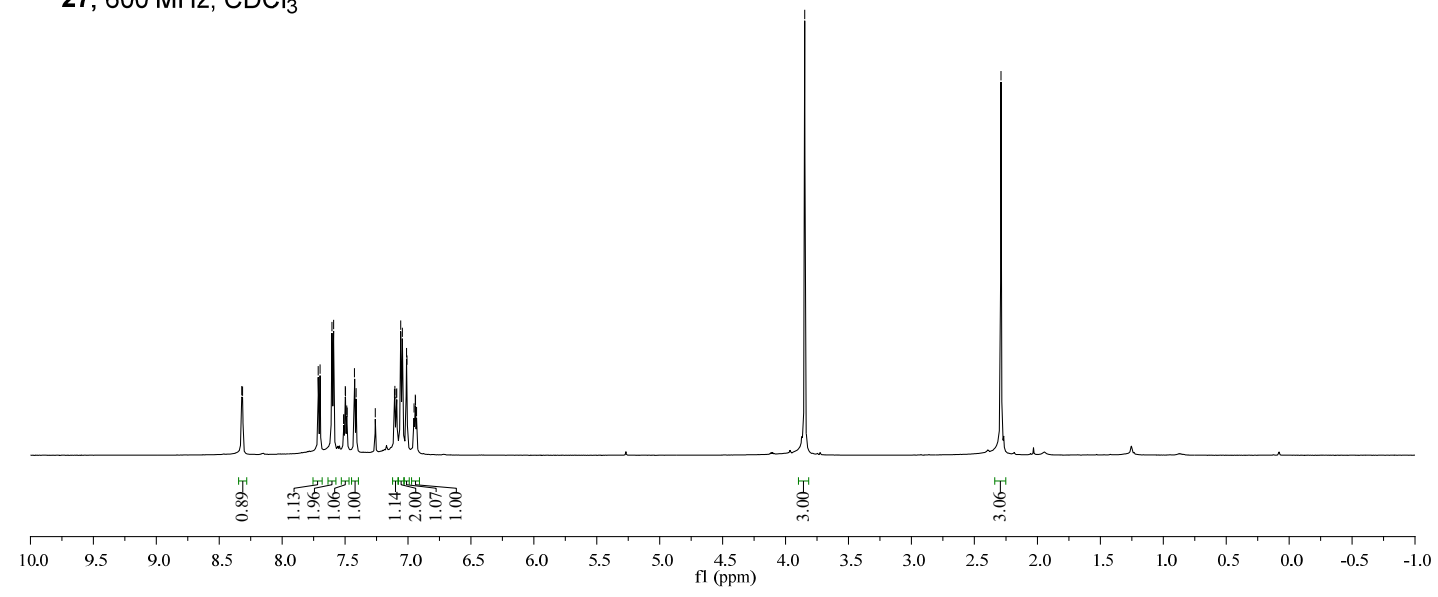

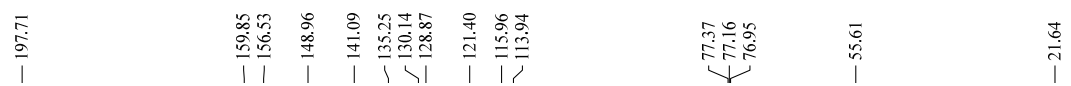

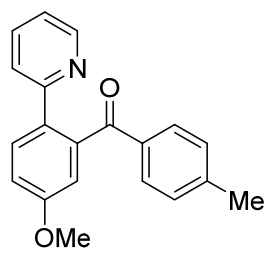

27, $150 \mathrm{MHz}, \mathrm{CDCl}_{3}$

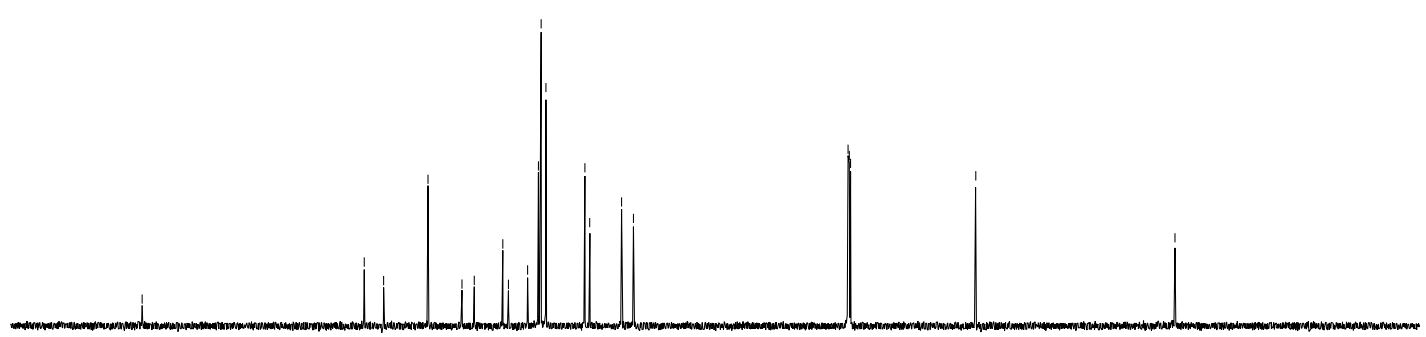

\begin{tabular}{rlllllllllllllllllllllllll}
\hline 220 & 210 & 200 & 190 & 180 & 170 & 160 & 150 & 140 & 130 & 120 & 110 & $\begin{array}{c}100 \\
\mathrm{fl}(\mathrm{ppm})\end{array}$ & 90 & 80 & 70 & 60 & 50 & 40 & 30 & 20 & 10 & 0 & -10
\end{tabular} 


\section{证年}

i

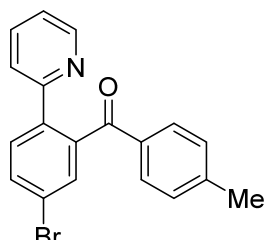

28, $600 \mathrm{MHz}, \mathrm{CDCl}_{3}$
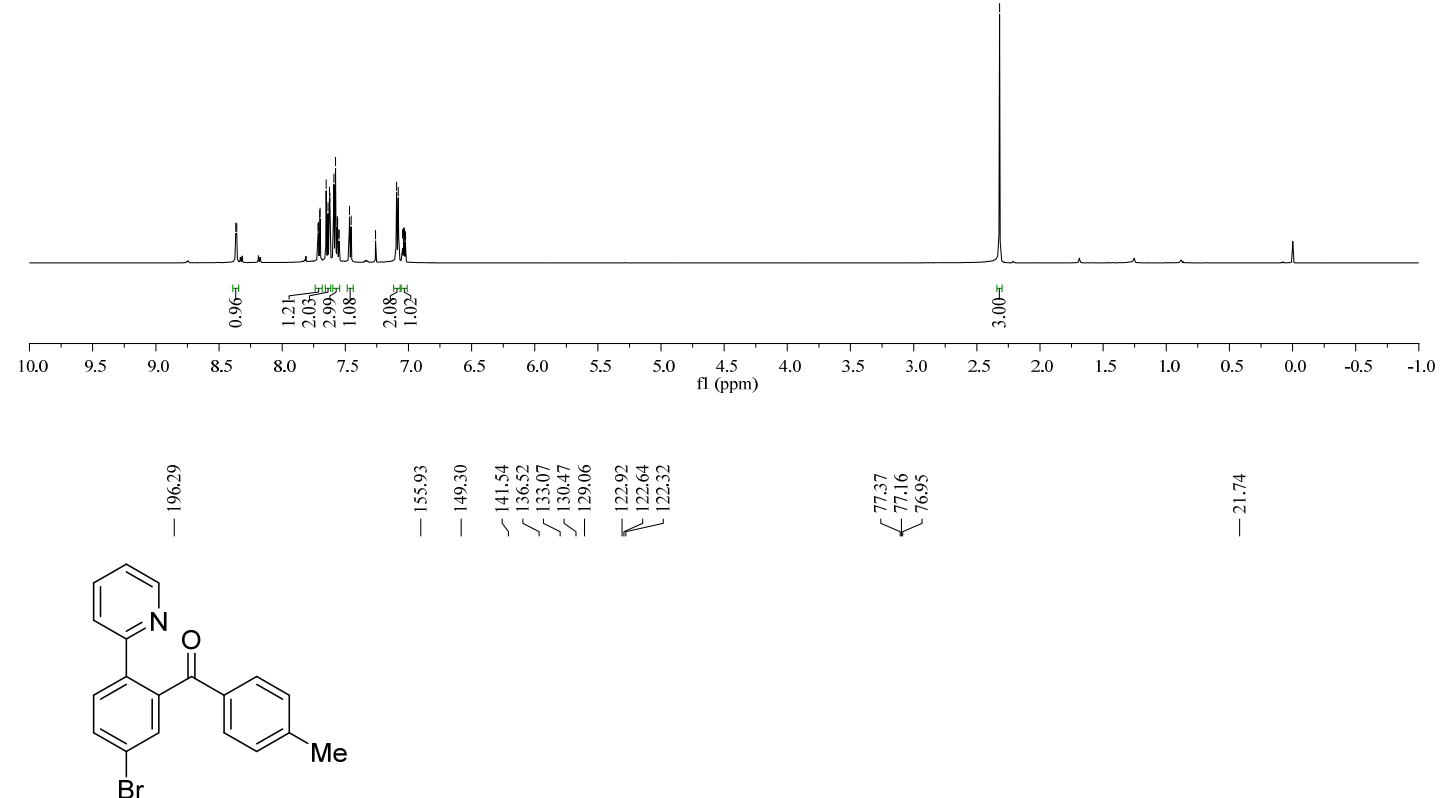

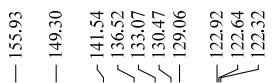

许

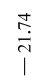

28, $150 \mathrm{MHz}, \mathrm{CDCl}_{3}$

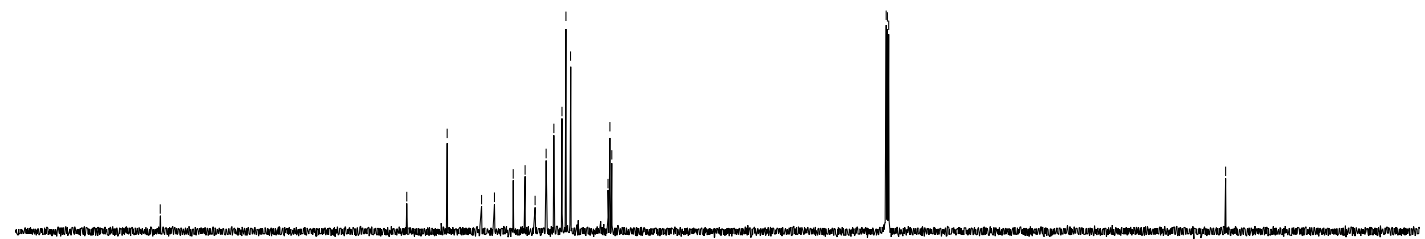

\begin{tabular}{rllllllllllllllllllllllllll}
\hline & 220 & 210 & 200 & 190 & 180 & 170 & 160 & 150 & 140 & 130 & 120 & 110 & 100 & 90 & 80 & 70 & 60 & 50 & 40 & 30 & 20 & 10 & 0 & -10
\end{tabular} 
Y

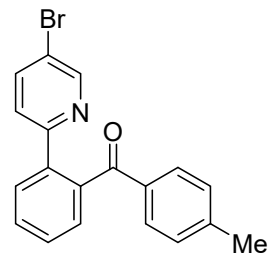

29, $600 \mathrm{MHz}, \mathrm{CDCl}_{3}$
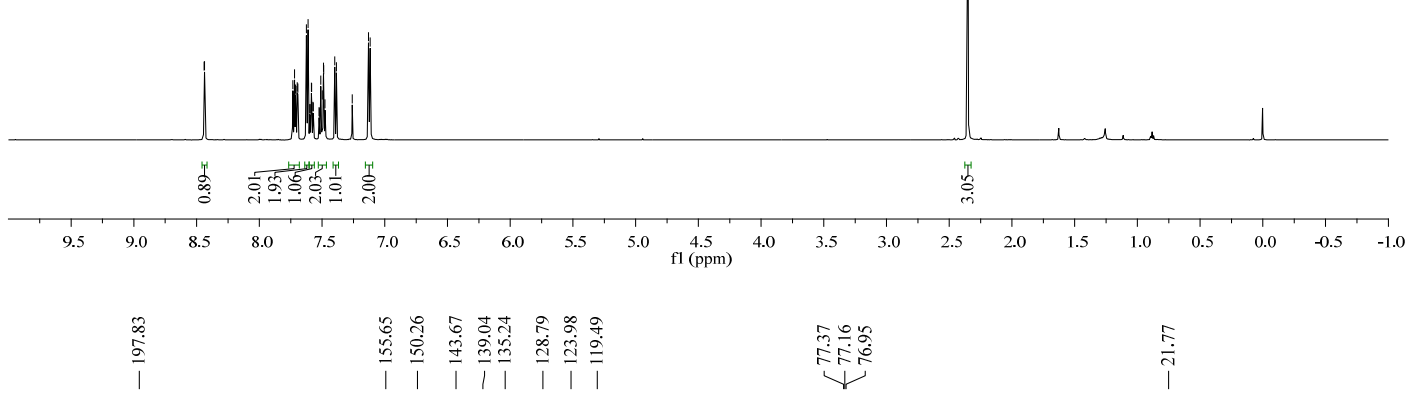

许

$\stackrel{\stackrel{N}{i}}{i}$

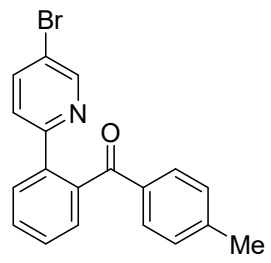

29, $150 \mathrm{MHz}, \mathrm{CDCl}_{3}$

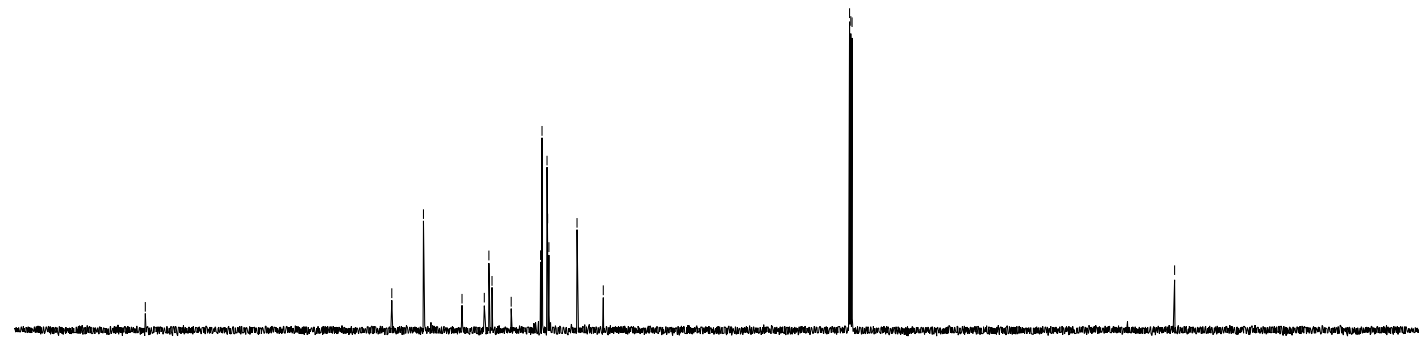

$\begin{array}{lllllllllllllllllllllllll}220 & 210 & 200 & 190 & 180 & 170 & 160 & 150 & 140 & 130 & 120 & 110 & 100 & 90 & 80 & 70 & 60 & 50 & 40 & 30 & 20 & 10 & 0 & -10\end{array}$ 


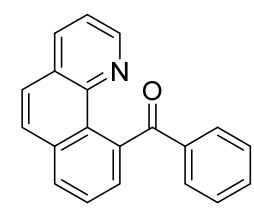

$30,600 \mathrm{MHz}, \mathrm{CDCl}_{3}$
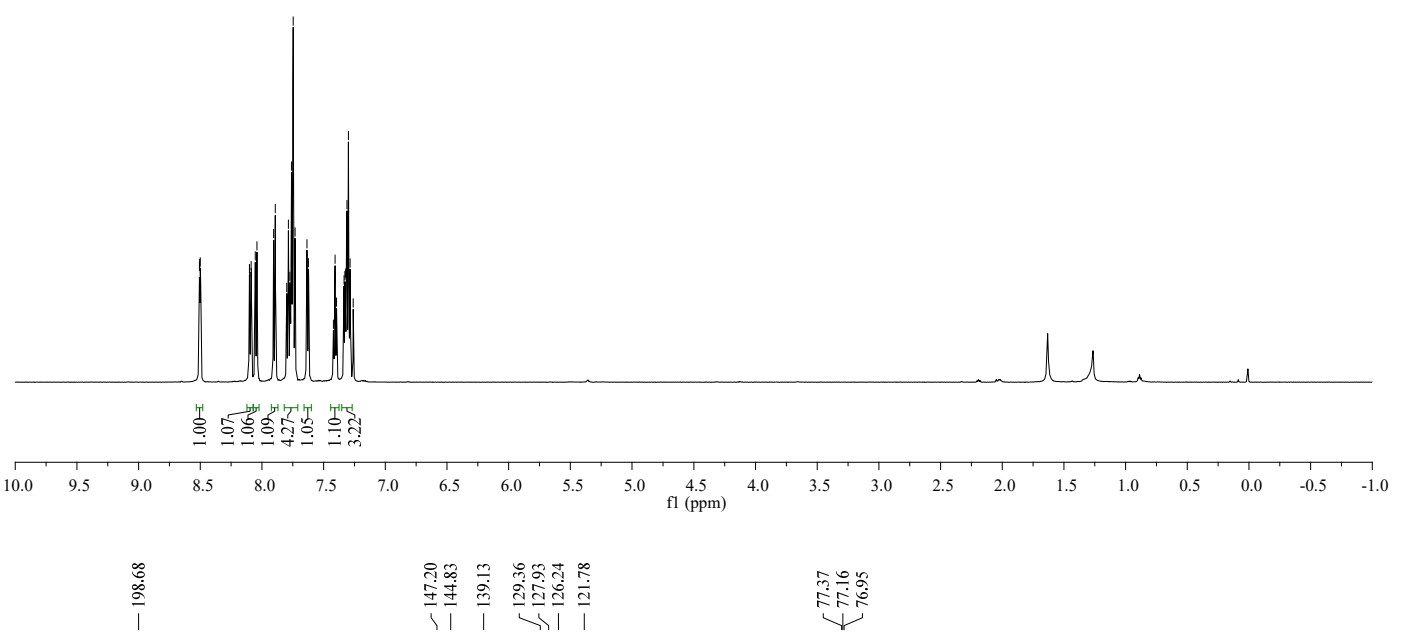

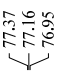

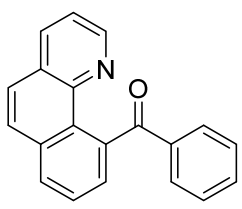

30, $150 \mathrm{MHz}, \mathrm{CDCl}_{3}$

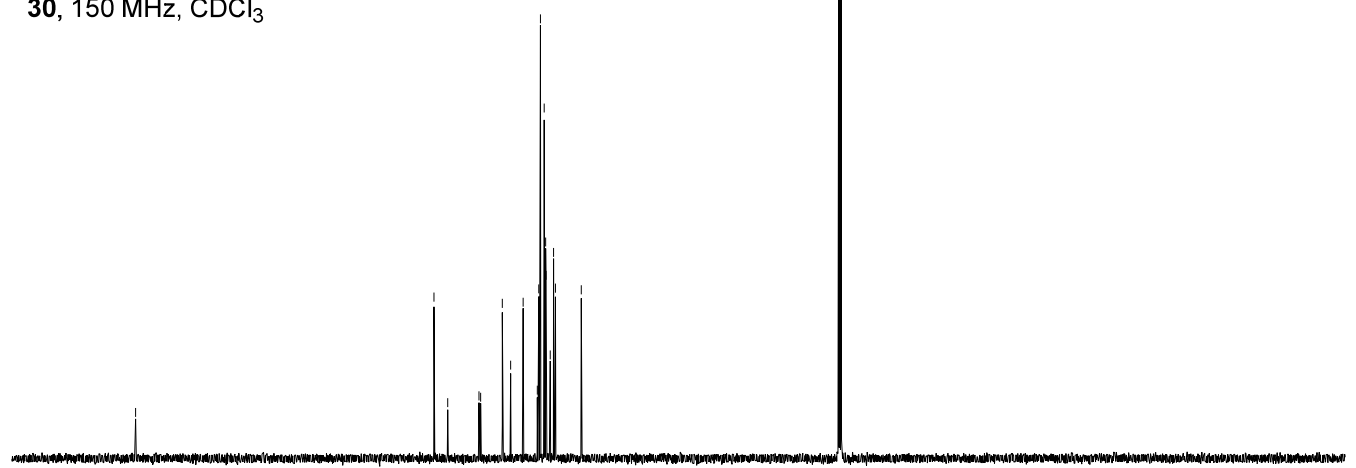

\begin{tabular}{rlllllllllllllllllllllllll}
\hline 220 & 210 & 200 & 190 & 180 & 170 & 160 & 150 & 140 & 130 & 120 & $\underset{f 1}{110}\left(\begin{array}{ll}100 \\
\mathrm{fpm})\end{array}\right.$ & 90 & 80 & 70 & 60 & 50 & 40 & 30 & 20 & 10 & 0 & -10
\end{tabular} 


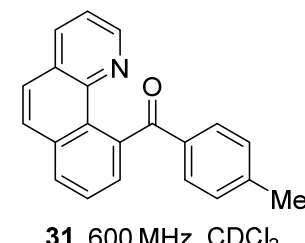

31, $600 \mathrm{MHz}, \mathrm{CDCl}_{3}$
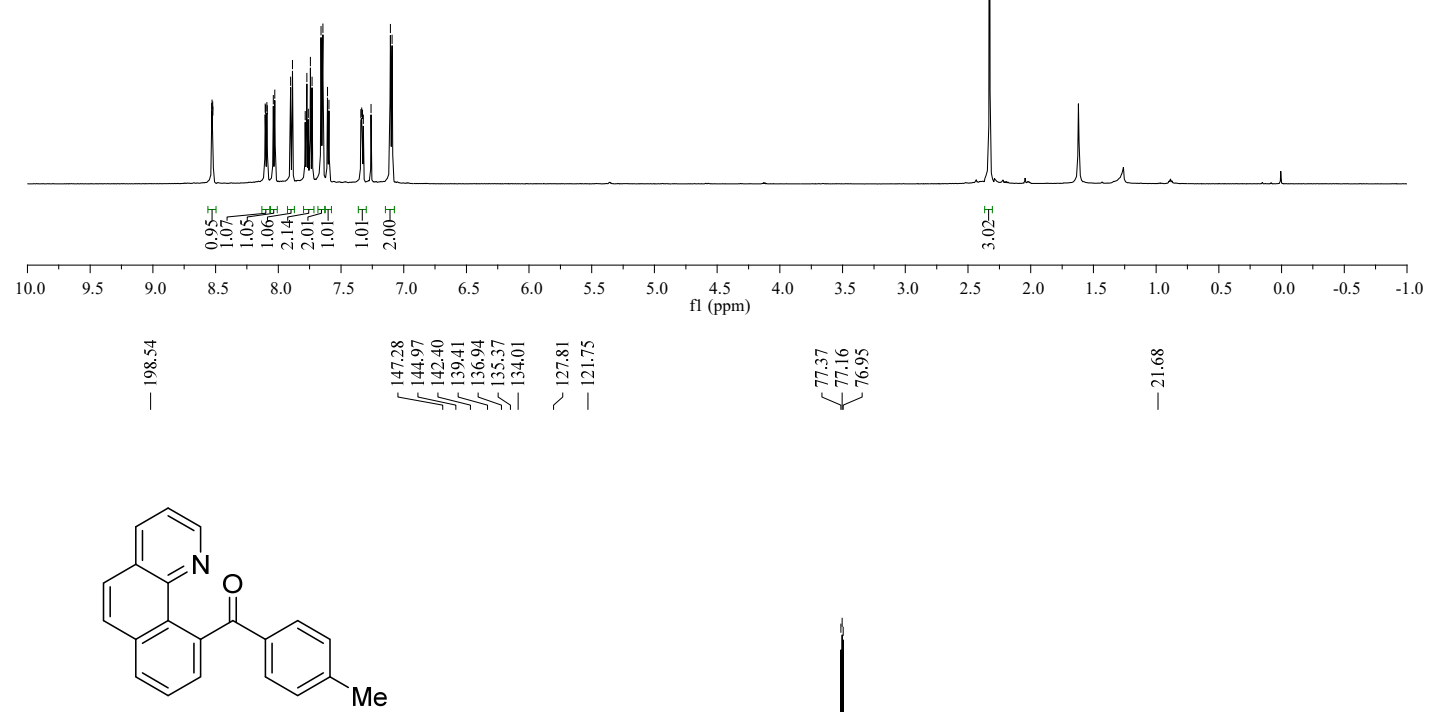

$31,150 \mathrm{MHz}, \mathrm{CDCl}_{3}$

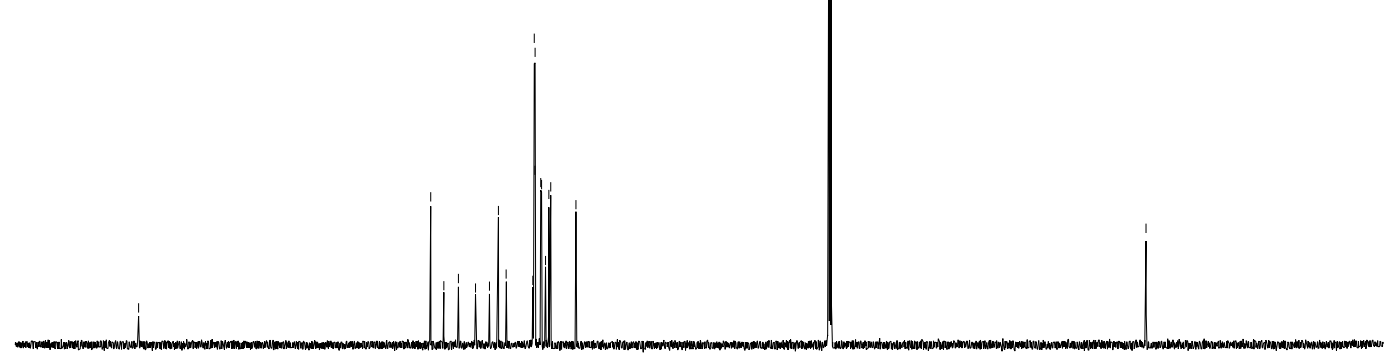

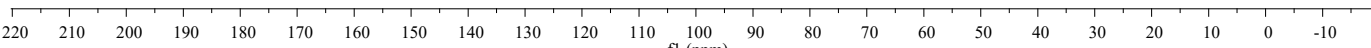




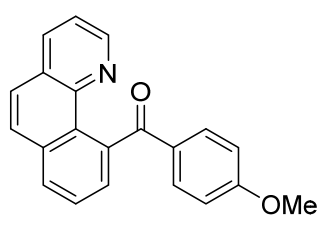

32, $600 \mathrm{MHz}, \mathrm{CDCl}_{3}$

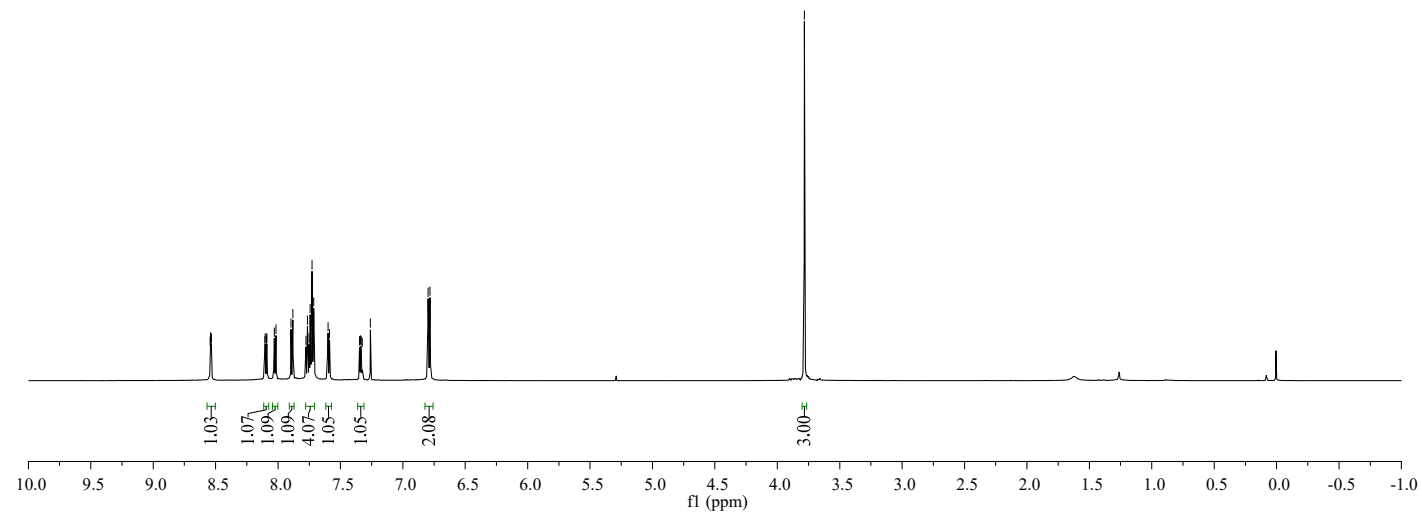

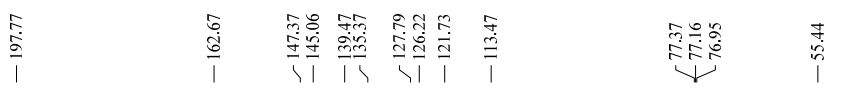

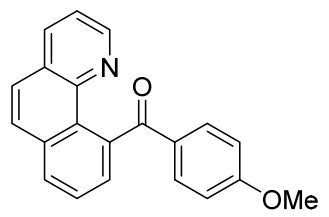

32, $150 \mathrm{MHz}, \mathrm{CDCl}_{3}$

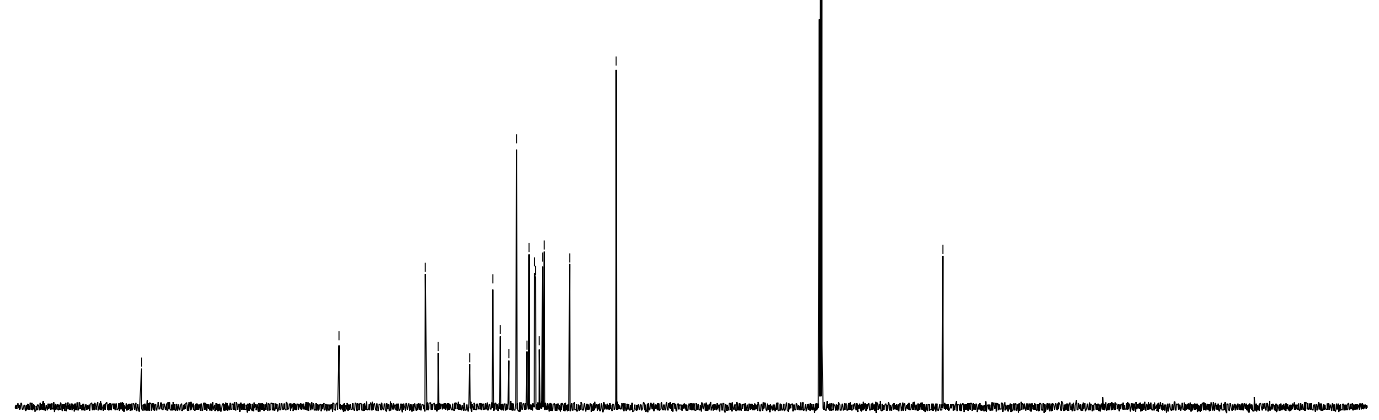

$\begin{array}{lllllllllllllllllllllllllll}220 & 210 & 200 & 190 & 180 & 170 & 160 & 150 & 140 & 130 & 120 & 110 & 100 & 90 & 80 & 70 & 60 & 50 & 40 & 30 & 20 & 10 & 0 & -10\end{array}$ 


\section{$\int_{1}^{1}$}

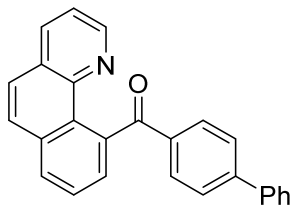

33, $600 \mathrm{MHz}, \mathrm{CDCl}_{3}$
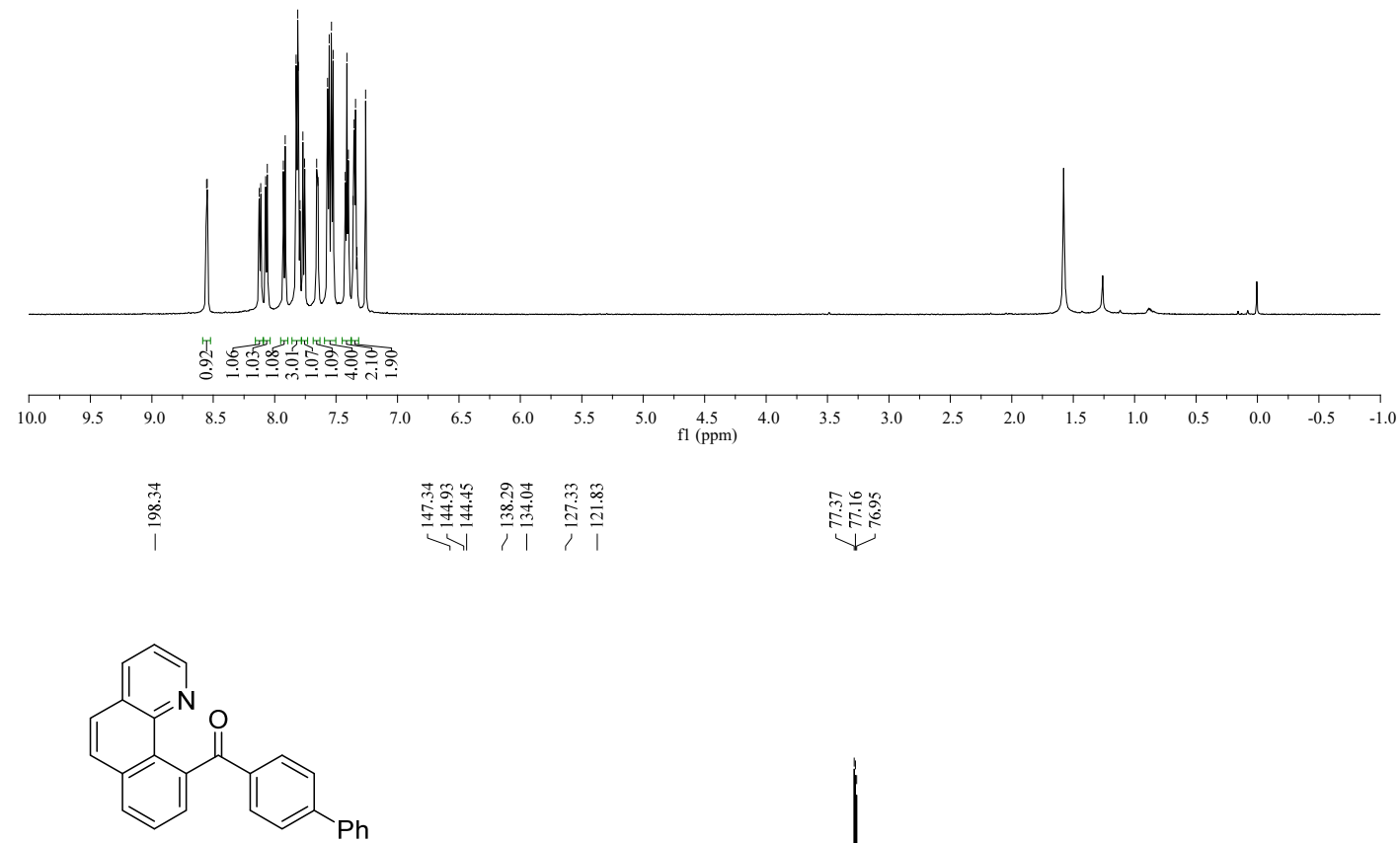

33, $150 \mathrm{MHz}, \mathrm{CDCl}_{3}$

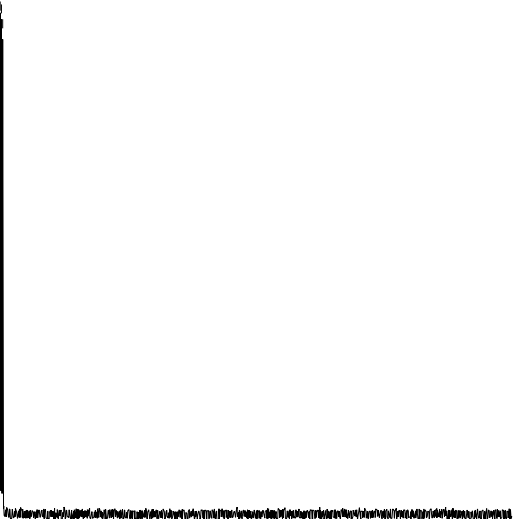

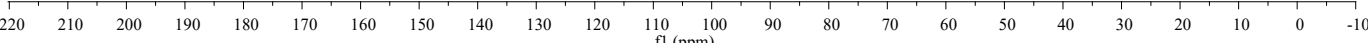




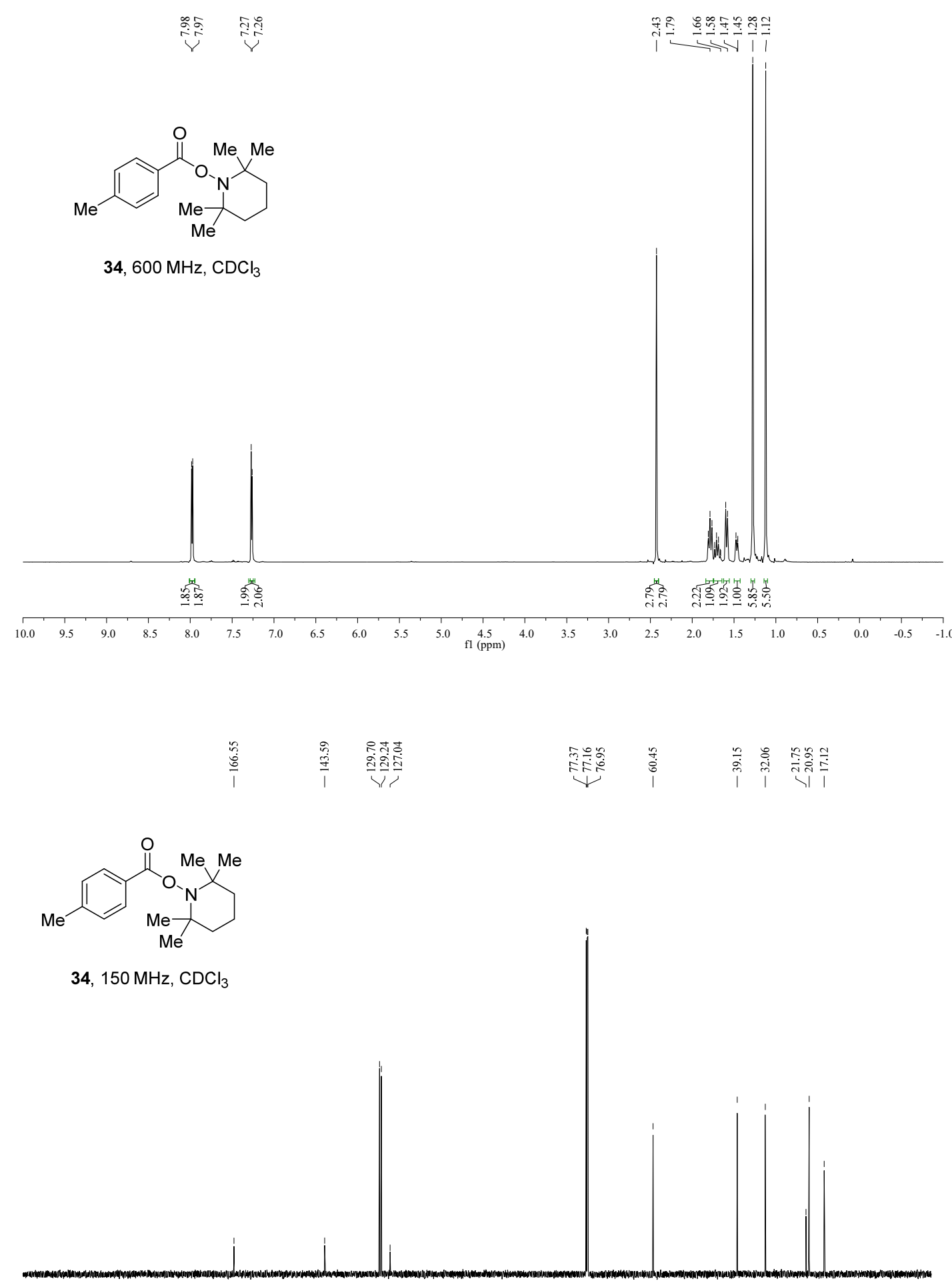

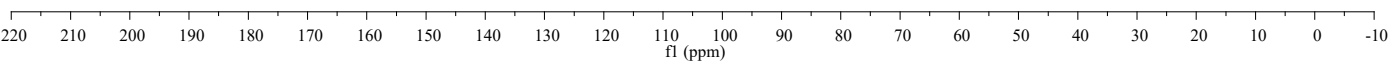

